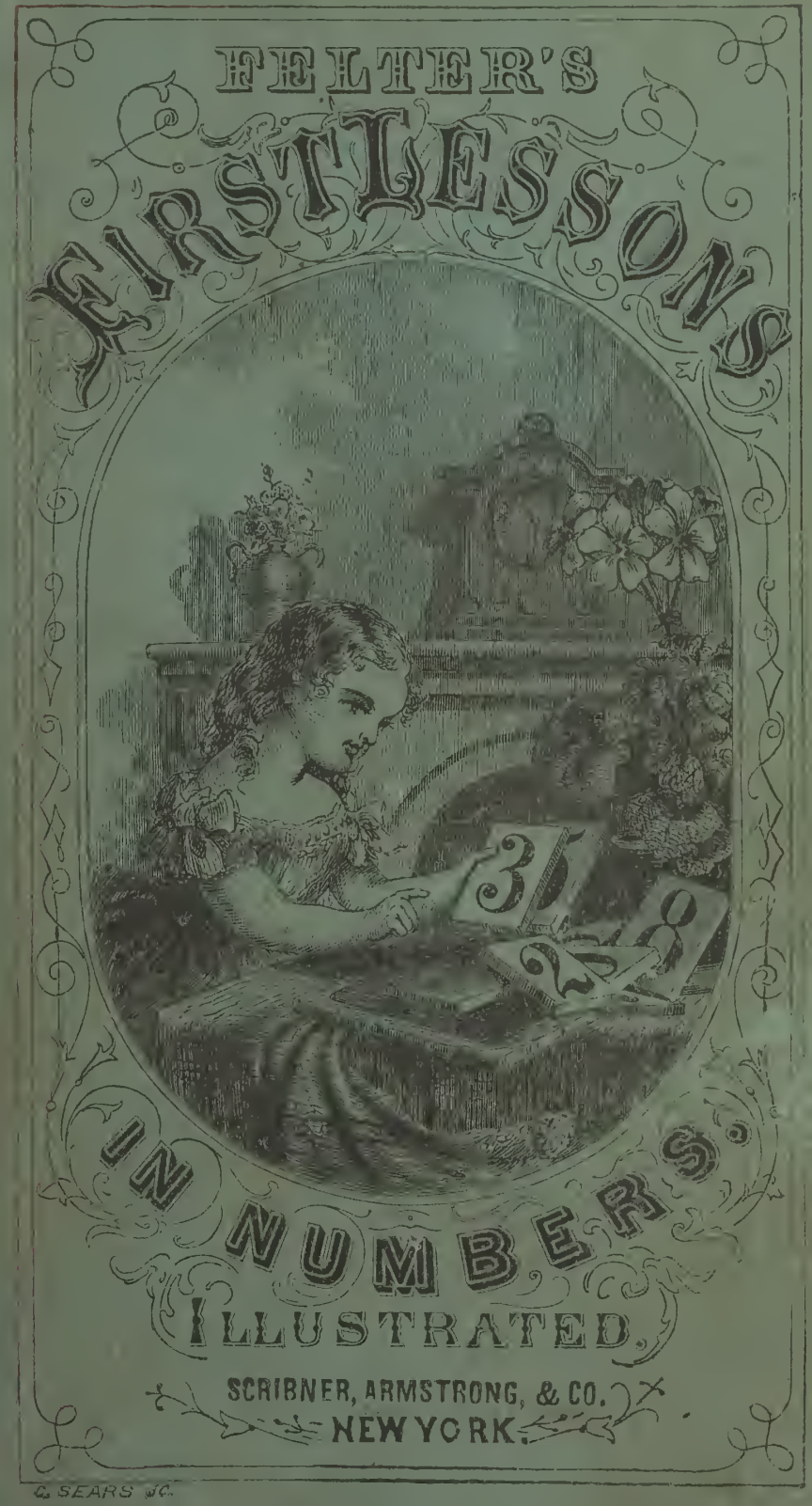



Feb"y a ny

Harch

A p p 1

Nil. $2 y$

6) Jube

Ja ly

Aciogut

Sep temíber

Qe - to: ber

No vemíner

De cern bet 
20

$\sigma$

r

2

r. 


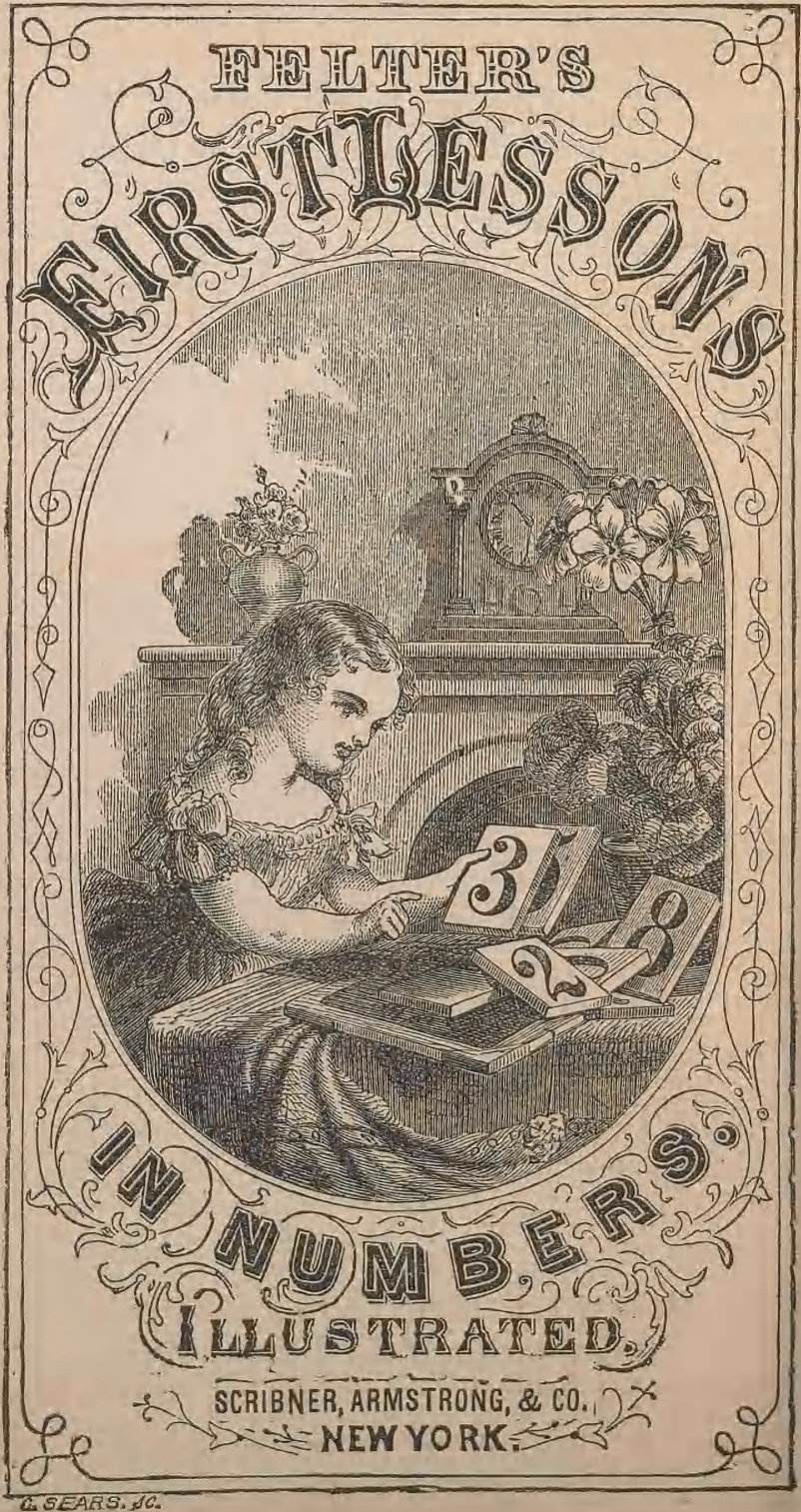




\section{NATURAL SERIES.}

THE

\section{FIRST LESSONS}

IN NUMBERS;

A.N

\section{ILLUSTRATED TABLE BOOK,}

DESIGNED FOR

\section{ELEMENTARY INSTRUCTION.}

BY

S. A. FELTER, A. M.,

LATE OF THE BROOKLYN COLLEGIATE AND POLYTECHNIO INSTTTUTE, AND AUTHOR of the "ARITHMetical aNALYSIS," ETC.

NEW YORK :

SCRIBNER, ARMSTRONG, AND CO.

HADLEY BROTHERS, CHICAGO, ILL. THOMPSON, BROWN, \& COMPANY, BOSTON, MAB. 
ENTRRED, according to Act of Congress, in the year 1865 , by S. A. FELTER,

In the Clerk' Office of the District Court of the United States for the Southera Diatrict of New York.

ENTERED, according to Aet of Congress, in the year 1868, by S. A . FELTER,

In the Clerk's Office of the District Court of the United States for the Southern District of Now York. 


\section{PRFF A CE.}

In the preparation of this little book, the author has aimed to make it simply a convenient storehouse from which the Teacher is to draw materials for the instruction of his pupils; and, therefore, it is not intended to " exempt the teacher from the labor of explaining orally, setting sums, \&c." It does not propose "to teach the pupil how to think," for this must be done by a living teacher; and no book, whatever its pretensions, can be more than an aid. In the first lessons, but little more should be done than to awaken the senses to the perception of numbers as exhibited in surrounding objects, and make the little pupils acquainted with some of the simpler facts. Whoever attempts to force upon them reasons, solutions, definitions, and relations, violates the fundamental law of the development of the human mind; and, although the pupils may become learned in the book, they are, nevertheless, on the broad road to conceited ignorance.

NEW YoRK, M M I, 1865. 


\section{TO THE TEACHER.}

THE teacher will readily perceive that this little book is emphatically an illustrated table-book, and not an elementary Arithmetic; and that its object is to suggest to the little pupil something TO Do. While it is impossible in a work so small to do more than give suggestions, the teacher will find little difficulty in supplying the deficiency by the use of the blackboard. Although there is nothing in the book which the children ought to be required to commit to memory as a set task, yet each step should be thoroughly mastered by means of oral and written exercises before proceeding to the next.

To accompany the series, there is a Manual prepared expressly for the use of the teacher; containing model lessons suggestive of the best methods of oral, written, individual, and class instruction.

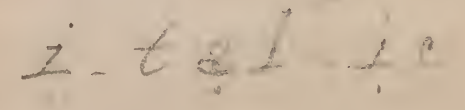


$0=1122$

$\therefore$

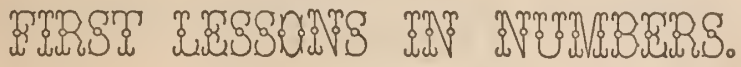

\section{LESSON I.}

Nore.-Each of the following Lessons is illustrated by a Model Exercise given in detail in a Teacher's Manual, prepared expressly to accompany this work.

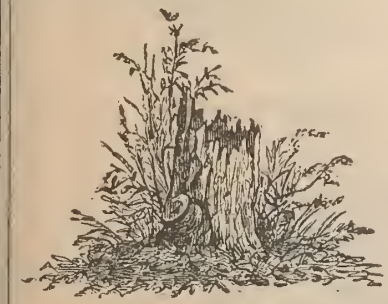

\section{One stump ; $1 ; 1$.}
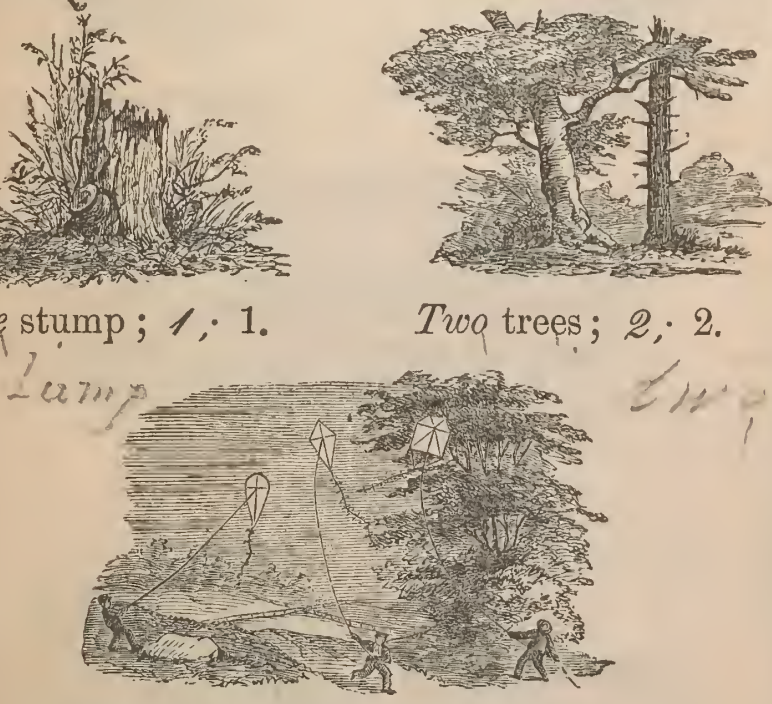

Three boys; Three kites ; 9.3.
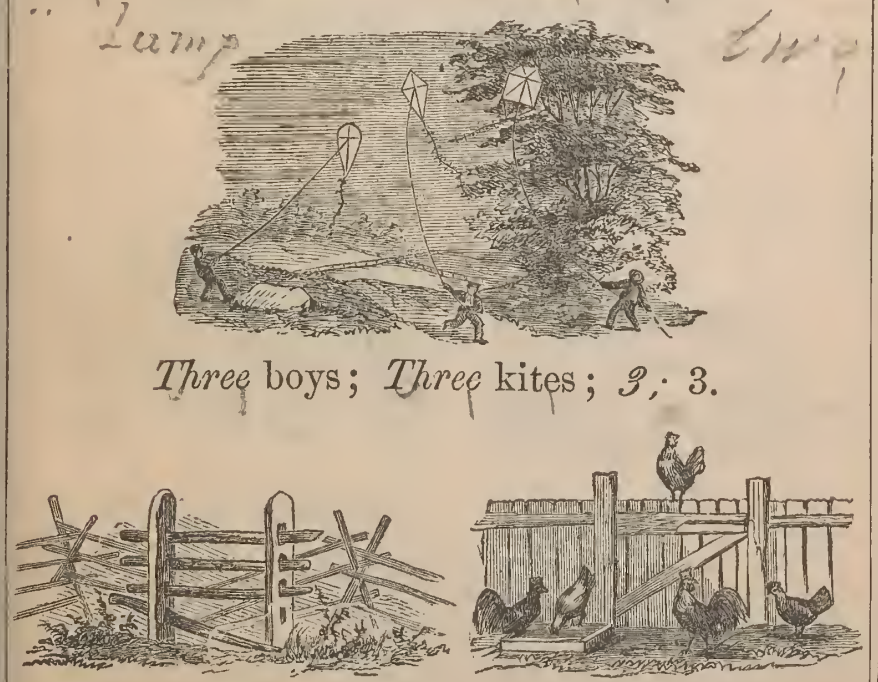

Four bars ; 4 ; 4.

Five fowls ; $5 ; 5$.

pozz I Eive




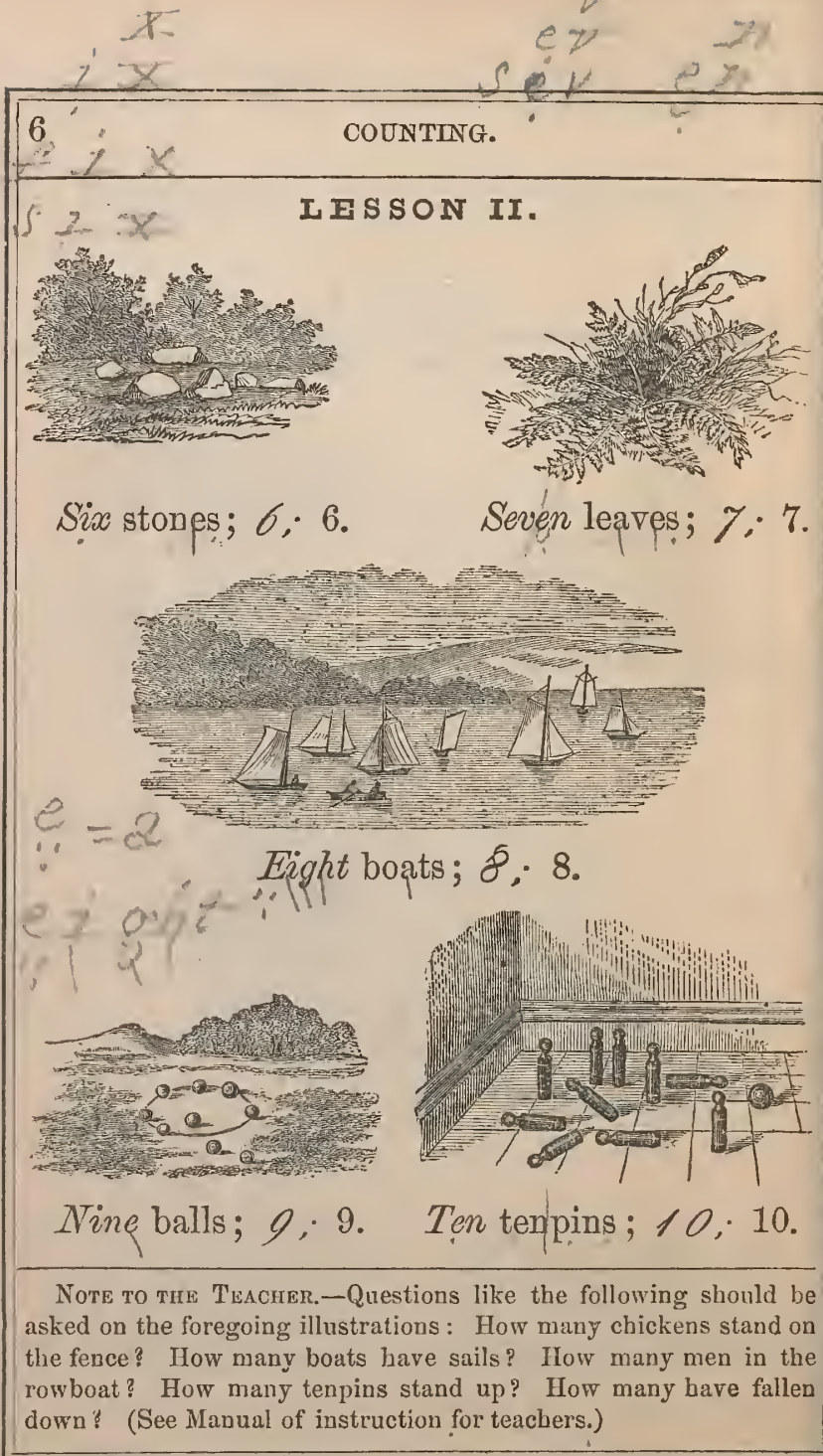




\section{IESSON III.*}

(See Manual, Sec. I., Exercise I.)

Copy and read the following exercises:

Ex. 1. (1) (2) (3) (4) (5) (6) (7) $\quad$ (8) $\quad$ (9) $\quad$ (10)

$\begin{array}{lllllllll} & 3 & 9 & 9 & 9 & 2 & 3 & 3 & 2\end{array}$

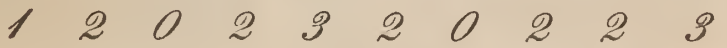

2191240 \& 2

$\begin{array}{lllllllllll}1 & 2 & 5 & 2 & 0 & 2 & 5 & \mathscr{Q} & 5 & 4\end{array}$

$\begin{array}{llllllllll}2 & 9 & 1 & 5 & 1 & 9 & 0 & 9 & 9 & 2\end{array}$

$\begin{array}{llllllllll}1 & 2 & 9 & 2 & 3 & 4 & 5 & 4 & 3\end{array}$

Ex. 2. (1) (2) (3) $\quad(4) \quad$ (5) $\quad$ (6) $\quad$ (7) $\quad$ (8) $\quad$ (9)

$7566 \% 76 \%$

$37979 \% 17 \%$

$60 \% 1798 \% 60$

$\begin{array}{lllllllllll}7 & 7 & 5 & 3 & 9 & 9 & 9 & 6 & 8 & 9\end{array}$

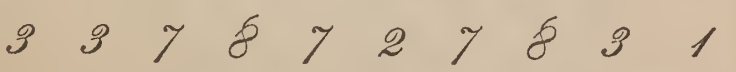

799160929

* Note for the Teacher.-These exercises should be copied neatly on the slate, and read at recitation by the members of the class. Particular attention should be given to the formation of figures. 


\section{IESSON IV.*}

(See Manual, Sec. I., Exercise I.)

$\mathscr{I}=1,1,1 . \quad\{\quad 3$ equals $1,1,1$, units. $5=1,1,1,1,1$. or 5 equals $1,1,1,1,1$ units. $\mathscr{Q}=1,1$. 2 equals 1,1 , units.

Copy and read the following exercises:

Ex. 1. Model.

(1) (2) (3)

(4) (5)

$\mathscr{P}=1,1,1$.

497 \& 5

3

$5=1,1,1,1,1.57 \% 6 \quad 6 \quad 7 \quad 7$

$\mathscr{\alpha}=1,1,1,1$.

$\begin{array}{llll}6 & 8 & 5 & 7\end{array}$

$\begin{array}{ll}6 & 6\end{array}$

Ex. 2

(1) (2)

(3)

(4)

(5)

(6)

(7)

(8) (9)

(10)

469

6

9

\&4 L

49

4

7917

41796

1

948

8

398

y 7

6

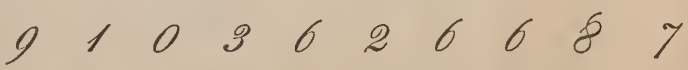

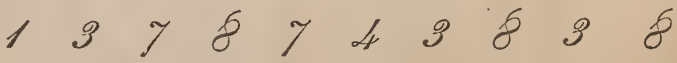

$\begin{array}{llllllll}7 & 8 & 7 & 9 & 7 & 7 & 9\end{array}$

* Note for the Teacher.-The teacher should expluin the meaning and use of the sign of equality ( $\Rightarrow$ ) in the following exercises. 

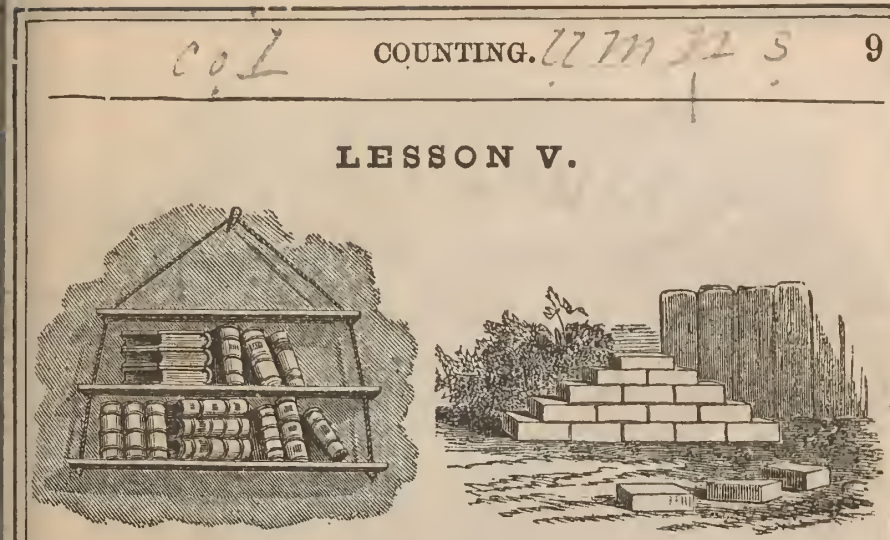

Fifteen books; $15 ; 15$. Eighteen bricks; 18 ; 18.

Ex. 1.

(1.)

(2.)

(3.)

(4.)

(5.)

(6.)

(7.)

(8.)

$1117 \quad 1316 \quad 15121019$

$\begin{array}{llllllllll}12 & 18 & 17 & 18 & 16 & 16 & 17 & 17\end{array}$

$\begin{array}{llllllllll}13 & 19 & 16 & 17 & 20 & 13 & 16 & 16\end{array}$

$142019 \quad 151817$ IL 19

$\begin{array}{llllllllll}1512 & 13 & 16 & 1 & 1 & 10 & 10 & 20\end{array}$

$\begin{array}{lllllllll}16 & 15 & 14 & 19 & 20 & 12 & 17 & 20\end{array}$

Questions.-How many books on the upper shelf? How many are lying down? How many bricks in the pile? How many bricks in the lowest row? How many bricks in the next row? How many are lying on the ground? How many books are standing up? How many books on the lower shelf? How many books on both shelves? If all the books were removed from the lower shelf, how many would remain on the upper shelf? If I should take away three books from the upper shelf, how many would remain ? How many would remain on both? etc. 


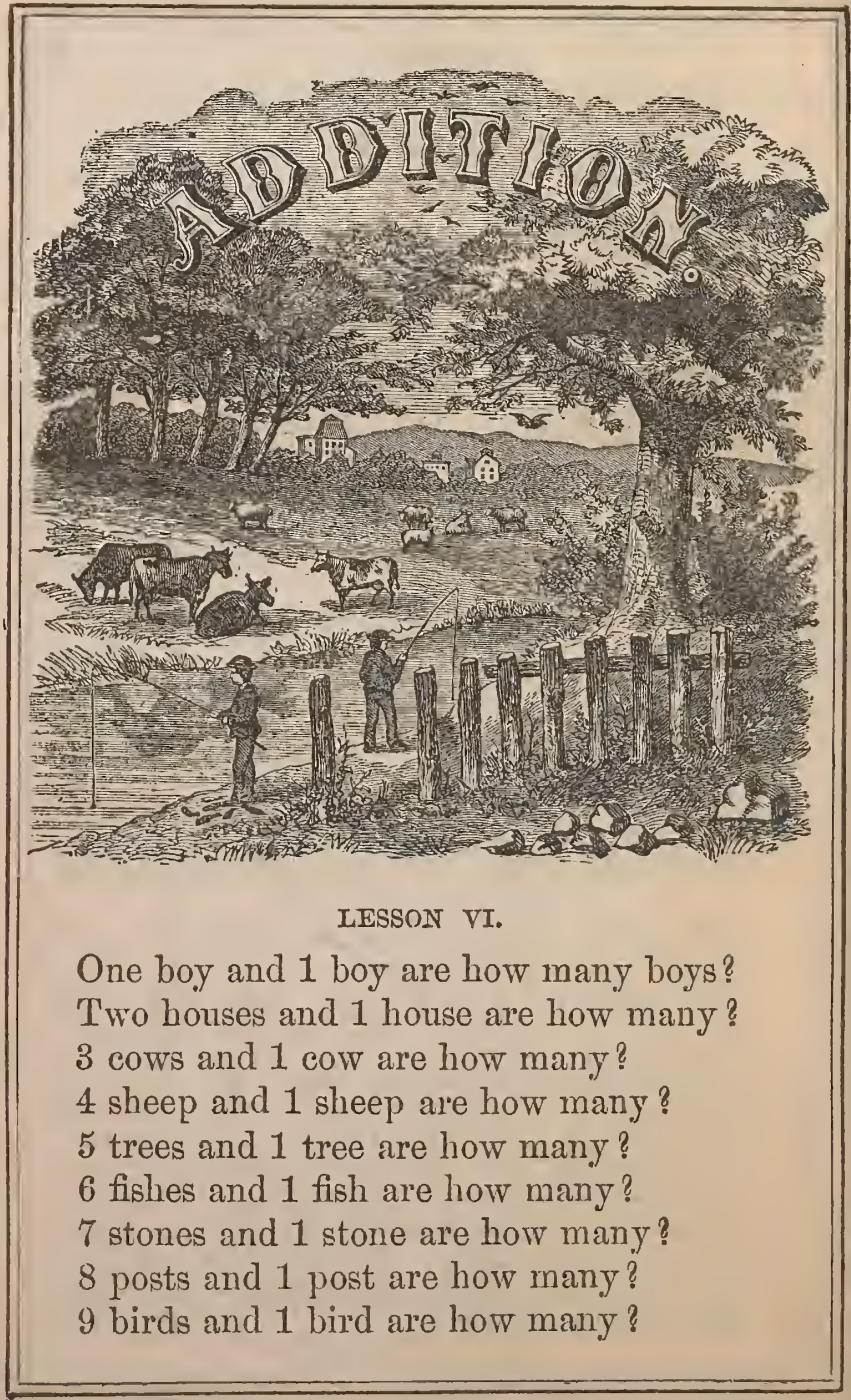




\section{IESSON VII.*}

(See Manual, Sec. I., Exercise II.)

Copy and complete the following tables:

Ex. 1. Model.

(1.)

(2)

(3.)

(4.)

$8+1=46+1 \quad \delta+18+1 \quad 4+1$

$4+1=5 \quad 1+6 \quad 9+1 \quad 3+12+1$

$7+1=8 \quad 3+1 \quad 7+1 \quad 1+9 \quad 3+1$

$2+1=32+1 \quad 1+76+16+1$

$4+1=5 \quad 3+16+1 \quad 8+j \quad 3+1$

$9+1=4 \quad 6+1 \quad 7+1 \quad 7+1 \quad 8+1$

Ex. 2 .

(1.)

(2.)

(3.)

(4.)

(5.)

$4+18+1 \quad 4+1 \quad 8+1 \quad 1+6$ $6+1 \quad 1+8 \quad \delta+1 \quad 7+1 \quad 8+1$ $\begin{array}{lllll}8+1 & 7+1 & 9+1 & 3+1 & 1+8\end{array}$ $\begin{array}{lllllll}1+6 & 6+1 & 6+1 & 9+1 & 1+7\end{array}$ $7+19+1 \quad 3+1 \quad 1+9 \quad 1+7$ $6+13+1 \quad 4+1 \quad 1+7 \quad 1+7$

* Note for the Tencher-Before copying the following lessons the sigu of addition $(+)$ should be explained to the pupils. 


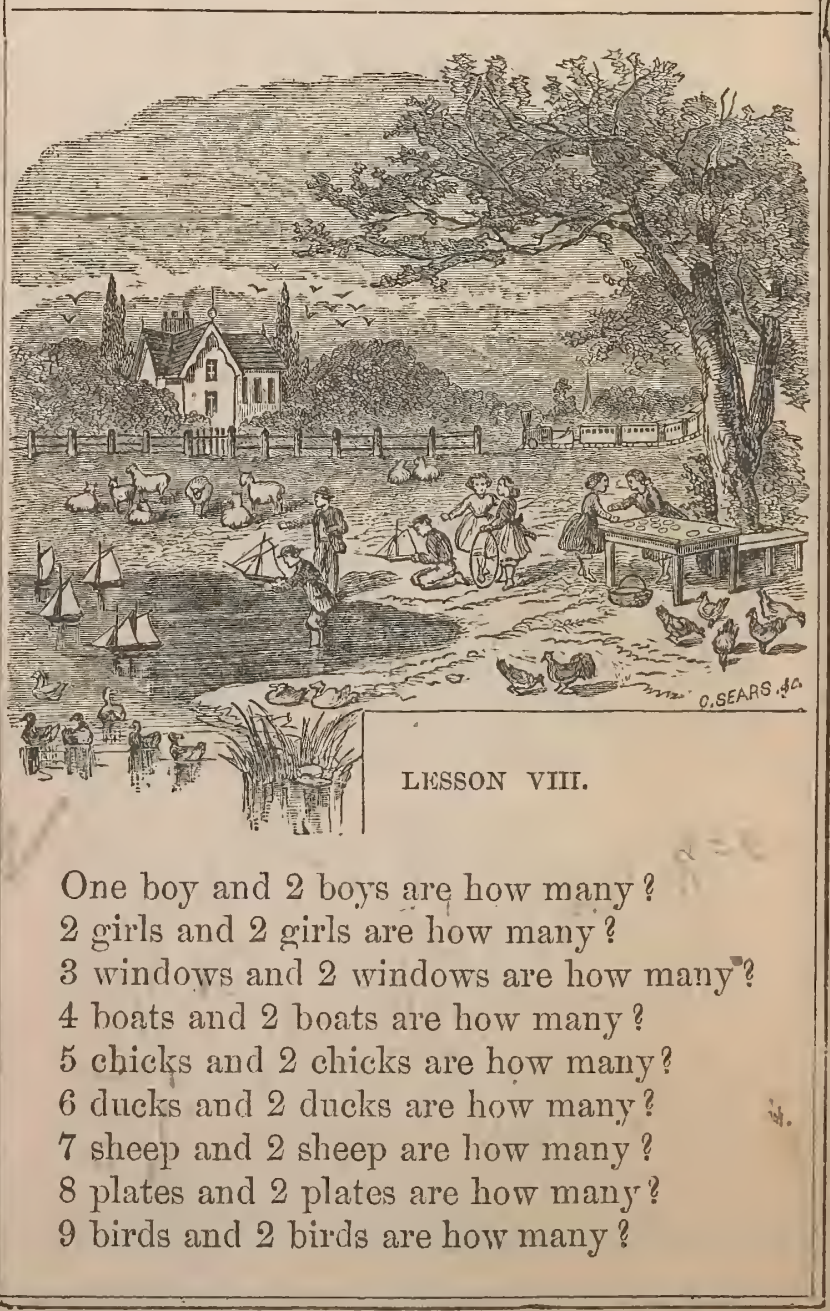




\section{IESSON IX.}

(See Manual, Sec. I., Exercise II.)

Copy and complete the following tables:

Ex. 1.

(2.)

(3.)

(4.)

(5.)

(6.)

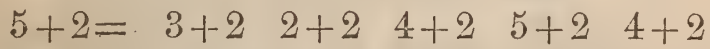

$4+2=2+3 \quad 2+2 \quad 2+5 \quad 2+5 \quad 2+2$

$2+4=\begin{array}{lllll}4+2 & 3+2 & 4+2 & 3+2 & 1+2\end{array}$

$5+2=2+5 \quad 2+2 \quad 1+2 \quad 2+3 \quad 2+1$

$2+2=2+4 \quad 2+2 \quad 2+3 \quad 1+2 \quad 3+2$

$3+2=\begin{array}{lllll}3+2 & 1+2 & 1+2 & 2+1 & 1+2\end{array}$

Ex. 2.

(1.)

(2.)

(3.)

(4.)

(5.)

(6.)

$2+1 \quad 1+4 \quad 2+3 \quad 4+2 \quad 5+1 \quad 7+1$ $\begin{array}{llllll}1+2 & 3+2,3+2 & 5+2 & 4+1 & 4+2\end{array}$ $\begin{array}{lllll}1+3 & 2+3-1+5 & 6+1 & 2+4 & 1+2\end{array}$ $\begin{array}{llllll}2+2 & 4+1 & 5+2 & 1+6 & 5+2 & 0+2\end{array}$ $\begin{array}{llllll}4+1 & 5+1 & 2+5 & 2+5 & 4+2 & 2+0\end{array}$ $\begin{array}{llllll}3+2 & 1+5 & 3+2 & 5+1 & 2+5 & 4+2\end{array}$

Ex. 3. (1.) (2.) (3.) (4.) (5.) (6.) $\begin{array}{llllll}6+2 & 8+2 & 3+2 & 5+2 & 2+7 & 4+2\end{array}$ $\begin{array}{llllll}2+6 & 7+2 & 5+2 & 2+3 & 0+7 & 2+2\end{array}$ $\begin{array}{llllll}7+2 & 6+2 & 2+5 & 2+7 & 0+2 & 5+2\end{array}$ $\begin{array}{llllll}2+7 & 8+2 & 7+2 & 2+9 & 2+0 & 7+2\end{array}$ $\begin{array}{llllll}4+2 & 1+2 & 2+8 & 2+8 & 1+0 & 6+2\end{array}$ $\begin{array}{llllll}7+2 & b+2 & 2+7 & 2+6 & 8+0 & 8+2\end{array}$ 


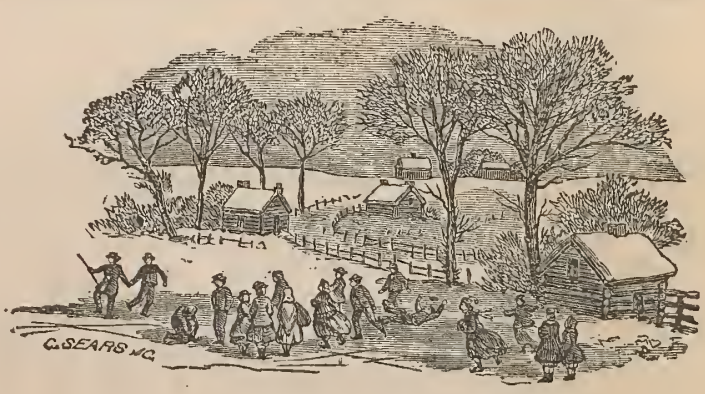

LESSON $X$.

One boy and 3 boys are how many?

2 houses and 3 houses are how many?

3 trees and 3 trees are how many?

4 girls and 3 girls are how many?

5 caps and 3 caps are how many?

6 skates and 3 skates are how many?

7 posts and 3 posts are how many?

8 sleds and 3 sleds are how many?

9 hoods and 3 hoods are how many?

Nore.-The teacher should dictate problems referring to the objects in the illustrations, thus: Two boys have hold of hands; how many would there be if another should join them? There are six trees; if two were cut down, how many would remain standing? Two little girls areskating and two are looking on ; how many in all? There are five houses; if two should be burned up how many would remain? If one boy has two skates, how many skates will two boys have? There are eight boys skating, one of them has fallen down; how many remain standing? One house and one house are how many? One tree and five trees are how many? Four trees and two trees are how many? Two trees and four trees are how many? 


\section{LESSON XI.}

(See Manual, Sec. I., Exercise II.)

Copy, complete, and read the following tables:

Ex. 1.
(1.)
(2.)
(3.)
(4.)
(5.)

(6.)

$\begin{array}{llllll}1+3 & 3+1 & 4+3 & 1+5 & 3+4 & 4+3\end{array}$ $\begin{array}{llllll}3+1 & 3+4 & 3+2 & 1+3 & 2+4 & 3+2\end{array}$ $\begin{array}{llllll}3+4 & 1+4 & 2+1 & 1+6 & 4+3 & 2+1\end{array}$ $\begin{array}{llllll}6+1 & 3+1 & 1+3 & 2+4 & 4+2 & 3+5\end{array}$ $\begin{array}{llllll}5+2 & 5+2 & 4+3 & 2+5 & 5+2 & 5+2\end{array}$ $\begin{array}{llllll}4+3 & 2+5 & 5+1 & 3+2 & 5+3 & 3+2\end{array}$

Ex. 2.

(1.)

(2.)

(3.)

(4.)

(5.)

(6.)

$\begin{array}{llllll}5+3 & 4+3 & 3+9 & 4+3 & 7+3 & 6+3\end{array}$

$\begin{array}{llllll}3+5 & 5+3 & 7+3 & 7+3 & 4+3 & 7+3\end{array}$

$\begin{array}{llllll}6+1 & 7+3 & 6+3 & 3+7 & 3+4 & 3+7\end{array}$

$\begin{array}{llllll}6+3 & 8+3 & 3+9 & 4+3 & 7+3 & 5+3\end{array}$

$\begin{array}{llllll}5+3 & 6+3 & 7+3 & 6+3 & 8+3 & 4+3\end{array}$

$\begin{array}{llllll}6+3 & 9+3 & 6+3 & 3+6 & 3+4 & 3+0\end{array}$

Ex. 3. (1.)

(2.)

(3.)

(4.)

(5.)

(6.)

$\begin{array}{llllll}6+3 & 3+3 & 3+7 & 6+3 & 2+6 & 3+3\end{array}$ $\begin{array}{llllll}3+6 & 7+3 & 2+6 & 3+2 & 6+1 & 2+3\end{array}$ $\begin{array}{llllll}9+3 & 2+3 & 3+2 & 2+8 & 1+8 & 4+3\end{array}$ $\begin{array}{llllll}8+3 & 2+7 & 1+5 & 8+3 & 8+3 & 3+2\end{array}$ $\begin{array}{lllllll}3+8 & 6+2 & 1+7 & 9+3 & 3+4 & 2+9\end{array}$ $\begin{array}{llllll}6+2 & 2+3 & 6+1 & 4+2 & 7+2 & 3+2\end{array}$ 


\section{ADDITION.}

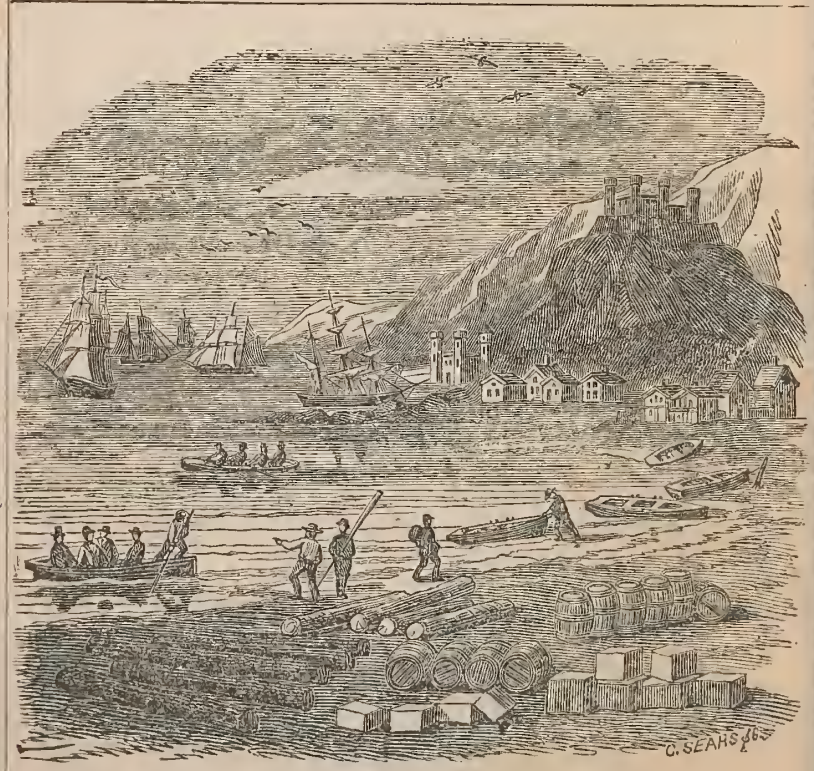

LESSON XII.

One ship and 4 ships are how many?

2 boats and 4 boats are how many?

3 towers and 4 towers are low many?

4 honses and 4 houses are how many?

5 logs and 4 logs are how many?

6 barrels and 4 barrels are how many?

7 sea-gulls and 4 sea-gulls are how many?

8 masts and 4 masts are how many?

9 men and 4 men are how many? 


\section{IESSON XIII.}

(See Manual, Sec. I., Exercise 1I.)

Copy, complete, and read the following tables:

Ex. 1 .

(1.).

(2.)

(3.)

(4.)

(5.)

(6.)

$\begin{array}{llllll}4+2 & 3+4 & 5+4 & 0+4 & 1+3 & 7+1\end{array}$

$2+4 \quad 2+4 \quad 4+2 \quad 4+0 \quad 4+2 \quad 6+3$

$\begin{array}{llllll}3+2 & 2+3 & 2+3 & 7+1 & 2+3 & 4+2\end{array}$

$2+1 \quad 3+1 \quad 3+5 \quad 8+0 \quad 3+1 \quad 7+3$

$\begin{array}{llllll}3+4 & 4+2 & 5+2 & 8+1 & 8+1 & 6+2\end{array}$

$\begin{array}{llllll}4+3 & 4+4 & 2+4 & 3+1 & 1+8 & 2+3\end{array}$

Ex. 2. (1.) (2.) (3.) (4.) (5.) (6.)

$\begin{array}{llllll}5+4 & 1+6 & 7+4 & 2+4 & 4+0 & 6+4\end{array}$ $\begin{array}{llllll}6+4 & 4+7 & 3+4 & 0+4 & 3+4 & 4+6\end{array}$ $\begin{array}{llllll}4+6 & 4+3 & 8+4 & 4+0 & 0+4 & 6+2\end{array}$ $\begin{array}{llllll}3+6 & 4+8 & 7+4 & 8+4 & 9+4 & 6+4\end{array}$ $\begin{array}{llllll}4+5 & 4+9 & 6+4 & 9+4 & 7+4 & 4+6\end{array}$ $\begin{array}{llllll}5+6 & 4+7 & 3+4 & 3+4 & 4+7 & 3+4\end{array}$

12x, 2. (1.)

(4.)

(5.)

(6.)

$6+4 \quad 4+2 \quad 5+4-9+3 \quad 0+4 \quad 4+1$ $\begin{array}{llllll}4+6 & 2+4 & 3+4 & 4+3 & 0+3 & 1+0\end{array}$ $3+4-6+4 \quad 2+4 \quad 2+2 \quad 3+2 \quad 0+1$ $\begin{array}{llllll}2+4 & 8+2 & 7+3 & 3+3 & 2+0 & 1+1\end{array}$ $\begin{array}{llllll}4+3 & 2+4 & 6+3 & 4+4 & 1+0 & 2+2\end{array}$ $\begin{array}{llllll}6+3 & 6+4 & 8+2 & 7+3 & 3+2 & 3+3\end{array}$ 


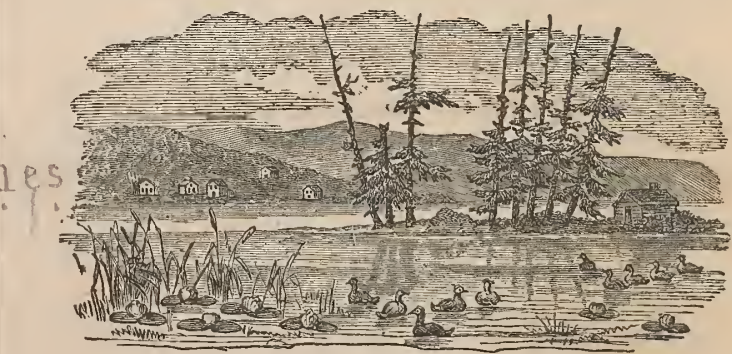

LESSON XIV.

One house and 5 houses are how many?

2 lilies and 5 lilies are how many?

3 pines and 5 pines are how many?

4 windows and 5 windows are how many?

5 ducks and 5 ducks are how many?

6 islands and 5 islands are 11 islands.

7 leaves and 5 leaves are 12 leaves.

8 flowers and 5 flowers are 13 flowers.

9 doors and 5 doors are 14 doors.

To тhe Teacher.-Oral questions and problems referring to the above and following illustrations should be given to the class. (See Manual Ex. III. Less. II.) The pupils should be required to ask questions of each other referring to the objects in the illustrations.

Problems.-If there are six houses and each house has one door, how many doors have all the houses? Five of the doors are standing open, how many are shut? If a hunter should shoot five of the ducks in the pond, how many would escape? If a little girl should pick two of the water lilies, how many would remain? Five ducks and 3 ducks are how many? Three trees and 5 trees are how many? Five houses and two houses are how many? Five men and two men are how many? 


\section{LESSON X V .}

(See Manual, Sec. I., Exercise II.)

Copy, complete, and read the following tables:

Ex. 1.
(1.)
(2.)
(3.)
(4.)
(5.)

(6.)

$\begin{array}{llllll}5+6 & 6+5 & 6+5 & 5+6 & 6+7 & 3+5\end{array}$

$\begin{array}{llllll}5+3 & 7+5 & 5+6 & 7+5 & 7+5 & 6+5\end{array}$

$\begin{array}{llllll}5+7 & 8+5 & 7+5 & 8+5 & 3+5 & 5+6\end{array}$

$\begin{array}{llllll}5+8 & 9+5 & 5+8 & 5+6 & 6+5 & 7+5\end{array}$

$\begin{array}{llllll}5+9 & 8+5 & 9+5 & 5+7 & 7+5 & 3+5\end{array}$

$\begin{array}{llllll}5+6 & 3+5 & 7+5 & 5+6 & 5+6 & 6+5\end{array}$

Ex. 2. (1.) (2.) (3.) (4.) (5.) (6.)

$\begin{array}{llllll}3+5 & 5+2 & 5+3 & 5+2 & 6+4 & 4+3\end{array}$ $\begin{array}{llllll}5+2 & 6+2 & 5+2 & 8+1 & 5+4 & 7+2\end{array}$ $\begin{array}{llllll}2+3 & 7+2 & 3+5 & 7+2 & 7+3 & 4+3\end{array}$ $\begin{array}{llllll}3+5 & 5+3 & 9+1 & 7+3 & 6+4 & 4+4\end{array}$ $\begin{array}{llllll}4+2 & 5+5 & 8+2 & 3+2 & 5+5 & 6+2\end{array}$ $\begin{array}{llllll}4+5 & 3+5 & 7 .+3 & 4+3 & 9+1 & 6+2\end{array}$
12x. 3. (1.)
(2.)
(3.)
(4.)
(5.)
(6.)

$7+4 \quad 4+6 \quad 7+5 \quad 9+3 \quad 4+6 \quad 4+3$ $\begin{array}{llllll}4+5 & 6+4 & 5+6 & 8+3 & 3+2 & 6+3\end{array}$ $\begin{array}{llllll}6+4 & 3+5 & 7+2 & 7+5 & 3+1 & 5+2\end{array}$ $\begin{array}{llllll}8+5 & 5+5 & 9+3 & 6+2 & 6+7 & 4+7\end{array}$ $7+6 \quad 2+2 \quad 4+3 \quad 9+3 \quad 6+8 \quad 4+4$ $\begin{array}{llllll}7+3 & 3+3 & 9+1 & 7+2 & 3+7 & 5+5\end{array}$ 


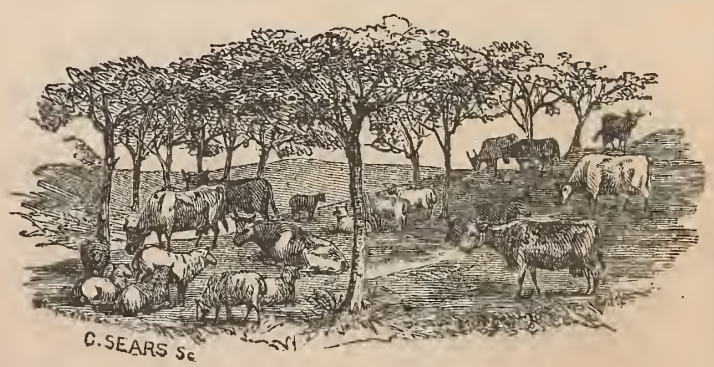

LESSON XVI.

One branch and 6 branches are 7 branches. 2 stones and 6 stones are 8 stones.

3 cows and 6 cows are 9 cows. 4 sheep and 6 sheep are 10 sheep. 5 trees and 6 trees are 11 trees.' 6 lambs and 6 lambs are 12 lambs. 7 feet and 6 feet are 13 feet. 8 men and 6 inen are 14 men. 9 birds and 6 birds are 15 birds.

Problems. - If 3 cows were driven home, how many would remain in the pasture? If a boy should drive away 6 of the sheep, how many would remain? Since 1 cow has 4 feet, how many feet have 2 cows? A farmer had 10 sheep, he sold 6 of them, how many had he left? There are 9 cows in the pasture, 6 of the cows have been milked; how many have yet to be milked? Two sheep aud 3 sheep are how many? Two cows and 4 cows are how many? Five trees and 4 trees are how many? How many feet have 2 sheep? How many feet have 4 sheep? How many feet have 8 sheep? How many horns have three cows? How many horns have 5 cows? How many borns have 6 cows? Five boats and 3 boats are how many? Six boys and 5 boys are how many? 


\section{LESSON XVII.}

(See Manual, Sec. I., Exercise II.)

Copy, complete, and read the following tables:

Ex. 1.

(2)

(3.)

(4.)

(5.)

$\begin{array}{llllll}8+3 & 3+3 & 8+3 & 6+2 & 8+2 & 8+2\end{array}$

$\begin{array}{llllll}7+2 & 6+5 & 7+4 & 8+3 & 7+3 & 7+2\end{array}$

$\begin{array}{llllll}9+1 & 2+2 & 6+2 & 7+4 & 2+4 & 3+6\end{array}$

$\begin{array}{llllll}8+3 & 1+1 & 9+1 & 5+4 & 9+2 & 7+2\end{array}$

$\begin{array}{llllll}-7+3 & 7+3 & 7+4 & 4+4 & 6+1 & 4+2\end{array}$

$\begin{array}{llllll}2+3 & 6+2 & 3+2 & 2+2 & 3+0 & 4+1\end{array}$

Ex. 2.

(1.)

(2.)

(3.) (4.)

(5.)

(6.)

$\begin{array}{llllll}6+5 & 4+7 & 4+6 & 6+9 & 8+6 & 4+6\end{array}$ $\begin{array}{llllll}6+6 & 6+6 & 6+7 & 9+6 & 3+6 & 6+8\end{array}$ $\begin{array}{llllll}3+6 & 3+6 & 6+8 & 8+6 & 6+7 & 6+9\end{array}$ $\begin{array}{llllll}4+6 & 8+6 & 6+3 & 7+6 & 7+6 & 6+3\end{array}$ $\begin{array}{llllll}9+6 & 6+8 & 6+9 & 4+6 & 9+6 & 6+4\end{array}$ $\begin{array}{llllll}7+6 & 3+8 & 6+7 & 3+6 & 8+6 & 6+8\end{array}$

Fix. 3 . (1.)

(4.)

(5.)

(6.)

$\begin{array}{llllll}8+6 & 3+3 & 8+3 & 9+3 & 4+7 & 4+6\end{array}$ $\begin{array}{llllll}6+7 & 4+4 & 5+7 & 6+7 & 6+3 & 3+7\end{array}$ $\begin{array}{llllll}3+4 & 5+5 & 6+9 & 8+6 & 8+3 & 8+3\end{array}$ $\begin{array}{llllll}6+3 & 6+6 & 3+7 & 3+7 & 4+7 & 4+3\end{array}$ $\begin{array}{llllll}4+4 & 2+2 & 8+6 & 4+3 & 6+7 & 7+2\end{array}$ $\begin{array}{llllll}3+3 & 0+0 & 4+7 & 7+2 & 4+7 & 6+3\end{array}$ 


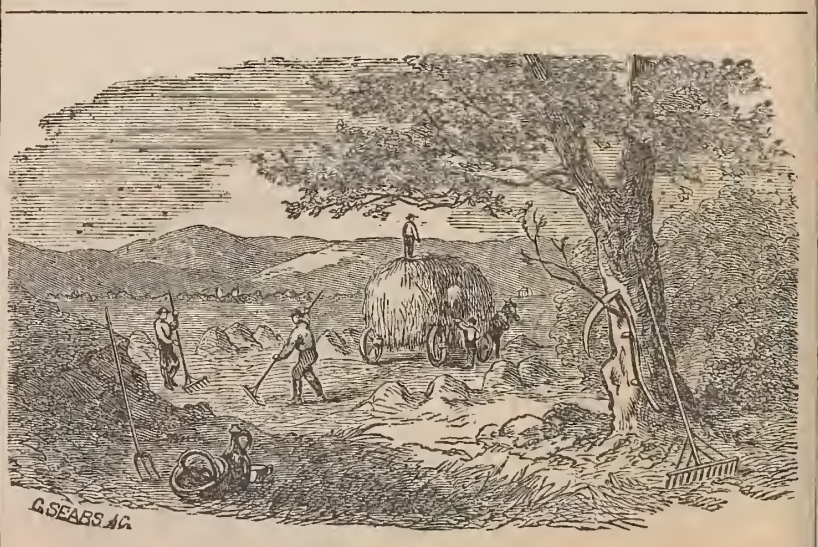

- LESSON XVIII.

One rake and 7 rakes are 8 rakes. 2 forks and 7 forks are 9 forks. 3 horses and 7 horses are 10 horses. 4 men and 7 men are 11 men. 5 scythes and 7 scythes are 12 scythes. 6 jugs and 7 jugs are 13 jugs. 7 cups and 7 cups are 14 cups. 8 wagons and 7 wagons are 15 wagons. 9 trees and 7 trees are 16 trees.

Prob.-There are five bunches of hay on one side of the wagon and five on the other, how many on both? There are three rakes, one is standing against a tree, the rest are in use; how many are in use? Four rakes and 5 rakes are how many? Five baskets and 6 baskets are how many? Six cups and 5 cups are how many? Eight horses and 3 horses are how many? Seven trees and 4 trees are how many? Six loads of hay and 2 loads are how many? 


\section{LES SON XIX.}

(See Manual, Sec. I., Exercise II.)

Copy, complete, and read the following tables:
Ex. 1.
(2.)
(3.)
(4.)
(5.)
(6.)

c. $3+7 \quad 3+4 \quad 4+4 \quad 3+1 \quad 4+3 \quad 8+3$ $\begin{array}{llllll}4+3 & 9+3 & 3+3 & 7+2 & 6+4 & 4+2\end{array}$ $\begin{array}{llllll}6+4 & 9+2 & 2+2 & 7+4 & 8+4 & 9+3\end{array}$ $\begin{array}{llllll}3+4 & 7+4 & 1+1 & 7+3 & 5+3 & 7+2\end{array}$ $\begin{array}{llllll}8+4 & 8+3 & 0+0 & 6+3 & 7+4 & 3+8\end{array}$ $\begin{array}{llllll}6+4 & 9+3 & 0+1 & 7+2 & 9+3 & 6+2\end{array}$

Ex. 2. (1.)
(2.)
(3.)
(4.)

(5.)

(6.)

$\begin{array}{llllll}7+4 & 7+8 & 7+6 & 9+7 & 7+3 & 8+7\end{array}$

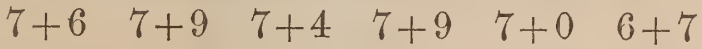

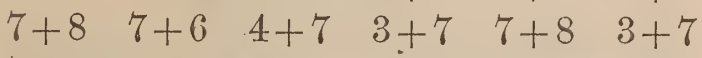
$\begin{array}{llllll}7+2 & 7+2 & 6+7 & 6+7 & 7+9 & 7+3\end{array}$ $\begin{array}{llllll}7+3 & 7+3 & 8+7 & 4+7 & 6+7 & 7+8\end{array}$ $\begin{array}{llllll}7+2 & 7+0 & 3+7 & 4+7 & 3+7 & 7+3\end{array}$

Ex. 3. (1.)

(2.) (3.)

(4.) (5.)

(6.)

$\begin{array}{llllll}7+8 & 4+3 & 4+6 & 9+7 & 6+7 & 4+6\end{array}$ $\begin{array}{llllll}6+7 & 6+7 & 6+7 & 4+7 & 6+8 & 7+8\end{array}$ $\begin{array}{llllll}3+8 & 3+8 & 8+7 & 6+3 & 3+4 & 3+4\end{array}$ $\begin{array}{llllll}9+7 & 4+7 & 3+8 & 8+3 & 7+2 & 6+7\end{array}$

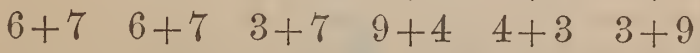
$\begin{array}{llllll}4+7 & 3+7 & 4+3 & 7+8 & 6+7 & 4+7\end{array}$ 


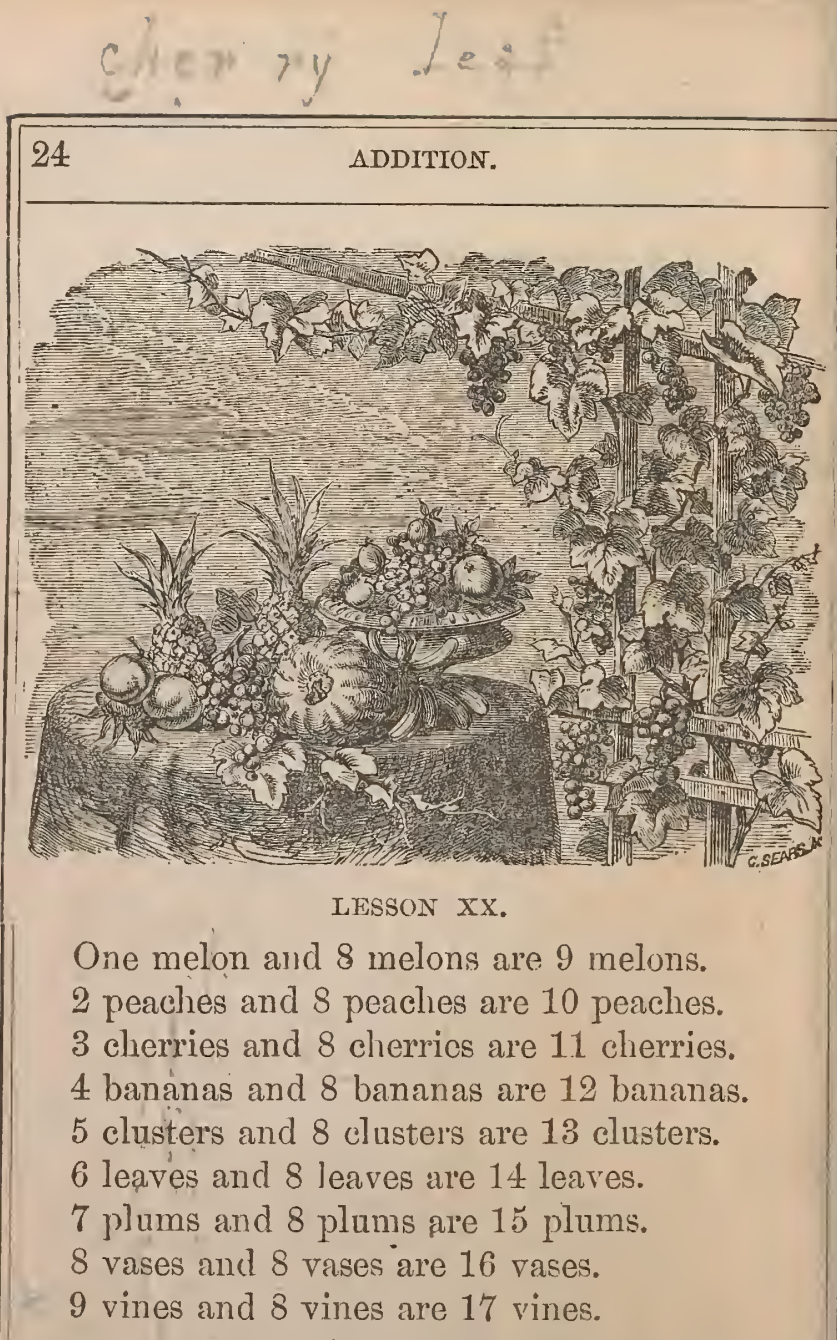

Problams.-Two peaches and 5 peaches are how many? Four pine-apples and 6 pine-apples are how many? Eight oranges and 4 oranges are how many? 


\section{IESSON XXI.}

(See Manual, Sec. I., Exercise II.)

Copy, complete, and read the following tables:
Ex. 1.
(1.)
(2.)
(3)
(4.)
(5.)
(6.)

$8 \begin{array}{llllll}6+7 & 5+3 & 8+3 & 8+4 & 1+1 & 3+0\end{array}$ $\begin{array}{rrrrrr}5+8 & 6+7 & 7+2 & 5+3 & 3+3 & 0+7\end{array}$ $3+7 \quad 8+4-6+3 \quad 6+7 \quad 4+4 \quad 8+0$ $4+6 \quad 4+5 \quad 5+3 \quad 8+3 \quad 4+4 \quad 6+3$ $\begin{array}{llllll}4+3 & 8+4 & 7+4 & 4+2 & 5+5 & 3+0\end{array}$ $4+7 \quad 3+4 \quad 6+3 \quad 6+1 \quad 6+6 \quad 8+6$

\section{Ex. 2. (1.) \\ (2.) \\ (3.) \\ (4.) \\ (5.) \\ (б.)}

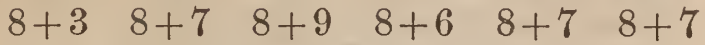

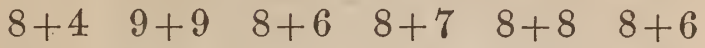

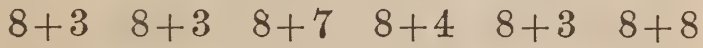

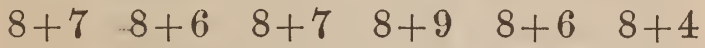

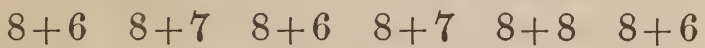
$\begin{array}{lllllll}8+3 & 8+8 & 8+7 & 8+6 & 8+9 & 8+9\end{array}$

\section{Ex. 3. (1.) \\ (2.) \\ (3.) \\ (4.) \\ (5.) \\ (6.)}

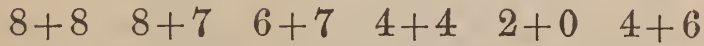
$\begin{array}{llllll}8+3 & 6+7 & 9+3 & 3+3 & 7+0 & 6+0\end{array}$ $\begin{array}{lllllll}3+7 & 6+9 & 8+7 & 2+2 & 8+9 & 0+8\end{array}$ $\begin{array}{lllllll}3+4 & 3+8 & 8+9 & 8+8 & 0+8 & 7+9\end{array}$

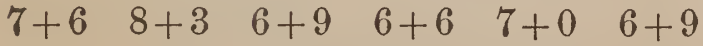
$\begin{array}{llllll}8+9 & 7+4 & 7+8 & 3+3 & 6+8 & 3+8\end{array}$ 


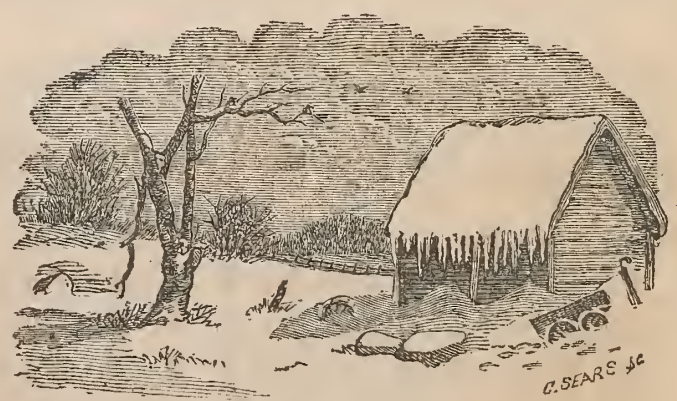

\section{LESSON XXIT.}

One rock and 9 rocks are 10 rocks.

2 trees and 9 trees are 11 trees.

3 wheels and 9 wheels are 12 wheels.

4 carts and 9 carts are 13 carts.

5 limbs and 9 limbs are 14 limbs.

6 sheds and 9 sheds are 15 sheds.

7 icicles and 9 icicles are 16 icicles.

8 posts and 9 posts are 17 posts.

9.crows and 9 crows are 18 crows.

Problems. - If there are 14 icicles on the shed, and there are 7 on one side of the post, how many are on the other? There were 3 crows sitting on the tree, two have flown away; how many remain? Four crows and 5 crows are how many? Six carts and 3 carts are how many? Nine sheds and 4 sheds are how many? Four wheels and 8 wheels are how many? Eight posts and 7 posts are how many? Five trees and 7 trees are how many? Nine rocks and 5 rocks are how many? Four branches and 8 branches are how many? Nine snow-drifts and 3 snow-drifts are how many? Three fences and 5 fences are how many? 
ADDITION.

\section{LESSON XXIII.}

(See Manual, Sec. I., Exercise II.)

Copy, complete, and read the following tables:

Ex. 1.

(2.)

(3.)

(4.)

(5.)

$4+3 \quad 4+3 \quad 4+6 \quad 6+7 \quad 4+6 \quad 4+3$ $\begin{array}{llllll}7+3 & 7+2 & 7+1 & 6+3 & 7+4 & 3+8\end{array}$ $\begin{array}{llllll}6+1 & 6+7 & 6+6 & 5+3 & 3+4 & 8+3\end{array}$ $\begin{array}{llllll}4+2 & 5+7 & 5+6 & 8+6 & 4+3 & 9+3\end{array}$ $\begin{array}{llllll}6+8 & 3+2 & 8+3 & 9+7 & 6+7 & 4+8\end{array}$ $\begin{array}{llllll}3+2 & 6+6 & 4+7 & 7+7 & 6+4 & 7+1\end{array}$

Ex. 2.
(1.)
(2.)
(3.)
(4.)

(5.)

(6.)

$9+4 \quad 4+6 \quad 4+6 \quad 8+6 \quad 9+6 \quad 4+3$ $\begin{array}{llllll}9+3 & 7+3 & 7+8 & 7+9 & 9+8 & 9+9\end{array}$ $\begin{array}{llllll}6+9 & 3+7 & 8+9 & 9+6 & 3+2 & 6+9\end{array}$ $\begin{array}{llllll}3+2 & 8+6 & 6+4 & 9+4 & 9+8 & 3+4\end{array}$ $\begin{array}{llllll}4+6 & 6+4 & 3+4 & 9+8 & 6+4 & 9+2\end{array}$ $\begin{array}{llllll}5+3 & 3+7 & 7+2 & 3+7 & 3+8 & 4+3\end{array}$

Ex. 3.

(2.)

(3.)

(4.)

(5.)

$\begin{array}{llllll}2+2 & 4+2 & 5+6 & 9+9 & 8+8 & 4+6\end{array}$

$\begin{array}{llllll}1+1 & 4+6 & 6+7 & 7+7 & 3+9 & 7+3\end{array}$

$\begin{array}{llllll}6+6 & 6+2 & 8+8 & 6+6 & 7+9 & 3+8\end{array}$

$\begin{array}{llllll}8+9 & 0+0 & 9+9 & 4+4 & 6+8 & 3+8\end{array}$

$\begin{array}{llllll}9+9 & 8+0 & 5+5 & 2+2 & 3+8 & 6+7\end{array}$

$7+7 \quad 0+9 \quad 3+3 \quad 1+1 \quad 2+4 \quad 6+7$ 


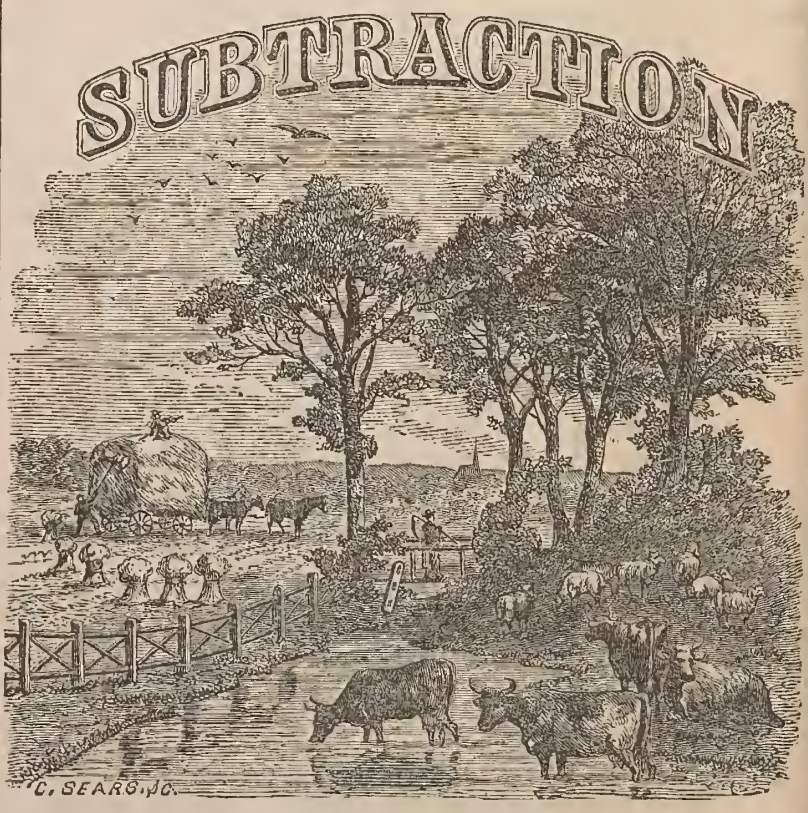

\section{LESSON XXIV.}

One load from 1 load leaves how many?

1 fork from 2 forks leaves how many?

1 horse from 3 horses leaves how many?

1 cow from 4 cows leaves how many?

1 tree from 5 trees leaves how many?

1 sheep from 6 sheep leaves how many?

1 sheaf fiom 7 sheaves leaves how many?

1 post from 8 posts leaves how many?

1 bird from 9 birds leaves how many? 


\section{Sub trace trou}

SUBTRACTION.

\section{LESSON XXV.*}

(See Manual, Sec. I., Exercise III.)

Copy; complete, and read the following tables: Ex. 1. Model.
(1.)
(2)

(3.)

(4.)

$6-1=5 \quad 8-1 \quad 9-1 \quad 8-1 \quad 3-1$

$7-1=6 \quad 6-1 \quad 7-1 \quad 6-1 \quad 7-1$

$9-1=8 \quad 3-1 \quad 8-1 \quad 8-1 \quad 6-1$

$7-1=6 \quad 4-1 \quad 6-1 \quad 2-1 \quad 8-1$

$5-1=4 \quad 9-1 \quad 1-1 \quad 2-1 \quad 3-1$

$3-1=2 \quad 6-1 \quad 4-1 \quad 4-1 \quad 4-1$

Ex. 2. Model.

$3-1=2 \quad 4-1 \quad 5-1 \quad 4-1 \quad 5-1$

$5+1=6 \quad 3-1 \quad 6+1 \quad 3+1 \quad 6+1$

$6+1=-7 \quad 7-1 \quad 7+1 \quad 6-1 \quad 7-1$

$3-1=2 \quad 6-1 \quad 3-1 \quad 7-1 \quad 8+1$

$3+1=4 \quad 3-1 \quad 6+1 \quad 4-1 \quad 0+1$

$5-1=4 \quad 4-1 \quad 5+1 \quad 4+1 \quad 4-1$

Ex. 3 .

(1.)

(2.)

(3.)

(4.)

(5)

(6.)

$4+1 \quad 4-1 \quad 9-1 \quad 1-1 \quad 4-1 \quad 1+9$ $3+1 \quad 6+1 \quad 6-1 \quad 1+1 \quad 3+1 \quad 9-1$ $\begin{array}{llllll}4-1 & 3+1 & 8-1 & 1+0 & 4+0 & 8+7\end{array}$ $\begin{array}{llllll}3-1 & 4-1 & 7+1 & 1+8 & 5-0 & 1+8\end{array}$ $6+1 \quad 7-1 \quad 9-1 \quad 8-1 \quad 6+1 \quad 4-1$ $2+1 \quad 9-1 \quad 3-1 \quad 3-1 \quad 9-1 \quad 9-1$

* Note for the Teacher.-Illustrate the meaning and the use of the sign of subtraction (-) in the following Exercises. 


\section{Thin'u-end}

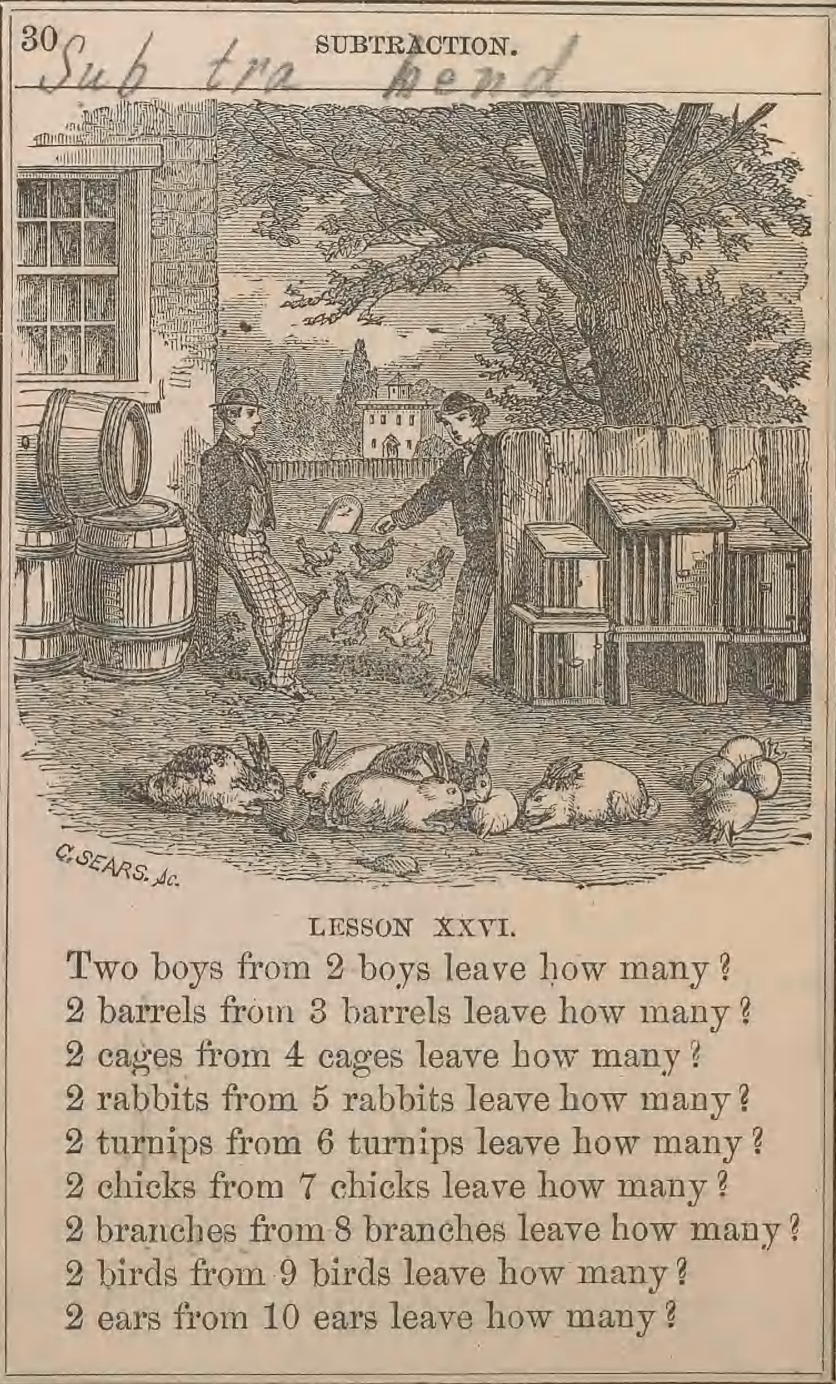




\section{SUBTRACTION.}

\section{IESSON XXVII.}

(See Manual, Sec. I., Exercise III.)

Copy, complete, and read the following tables:

Ex. 1.

(2.)

(3.)

(4.)

(5.)

(6.)

(3) $3-2 \quad 4-2 \quad 4-2 \quad 4-2 \quad 4-2 \quad 4-2$

$6-2 \quad 6-2 \quad 6-2 \quad 6-2 \quad 6-2 \quad 8-2$

4-2 $7-2 \quad 3-2 \quad 8-2 \quad 4-2 \quad 7-2$

$3-2 \quad 3-2 \quad 4-2 \quad 4-2 \quad 3-2 \quad 3-2$

$\begin{array}{llllll}5-2 & 4-2 & 6-2 & 3-2 & 4-2 & 4-2\end{array}$

$6-2 \quad 6-2 \quad 4-2 \quad 6-2 \quad 6-2 \quad 5-2$

Ex, 2. (1.) (2.) (3.) (4.) (5.) (6.)

$2-2 \quad 4-2 \quad 4-2 \quad 4-2 \quad 5-2 \quad 4-2$

$4-2 \cdot 6-2 \quad 7-2 \quad 6-2 \quad 7-2 \quad 6-2$

$6-2 \quad 4-2 \quad 4-2 \quad 3-2 \quad 3-2 \quad 3-2$

$\begin{array}{llllll}3-2 & 3-2 & 6-2 & 4-2 & 6-2 & 2-2\end{array}$

$6-2 \quad 8-2 \quad 8-2 \quad 6-2 \quad 4-2 \quad 3-2$

$3-2 \quad 6-2 \quad 3-2 \quad 4-2 \quad 3-2 \quad 6-2$

Ex. 3. (1.)

(2.)

(3.)

(4.)

(5.)

(6.)

$2-2 \quad 6-2 \quad 4-2 \quad 9-2 \quad 6-2 \quad 9+2$

$\begin{array}{llllll}8+2 & 8-2 & 7+2 & 7+2 & 8+2 & 7+2\end{array}$

$7+2 \quad 4+2 \quad 4-2 \quad 8-2 \quad 2-2 \quad 10+2$

$\begin{array}{llllll}4-2 & 7+2 & 8+2 & 6+2 & 7+2 & 11-2\end{array}$

$\begin{array}{llllll}6+2 & 3-2 & 7-2 & 8+2 & 11+2 & 12+2\end{array}$

$\begin{array}{llllll}4-2 & 8+2 & 6+2 & 4-2 & 11+2 & 7+2\end{array}$ 


$$
32 \text { SUBTRACTION. }
$$

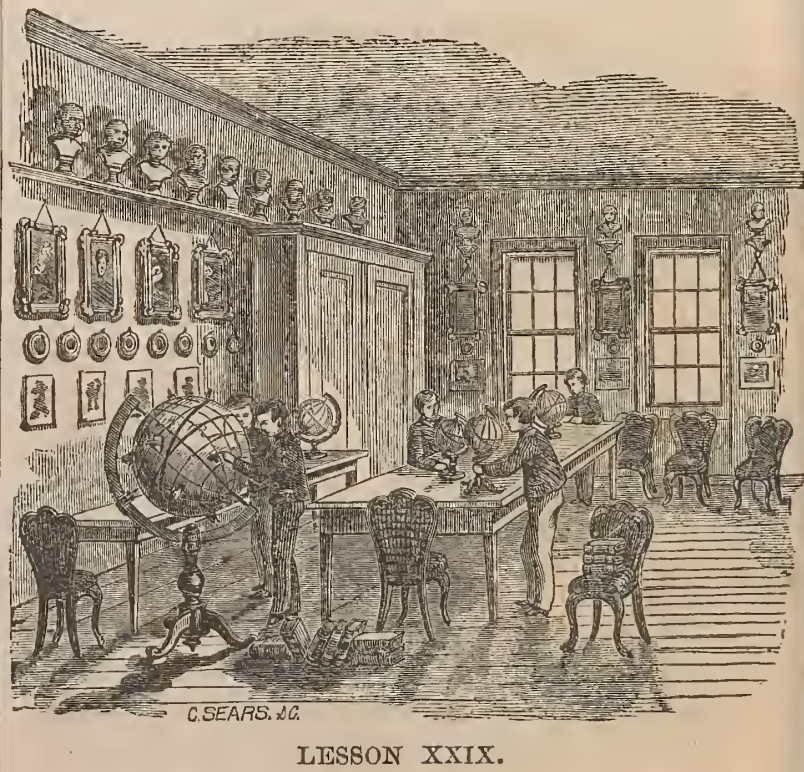

3 books from 3 books leave how many?

3 pictures from 4 pictures leave liow many?

3 globes from 5 globes leave how many?

3 chairs from 6 chairs leave how many?

3 books from 7 books leave how many?

3 pictures from 8 pictures leave how many?

3 busts from 9 busts leave how many?

3 books from 10 books leave how many?

3 pictures from 11 pictures leave how many?

3 busts from 12 busts leave how many? 


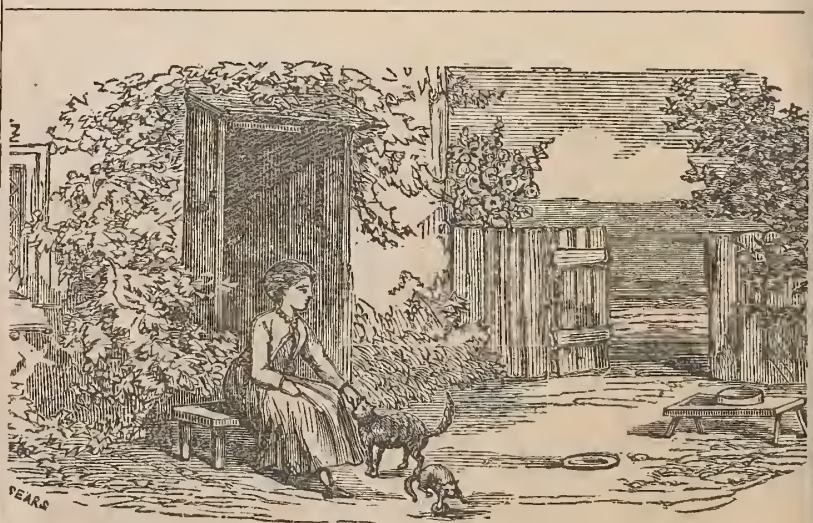

\section{LESSON XXX.}

4 houses from 4 houses leave no houses.

4 roses from 5 roses leave 1 rose.

4 gates from 6 gates leave 2 gates.

4 girls from 7 girls leave 3 girls.

4 kittens from 8 kittens leave 4 kittens.

4 dishes from 9 dishes leave 5 dishes.

4 stools from 10 stools leave 6 stools.

4 cats from 11 cats leave 7 cats.

4 bushes from 12 bushes leave 8 bushes.

4 windows from 13 windows leave 9 windows.

* Problems.-There are two stools, each has 4 legs; how many legs have both? There are 7 little girls at a picnic, 4 of them return; how many remain? There are 8 roses on the rose-bush; Henry picked 4 of them; how many remain? etc.

* These and the following problems are not for the pupils to sludy, but for the teacher to dictate to the class. 


\section{LESSON $\times X \times I$.}

(See Manual, Sec. I., Exercise III.)

Copy, complete, and read the following tables:

Ex. 1.

(2.)

(3.)

(4.)

(5.)

(6.)

$6-4 \quad 5-4 \quad 8-4 \quad 7-4 \quad 9-4 \quad 8-4$ $\begin{array}{llllll}7-4 & 6-4 & 6-4 & 8-4 & 8-4 & 7-4\end{array}$ $8-4 \quad 9-4 \quad 8-4 \quad 6-4 \quad 6-4 \quad 6-4$ $9-4 \quad 4-4 \quad 7-4 \quad 13-4 \quad 8-4 \quad 8-4$ $8-4 \quad 6-4 \quad 6-4 \quad 4-4 \quad 9-4 \quad 8-4$ $\begin{array}{llllll}6-4 & 7-4 & 9-4 & 7-4 & 11-4 & 7-4\end{array}$

Ex. 2 . (1.)

(2.)

(3.)

(4.)

(5.)

(6.)

$4-3 \quad 8-4 \quad 8-4 \quad 7-4 \quad 13-411-4$ $8-4 \quad 7-4 \quad 11-4 \quad 6-4 \quad 10-4 \quad 13-4$ $7-4 \quad 11-4 \quad 12-4 \quad 8-4 \quad 8-4 \quad 10-4$ $9-4 \quad 9-4 \quad 13-4 \quad 4-4 \quad 11-4 \quad 9-4$ $\begin{array}{llllll}6-4 & 8-4 & 11-4 & 8-4 & 7-4 & 12-4\end{array}$ $\begin{array}{llllll}12-4 & 10-4 & 8-4 & 7-4 & 6-4 & 4-4\end{array}$

Ex. 3.
(1.)
(2.)

(3.)

(4.)

(5.) (6.)

$8+4 \quad 3+4 \quad 8+4 \quad 8-4 \quad 9+4 \quad 4-4$ $\begin{array}{llllll}4-4 & 7+4 & 4-4 & 8+4 & 7+4 & 8+6\end{array}$ $\begin{array}{llllll}6-4 & 6-4 & 8-4 & 7-4 & 13-4 & 4-4\end{array}$ $7+4 \quad 8-4 \quad 8+4 \quad 3+4 \quad 10-4 \quad 8+4$ $\begin{array}{lllllll}3+4 & 9+4 & 5+4 & 4+6 & 11-4 & 7+4\end{array}$ $6+7 \quad 6+4 \quad 7-4 \quad 13-4 \quad 6+4 \quad 13-4$ 


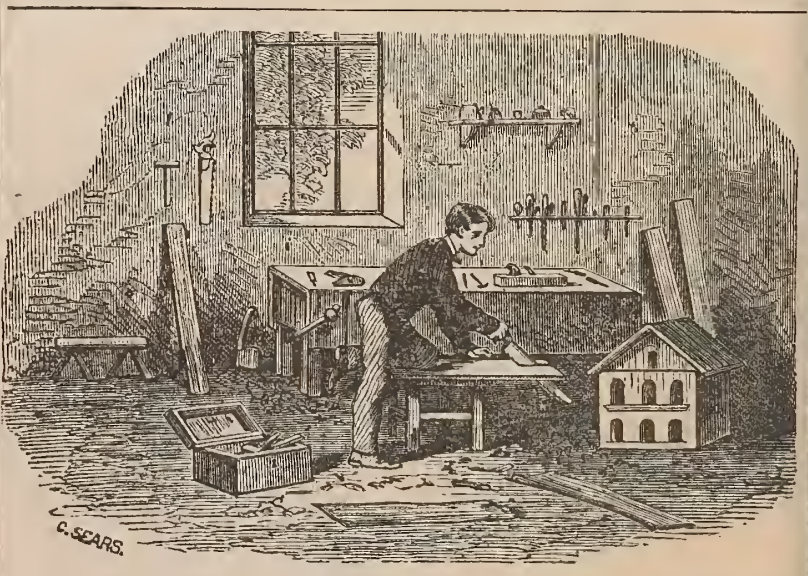

\section{LESSON X்XXII.}

5 saws from 5 saws leave no saws.

5 boxes from 6 boxes leave 1 box.

5 planes from 7 planes leave 2 planes.

5 hammers from 8 hammers leave 3 hammers.

5 chisels from 9 chisels leave 4 chisels.

5 boards from 10 boards leave 5 boards.

5 benches from 11 benches leave 6 benches.

5 axes from 12 axes leave 7 axes.

5 rules from 13 rules leave 8 rules.

5 cages from 14 cages leave 9 cages.

Problems. $-\Lambda$ little boy agreed to make 8 bird-cages; he has 4 of them done; how many has he jet to make? He had 11 chisels, but broke 3 of them; how many had he left? He used 5 pieces of board, and has 4 left; how many had he at first? He had 8 augers; he loaned three of them; how many had he left? etc. 


\section{IESSON XXXIII.}

(See Manual, Sec. I., Exercise III.)

Copy, complete, and read the following tables:

Ex. 1.

(2.)

(3.)

(4.)

(5.)

(6.)

r. $6-5 \quad 4-5 \quad 10-5 \quad 8-5 \quad 14-5 \quad 14-5$ $\begin{array}{llllll}7-5 & 6-5 & 8-5 & 7-5 & 9-5 & 6-5\end{array}$

$9-5 \quad 7-5 \quad 7-5 \quad 13-5 \quad 7-5 \quad 8-5$ $\begin{array}{llllll}10-5 & 8-5 & 6-5 & 14-5 & 6-5 & 9-5\end{array}$

$8-5 \quad 6-5 \quad 8-5 \quad 6-5 \quad 8-5 \quad 11-5$ $7-5 \quad 5-5 \quad 9-5 \quad 5-5 \quad 13-5 \quad 6-5$

Ex. 2 .
(1.)

(2.)

(3.)

(4.)

(5.)

(6.)

$8-511-5 \quad 13-5 \quad 14-5 \quad 14-5 \quad 7-5$ $\begin{array}{llllll}7-5 & 7-5 & 6-5 & 7-5 & 8-5 & 6-5\end{array}$ $6-5 \quad 11-5 \quad 8-5 \quad 8-5 \quad 6-5 \quad 7-5$ $\begin{array}{llllll}9-5 & 12-5 & 8-5 & 8-5 & 8-5 & 9-5\end{array}$ $8-5 \quad 13-5 \quad 7-5 \quad 13-5 \quad 7-5 \quad 9-5$ $\begin{array}{llllll}13-5 & 14-5 & 6-5 & 6-5 & 8-5 & 7-5\end{array}$

Ex. 3.

(1.)

(2.)

(3.)

(4.)

(5.)

(6.)

$3+5 \quad 4+5 \quad 8+1 \quad 8-2 \quad 5+6 \quad 5-5$ $\begin{array}{llllll}7+5 & 7-5 & 9+2 & 3+8 & 7+4 & 7-4\end{array}$ $\begin{array}{llllll}9-5 & 3+5 & 7-2 & 7-5 & 7-2 & 4-4\end{array}$ $\begin{array}{llllll}13-5 & 2+5 & 7+2 & 2+5 & 7+2 & 0-0\end{array}$

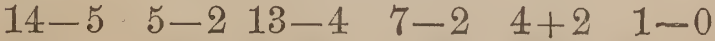
$\begin{array}{llllll}5+3 & 7-5 & 7-4 & 3+4 & 2+3 & 1-1\end{array}$ 


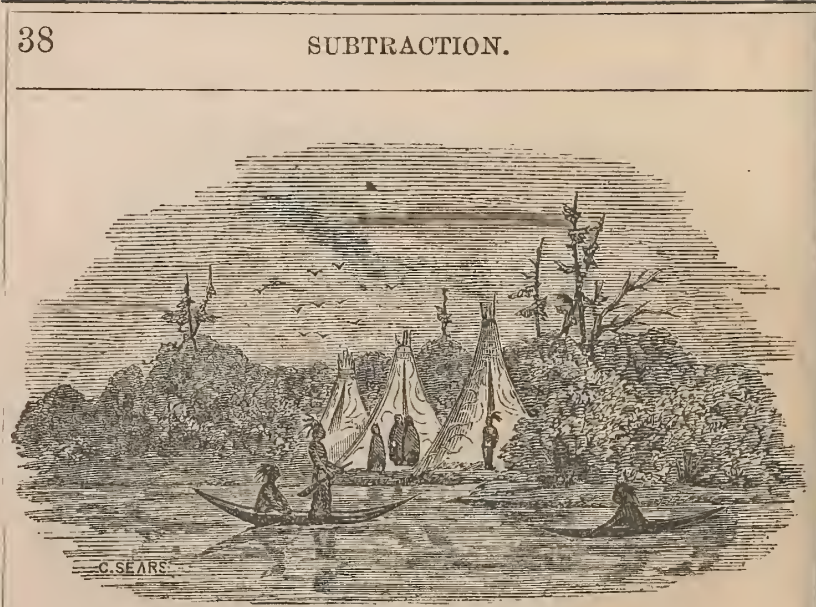

LIESSON XXXIV.

6 tents from 6 tents leave no tents. .

6 Indians from 7 In $r^{r}$ ians leave 1 Indian.

6 canoes from 8 canoes leave 2 canoes.

6 paddles from 9 paddles leave 3 paddles.

6 trees from 10 trees leave 4 trees.

6 buishels from 11 bushels leave 5 bushels.

6 hirds from 12 birds leave 6 birds.

6 bows from 13 bows leave 7 bows.

6 arrows from 14 arrows leave 8 arrows.

6 spears from 15 spears leave 9 spears.

Problems.-A party of 12 Indians went to hunt; 6 of them returned; how many were still away'? In a village there were 11 wigwams, all but 3 were blown down; how many were blown down? There are 3 Indians in the boats; and 1 is standing on the shore; how many are there in all? etc. 


\section{LESSON XXXV.}

(See Manual, Sec. I., Exercise III.)

Copy, complete, and read the following tables:

Ex. 1 .

(1.)

(2.)

(3.)

(4.)

(5.)

(6.)

$14-69-615-6 \quad 14-6 \quad 9-614-6$

$14-6 \quad 7-6 \quad 11-6 \quad 7-6 \quad 8-6 \quad 13-6$

$15-6 \quad 9-6 \quad 8-6 \quad 8-6 \quad 7-6 \quad 7-6$

$11-612-615-614-610-6 \quad 8-6$

$14-613-6 \quad 13-6 \quad 15-6 \quad 13-6 \quad 14-6$

$13-613-611-612-615-69-6$

Ex. 2.

(2.) (3.)

(4.)

(5.).

(6.)

$5+6 \quad 7-6 \quad 5-3 \quad 5+7 \quad 15-6 \quad s+6$ $7-6 \quad 8-6 \quad 5-3 \quad 3-3 \quad 13-6 \quad 7+6$ $\begin{array}{llllllll}4+2 & 8+2 & 8+3 & 13 & -5 & 14-6 & 7-6\end{array}$ $8+27+6 \quad 6+315-5 \quad 8-0 \quad 5-2$ $3+1 \quad 3-2 \quad 7-2 \quad 0-0 \quad 7+3 \quad 3-1$ $6+1 \quad 3+6 \quad 6-4 \quad 7-3 \quad 5+3 \quad 6-6$

Ex. 3. (1.)

(2.)

(3.)

(4.)

(5.)

(6.)

$7-6 \quad 8+69+6 \quad 15-6 \quad 13-6 \quad 11-6$

$9+6113-611-6 \quad 9-5 \quad 7+512-6$

$8+611-613-611-6 \quad 6+613-6$

$15-615-614-610-614-69+6$

$13-6 \quad 12-613-6 \quad 13-6 \quad 13-6 \quad 15-6$

$11-615-610-614-6 \quad 9+6 \quad 6+6$ 

40
SUBTRACTION.

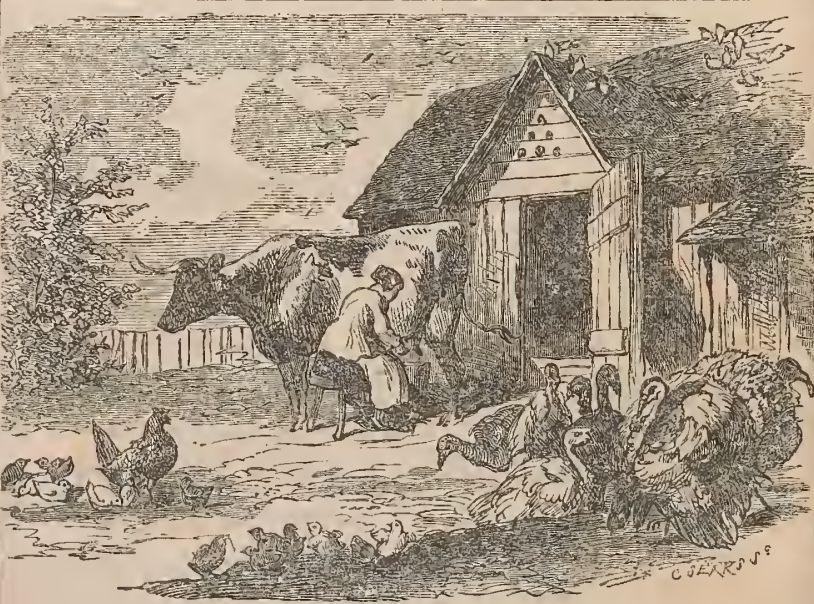

LESSON XXXVI.

7 turkeys from 7 turkeys leave how many?

7 turkeys from 8 turkeys leave how many?

7 chicks from 9 chicks leave 2 chicks.

7 doves from 10 doves leave 3 doves.

7 cows from 11 cows leave 4 cows.

5 hens from 12 hens leave 5 hens.

7 swallows from 13 swallows leave 6 swallows.

7 pails from 14 pails leave 7 pails.

7 shieds from 15 sheds leave 8 sheds.

7 barns from 16 barns leave 9 barns.

Pkoblems. - There are 6 chicks and 1 chick in a brood; bow many chicks are there? There are 13 swallows in a flock, 7 of them have flown away; how many remain? etc. 


\section{ESSON XXXVII.}

(See Manual, Sec. I., Exercise III.)

Copy, complete, and read the following tables:

cx. 1.

(1.)

(2.)

(3.)

(4.)

(5)

(6.)

$\begin{array}{llllll}10-7 & 11-7 & 14-7 & 14-7 & 7-7 & 8-7\end{array}$

$\begin{array}{lllllll}16-7 & 8-7 & 16-7 & 13-7 & 16-7 & 9-7\end{array}$

$\begin{array}{llllll}13-7 & 9-7 & 15-7 & 16-7 & 8-7 & 13-7\end{array}$

$\begin{array}{llllll}11-7 & 11-7 & 8-7 & 15-7 & 9-7 & 16-7\end{array}$

$\begin{array}{llllll}16-7 & 14-7 & 16-7 & 8-7 & 16-7 & 15-7\end{array}$

$\begin{array}{llllll}14-7 & 16-7 & 13-7 & 9-7 & 15-7 & 8-7\end{array}$

Ex. 2.

(2.)

(3.)

(4.)

(5.)

(6.)

$7-1 \quad 7+7 \quad 13-7 \quad 15-7 \quad 10-7 \quad 15-7$

$7+7 \quad 9+7 \quad 14-7 \quad 16-7 \quad 16-7 \quad 16-7$

$\begin{array}{llllllll}7-3 & 9-7 & 15-7 & 13-7 & 15-7 & 11-7\end{array}$

$7+410+716-711-713-612-7$

$7+5 \quad 11-7 \quad 15-7 \quad 12-7 \quad 11-7 \quad 9-7$

$7-612-7 \quad 7+0 \quad 11-7 \quad 14-7 \quad 7+7$

Ex. 3.

(1.)

(2.)

(3.)

(4.)

(5.)

(6.)

$7+213-6 \quad 8+3 \quad 8+3 \quad 8+7 \quad 4+5$

$\begin{array}{lllll}7-1 & 3+6 & 7-2 & 7+1 & 5+9\end{array}$

$8-1 \quad 4+6 \quad 8-3 \quad 5-2 \quad 6-3 \quad 3+8$

$\begin{array}{llllll}4-2 & 7+5 & 7-4 & 5+2 & 9-7 & 9-7\end{array}$

$2+2 \quad 5-2 \quad 6+2 \cdot 9-7 \quad 13-7 \quad 3-1$

$5+2 \quad 5+2 \quad 4+3 \quad 6+6 \quad 16-7 \quad 8-2$ 


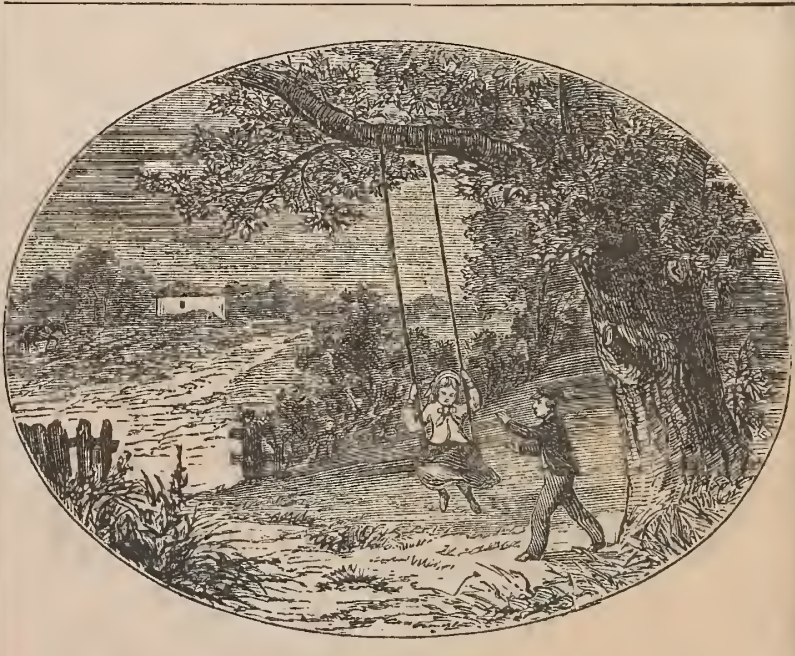

\section{LESSON XXXVII.}

Eight trees from 8 trees leave no trees. 8 ropes from 9 ropes leave 1 rope. 8 boys from 10 boys leave 2 boys. 8 girls from 11 girls leave 3 girls. 8 houses from 12 houses leave 4 houses. 8 barns from 13 barns leave 5 barns. 8 caps from 14 caps leave 6 caps. 8 flowers from 15 flowers leave 7 flowers.

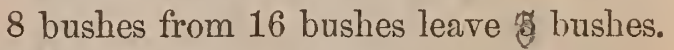
8 horses from 17 horses leave 9 horses.

Problems.-John swung his little sister 5 minutes and his little brother 7 minutes; how many minutes did he swing both? 


\section{LESSON XXXIX.}

\section{(See Manual, Sec. I., Exercise III.)}

Copy, complete, and read the following tables:

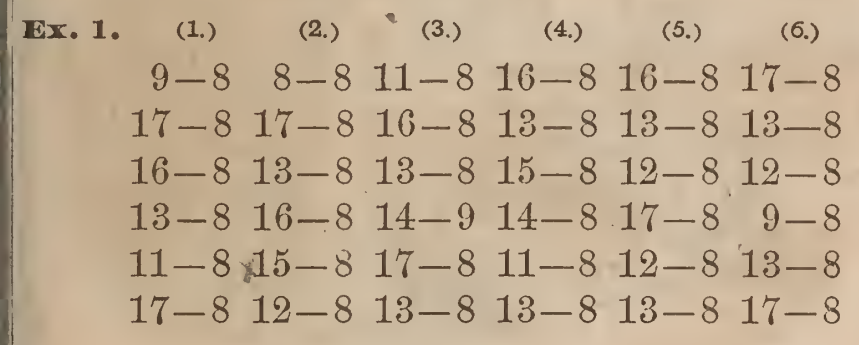

Ex. 2.

(1.)

(2.)

(3.)

(4.)

(5.)

(6.)

$\begin{array}{llllll}17-8 & 14-8 & 9-8 & 8-2 & 13-8 & 14-8\end{array}$

$\begin{array}{lllllll}11-8 & 13-8 & 8+7 & 8+1 & 15-8 & 17-8\end{array}$

$\begin{array}{lllllll}16-8 & 12-8 & 8+6 & 8-0 & 16-8 & 16-8\end{array}$

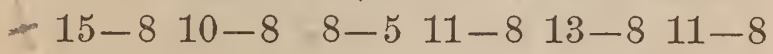

$\begin{array}{llllll}14-8 & 11-8 & 8+4 & 13-8 & 11-8 & 12-8\end{array}$

$13-8 \quad 8+8 \quad 8+3 \quad 15-8 \quad 13-8 \quad 13-8$

Ex. 3. (1.) (2.) (3.) $\quad$ (4.) $\quad$ (5.)

$\begin{array}{llllll}17-8 & 6+9 & 5+8 & 8-8 & 5+8 & 8+0\end{array}$ $\begin{array}{llllll}16-8 & 11-8 & 13-8 & 8+8 & 9-8 & 0+8\end{array}$ $13-8 \quad 8-8 \quad 6+8 \quad 3+8 \quad 13-8 \quad 8-8$ $14-8 \quad 8+8 \quad 14-8 \quad 13-8 \quad 17-8 \quad 8-0$

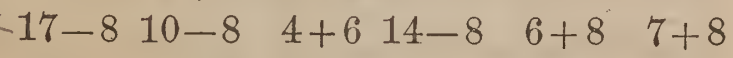
$\begin{array}{llllll}13-8 & 9+8 & 5+7 & 4+7 & 5+8 & 8+8\end{array}$ 


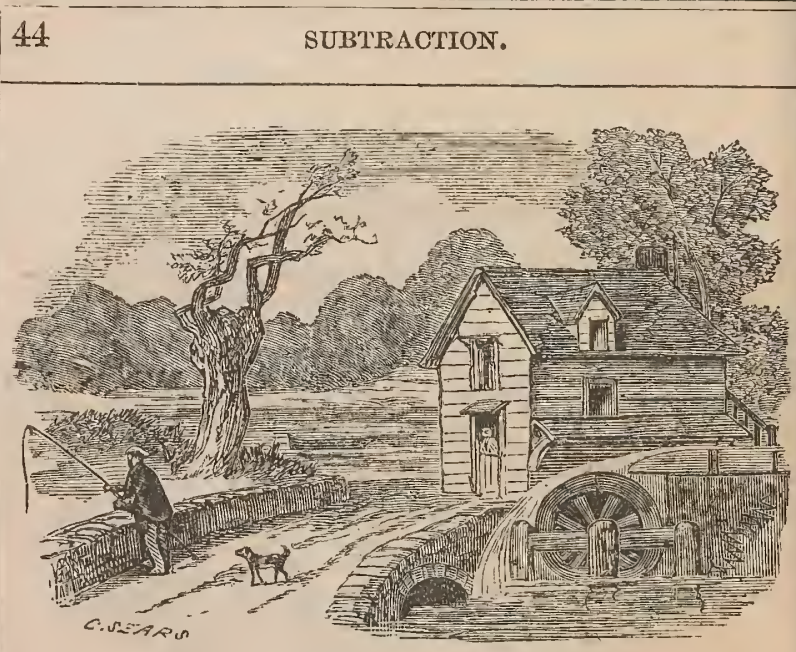

\section{LESSON XL。}

9 mills froin 9 mills leave no mills.

9 trees from 10 trees leave 1 tree.

9 men from 11 men leave 2 men.

9 boats from 12 boats leave 3 boats.

9 dogs from 13 dogs leave 4 dogs.

9 wheels from 14 wheels leave 5 wheels.

9 doors from 15 doors leave 6 doors.

9 bridǵes from 16 bridges leave 7 bridges.

9 fish-poles from 17 tish-poles leave 8 fish-poles. 9 posts from 18 posts leave 9 posts.

Problenr.-A man caught 8 fishes at one time and 6 at another; how many did he catch in all? If a mill grinds 8 bushels of corn and 12 bushels of wheat; how much more wheat does it grind than corn? 


\section{LESSON XLI.}

\section{'(See Manual, Sec. I., Exercise III.)}

Copy, read, and complete the following tables:

$$
\text { Ex. 1. } \begin{array}{llll|cc}
\text { (1.) } & \text { (2.) } & \text { (3.) } & \text { (4.) } & \text { (5.) } & \text { (6.) } \\
9-9 & 9-3 & 15-9 & 13-9 & 14-9 & 15-9 \\
9-8 & 9-2 & 18-9 & 14-9 & 13-9 & 16-9 \\
9-7 & 9-1 & 17-9 & 16-9 & 18-9 & 18-9 \\
9-6 & 9-0 & 16-9 & 18-9 & 16-9 & 13-9 \\
9-5 & 10-9 & 18-9 & 13-9 & 17-9 & 15-9 \\
9-4 & 11-9 & 17-9 & 10-9 & 9-9 & 18-9
\end{array}
$$
Ex.2.
(2.)
(3.)
(4.)
(5.)
(6.)

$8+7 \quad 4-3 \quad 4+6 \quad 3+7 \quad 4+6 \quad 4+6$ $5+6 \quad 9-4 \quad 3+7 \quad 8+9 \quad 13-9 \quad 8+6$ $\begin{array}{lllllll}3-2 & 9+3 & 18-9 & 3-1 & 14-8 & 9+1\end{array}$ $\begin{array}{llllll}8-4 & 8+7 & 17-8 & 0+1 & 7+8 & 3+1\end{array}$ $\begin{array}{lllllll}9-3 & 4+3 & 4+7 & 8+0 & 9+5 & 5-2\end{array}$ $\begin{array}{llllll}7+6 & 8+7 & 6+9 & 9-3 & 11-9 & 7-2\end{array}$
Ex. 3.
(1.)
(2.)
(3.)
(4.)
(5.)
(6.)

$9+9 \quad 9+1 \quad 8+8 \quad 4+9 \quad 15-9 \quad 4+8$ $\begin{array}{llllll}13-9 & 18-9 & 7+9 & 7+8 & 7+9 & 7+9\end{array}$ $\begin{array}{llllll}18-9 & 17-9 & 11-9 & 9-8 & 8+9 & 6+9\end{array}$

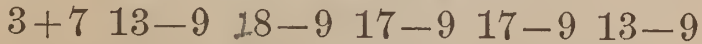
$7+9 \quad 6+9 \quad 17-9 \quad 11-9 \quad 16-9 \quad 15-9$ $18-9 \quad 3+9 \quad 13-9 \quad 13-9 \quad 13-9 \quad 17-9$ 


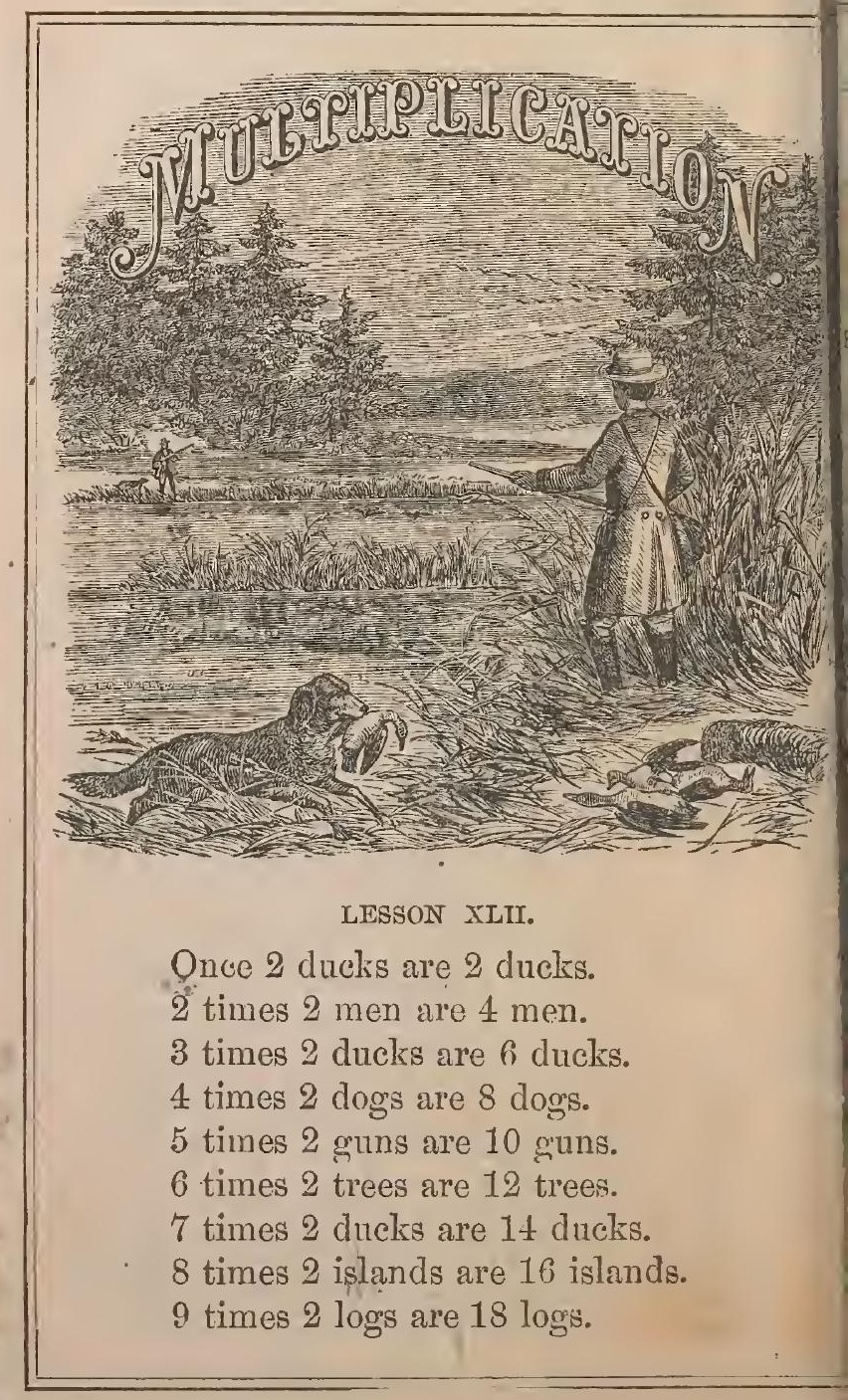


MULTIPLICATION.

\section{ESSON XIIII.*}

(See Manual, Sec. I., Exercise IV.)

Copy, complete, and read the following tables:

Ex. 1. ' Model.

(1.)

(2.)

(3.)

(4.)

$4 \times 2=8 \quad 2 \times 2 \quad 2 \times 2 \quad 5 \times 2 \quad 4 \times 2$

$3 \times 2=6 \quad 0 \times 2 \quad 3 \times 2 \quad 4 \times 2 \quad 5 \times 2$

$2 \times 2=4 \quad 3 \times 2: 4 \times 2 \quad 1 \times 2 \quad 3 \times 2$

$\begin{array}{lllll}1 \times 2=2 & 2 \times 3 & 1 \times 2 & 1 \times 1 & 2 \times 2\end{array}$

$\begin{array}{lllll}5 \times 2=10 & 3 \times 2 & 3 \times 2 & 2 \times 2 & 4 \times 2\end{array}$

$4 \times 2=8 \quad 4 \times 2 \quad 4 \times 2 \quad 3 \times 2 \quad 1 \times 2$

Ex. 2. Model.

(1.)

(2.)

(3.)

(4.)

$3 \times 2=6 \quad 6 \times 2 \quad 1 \times 2 \quad 6 \times 2 \quad 4 \times 2$

$4 \times 2=8 \quad 7 \times 2 \quad 7 \times 2 \quad 7 \times 2 \quad 3 \times 2$

$5 \times 2=10 \quad 6 \times 2 \quad 3 \times 2 \quad 6 \times 2 \quad 9 \times 2$

$7 \times 2=14 \quad 7 \times 2 \quad 6 \times 2 \quad 3 \times 2 \quad 6 \times 2$

$2 \times 2=4 \quad 4 \times 2 \quad 8 \times 2 \quad 8 \times 2 \quad 9 \times 2$

$0 \times 2=0 \quad 4 \times 0 \quad 3 \times 2 \quad 6 \times 2 \quad 3 \times 2$

ERx. 3.

(1.)

(2.)

(3.)

(4.)

(5.)

(6.)

$\begin{array}{llllll}3 \times 2 & 5+2 & 5 \times 2 & 4+2 & 0 \times 6 & 7+2\end{array}$

$2+3 \quad 7-2 \quad 5-2 \quad 6+2 \quad 6 \times 0 \quad 4-2$

$\begin{array}{llllll}4 \times 2 & 3 \times 2 & 5+2 & 2+6 & 6-0 & 7+2\end{array}$

$\begin{array}{llllll}6-2 & 7 \times 2 & 2+5 & 2 \times 6 & 6+0 & 9-1\end{array}$

$2 \times 6 \quad 6-2 \quad 3+0 \quad 6 \times 2 \quad 0+6 \quad 8-2$

$\begin{array}{llllll}4-2 & 4+2 & 0+3 & 6-0 & 9 \times 2 & 4-2\end{array}$

* Note for the Teacher.-Illustrate the meaning and the use of the sign ff multiplication $(x)$ in the following Exercises. 


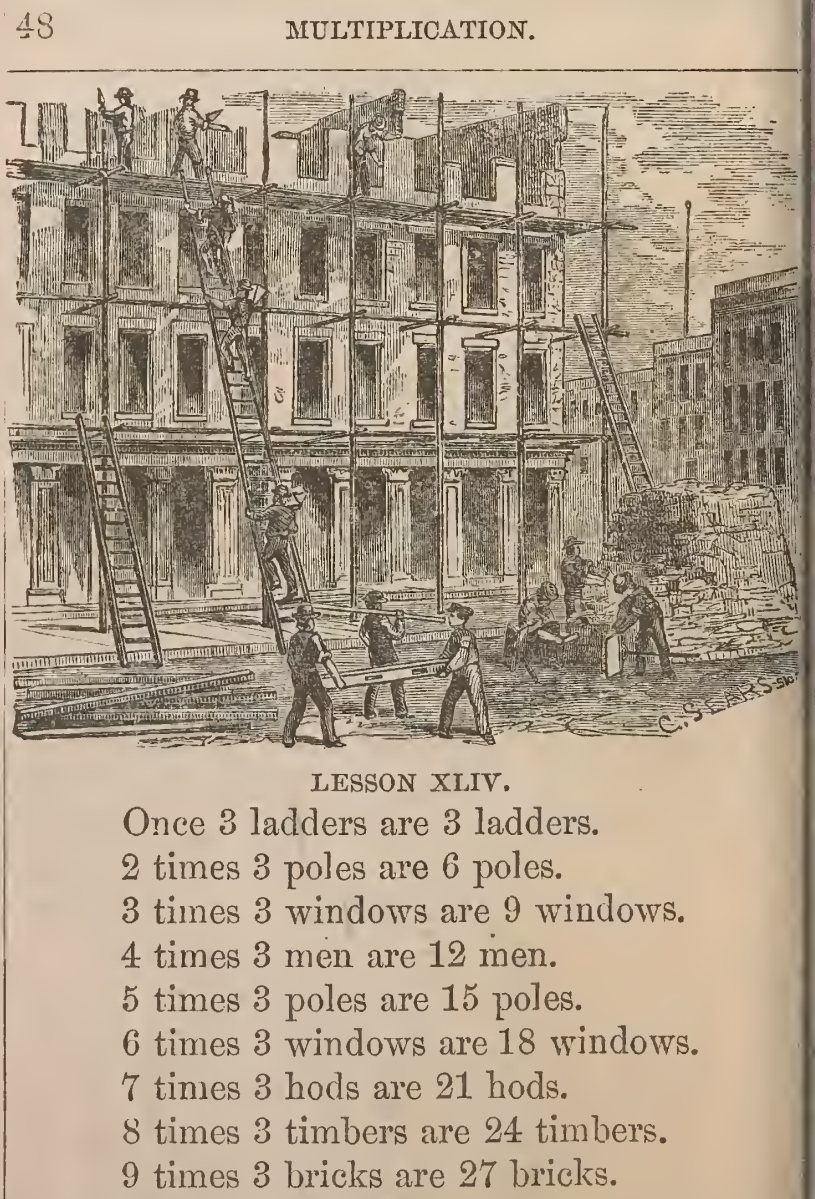

Prorlears.-If a man carry 9 bricks in his hod at one load, ho many bricks can he carry in going 3 times? There are 9 window in one story; how many windows in 3 stories? 


\section{IESSON XIV.}

(See Manual, Sec. I., Exercise IV.)

Copy, complete, and read the following tables:

\begin{tabular}{|c|c|c|c|c|c|c|}
\hline Ex. 1. & (1.) & (2.) & (3.) & (4.) & (5.) & (6.) \\
\hline & $5 \times 3$ & $1 \times 3$ & $2 \times 3$ & $4 \times 3$ & $6 \times 3$ & $3 \times 6$ \\
\hline & $4 \times 3$ & $7 \times 3$ & $3 \times 3$ & $5 \times 3$ & $7 \times 3$ & $3 \times 2$ \\
\hline & $3 \times 3$ & $5 \times 3$ & $0 \times 3$ & $4 \times 3$ & $8 \times 3$ & $3 \times 5$ \\
\hline & $2 \times 3$ & $2 \times 3$ & $5 \times 3$ & $1 \times 3$ & $5 \times 3$ & $5 \times 3$ \\
\hline & $4 \times 3$ & $4 \times 3$ & $8 \times 3$ & $0 \times 3$ & $7 \times 3$ & $4 \times 3$ \\
\hline & $5 \times 3$ & $2 \times 3$ & $6 \times 3$ & $3 \times 0$ & $6 \times 3$ & $2 \times 3$ \\
\hline
\end{tabular}

Ex. 2.

(2.)

(3.)

(4.)

(5.)

(6.)

$\begin{array}{llllll}4 \times 3 & 5 \times 3 \quad 7 \times 3 & 1 \times 3 & 5 \times 3 & 6 \times 3\end{array}$ $5 \times 3 \quad 7 \times 3 \quad 6 \times 3 \quad 9 \times 4] 7 \times 3 \quad 7 \times 3$ $\begin{array}{llllll}7 \times 3 & 6 \times 3 & 4 \times 3 & 6 \times 3 & 6 \times 3 & 6 \times 3\end{array}$ $\begin{array}{llllll}8 \times 3 & 3 \times 3 & 0 \times 3 \quad 7 \times 3 & 8 \times 3 \quad 8 \times 3\end{array}$ $\begin{array}{llllll}4 \times 3 & 7 \times 3 & 6 \times 3 & 5 \times 3 & 7 \times 3 & 6 \times 3\end{array}$ $\begin{array}{llllll}6 \times 3 & 9 \times 3 & 7 \times 3 & 6 \times 3 & 9 \times 3 & 9 \times 3\end{array}$

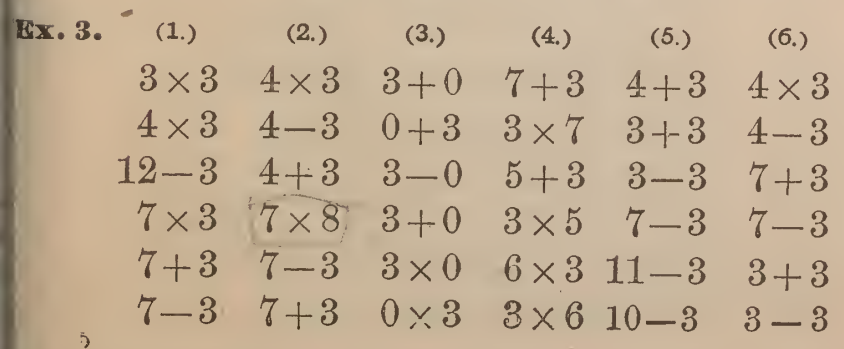




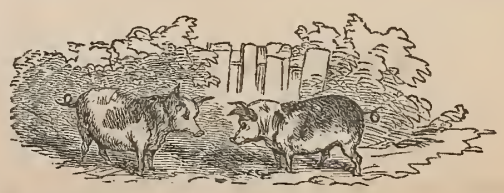

4 times 2 pigs are 8 pigs.

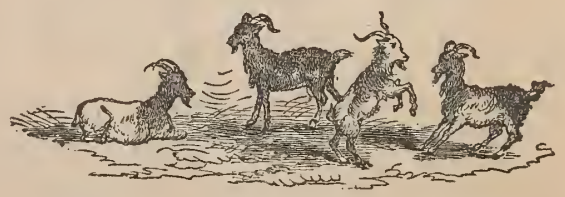

4 times 4 goats are 16 goats.

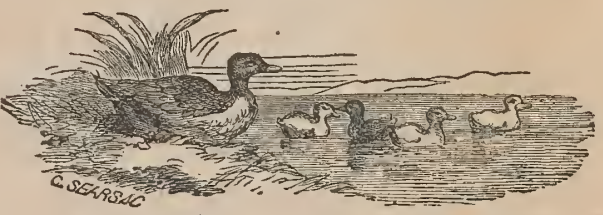

5 times 5 ducks are 25 ducks.

\section{LESSON XLVI.}

Once 4 goats are 4 goats. 2 times 4 feet are 8 feet.

3 times 4 nests are 12 nests. 4 times 4 birds are 16 birds. 5 times 4 pigs are 20 pigs. 6 times 4 kids are 24 kids. 7 times 4 wings are 28 wings. 8 times 4 eggs are 32 eggs. 9 times 4 ears are 36 ears. 


\section{LESSON XLVII.}

(See Manual, Sec. I., Exercise IV.)

Copy, read, and complete the following tables:
Ex. 1.
(2.)
(3.)
(4.)
(5.)
(6.)

$\begin{array}{llllll}3 \times 4 & 6 \times 4 & 6 \times 4 & 3 \times 4 & 6 \times 4 & 4 \times 4\end{array}$ $\begin{array}{llllll}2 \times 4 & 2 \times 4 & 7 \times 4 & 2 \times 4 & 3 \times 4 & 6 \times 4\end{array}$ $\begin{array}{llllll}0 \times 4 & 7 \times 4 & 3 \times 6 & 4 \times 4 & 7 \times 4 & 3 \times 4\end{array}$ $\begin{array}{llllll}1 \times 4 & 9 \times 4 & 8 \times 4 & 0 \times 4 & 3 \times 4 & 7 \times 4\end{array}$ $\begin{array}{llllll}8 \times 4 & 3 \times 4 & 2 \times 4 & 3 \times 4 & 6 \times 4 & 6 \times 4\end{array}$ $\begin{array}{llllll}3 \times 4 & 2 \times 4 & 7 \times 4 & 3 \times 4 & 2 \times 4 & 3 \times 4\end{array}$
Ex. 2. (1.)
(2.)
(3.)
(4.)
(5.)
(6.)

$\begin{array}{llllll}3 \times 4 & 5 \times 4 & 8 \times 4 & 6 \times 4 & 8 \times 4 & 5 \times 4\end{array}$ $\begin{array}{llllll}4 \times 4 & 3 \times 4 & 7 \times 4 & 5 \times 4 & 6 \times 4 & 7 \times 4\end{array}$ $\begin{array}{llllll}7 \times 4 & 7 \times 4 & 8 \times 4 & 7 \times 4 & 8 \times 4 & 8 \times 4\end{array}$ $\begin{array}{llllll}9 \times 4 & 5 \times 4 & 0 \times 4 & 3 \times 4 & 9 \times 4 & 3 \times 4\end{array}$ $\begin{array}{llllll}6 \times 4 & 8 \times 4 & 9 \times 4 & 6 \times 4 & 7 \times 4 & 7 \times 4\end{array}$ $\begin{array}{llllll}4 \times 4 & 7 \times 4 & 7 \times 4 & 7 \times 4 & 6 \times 4 & 6 \times 4\end{array}$
Ex. 3. (1.)
(2.)
(3.)
(4.)
(5.)

(6.)

$\begin{array}{llllll}3+4 & 5 \times 4 & 6+4 & 8-4 & 6 \times 4 & 2+4\end{array}$ $\begin{array}{llllll}3 \times 4 & 5-4 & 6-4 & 8-0 & 6+4 & 2-1\end{array}$ $\begin{array}{llllll}3-0 & 5+4 & 6 \times 4 & 0 \times 8 & 13-4 & 8 \times 4\end{array}$ $\begin{array}{llllll}4 \times 4 & 13-4 & 11-4 & 8 \times 0 & 7 \times 4 & 8 \times 4\end{array}$ $\begin{array}{llllll}7 \times 4 & 7+4 & 8-4 & 5-3 & 8+4 & 8+4\end{array}$ $\begin{array}{llllll}4 \times 4 & 7-4 & 6+4 & 4 \times 7 & 7+4 & 8 \times 3\end{array}$ 

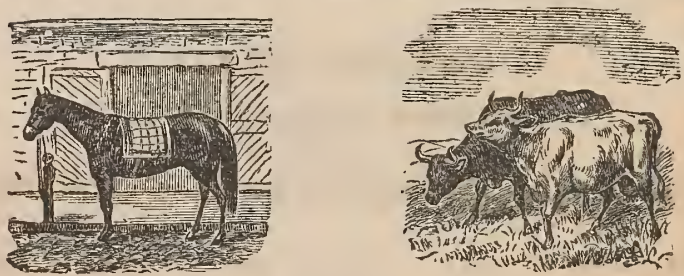

5 times 1 horse are 5 horses.

5 times 2 cows are 10 cows.
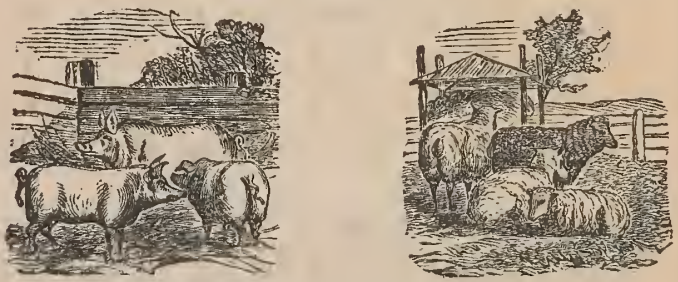

5 times 3 pigs are 15 pigs.

5 times 4 sheep are 20 sheep.

\section{LESSON XLVIII.}

Once 5 horses are 5 horses.

2 times 5 posts are 10 posts.

3 times 5 doors are 15 doors.

4 times 5 cows are 20 cows.

5 times 5 pigs are 25 pigs.

6 times 5 stys are 30 stys.

7 times 5 sheep are 35 sheep.

8 times 5 trees are 40 trees.

9 times 5 sheds are 45 sheds. 


\section{IES S N XIX.}

(See Manual, Sec. I., Exercise IV.)

Copy, complete, and read the following tables:

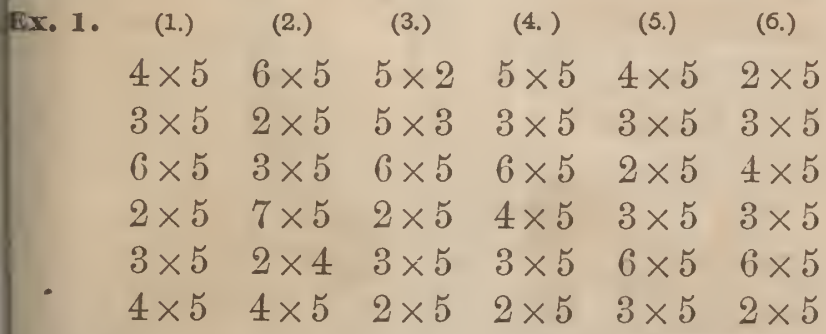

ix. 2. (1.)

(2.)

(3.)

(4.)

(5.)

(б.)

$\begin{array}{llllll}3 \times 5 & 6 \times 5 & 8 \times 5 & 9 \times 5 & 9 \times 5 & 3 \times 5\end{array}$ $\begin{array}{llllll}4 \times 5 & 7 \times 5 & 9 \times 5 & 7 \times 5 & 7 \times 5 & 5 \times 5\end{array}$ $\begin{array}{llllll}7 \times 5 & 3 \times 5 & 7 \times 5 & 8 \times 5 & 4 \times 5 & 9 \times 5\end{array}$ $\begin{array}{llllll}3 \times 5 & 0 \times 5 & 6 \times 5 & 5 \times 5 & 8 \times 5 & 7 \times 5\end{array}$ $\begin{array}{llllll}6 \times 5 & 7 \times 5 & 7 \times 5 & 7 \times 5 & 9 \times 5 & 6 \times 5\end{array}$ $\begin{array}{llllll}7 \times 5 & 6 \times 5 & 8 \times 5 & 8 \times 5 & 7 \times 5 & 3 \times 5\end{array}$

x. 3. (1.)

(2.)

(4.)

$\begin{array}{llllll}3+2 & 7-5 & 7+5 & 7-5 & 8+3 & 5-0\end{array}$ $\begin{array}{lllllll}7 \times 3 & 6 \times 5 & 12-5 & 8-5 & 5+3 & 5+0\end{array}$ $\begin{array}{lllllll}6+3 & 6+5 & 13-5 & 7-5 & 5+8 & 0+5\end{array}$ $\begin{array}{llllll}4-2 & 14-5 & 8+5 & 13-5 & 5-2 & 0 \times 5\end{array}$ $\begin{array}{lllllll}9-5 & 13-5 & 6+5 & 5-3 & 7+3 & 5 \times 5\end{array}$ $\begin{array}{llllll}9 \times 5 & 12-5 & 5-0 & 5+3 & 8-5 & 7-5\end{array}$ 


\section{4 \\ MULTIPLICATION.}

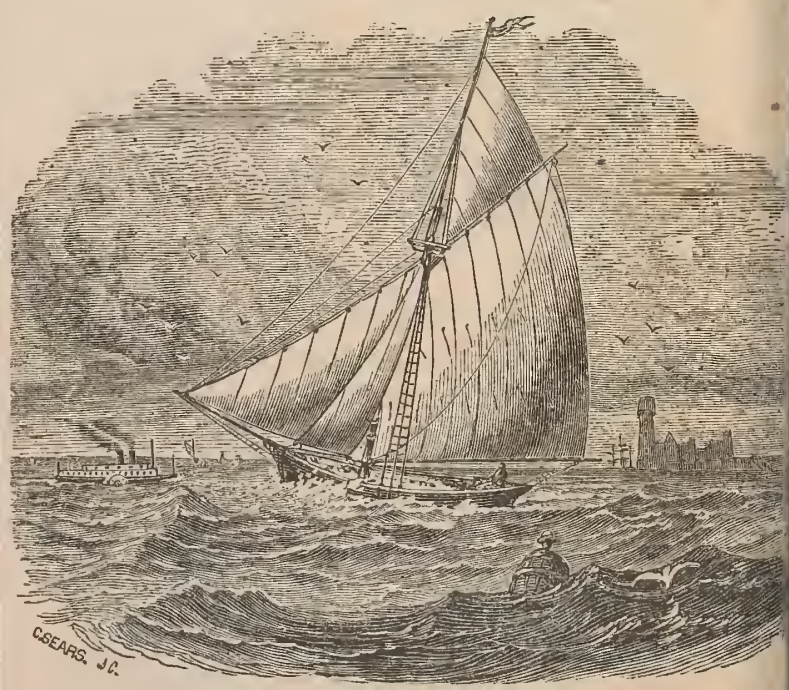

\section{LESSON L.}

Once 6 sloops are 6 sloops.

2 times 6 steamboats are 12 steamboats.

3 times 6 towers are 18 towers.

4 times 6 houses are 24 houses.

5 times 6 men are 30 men.

6 times 6 birds are 36 birds.

7 times 6 men are 42 men.

8 times 6 masts are 48 masts.

9 times 6 flags are 54 flags.

ProBleus. - If a ship have 3 masts, how many masts have $6 \mathrm{st}$ ships? There are 12 sea-gulls in a flock; a sailor shot 5 of the how many escaped? 


\section{IES SON II.}

(See Mannal, Sec. I., Exercise IV.)

Copy, complete, and read the following tables:

Ax. 1.

(2.)

(3)

(4.)

(5.)

(6.)

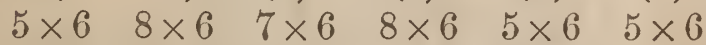
$\begin{array}{llllll}4 \times 6 & 7 \times 6 & 9 \times 6 & 3 \times 6 & 3 \times 6 & 7 \times 6\end{array}$

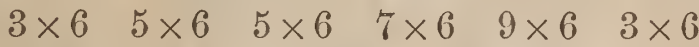
$\begin{array}{llllll}4 \times 6 & 3 \times 6 & 8 \times 6 & 3 \times 6 & 3 \times 6 & 6 \times 0\end{array}$ $\begin{array}{llllll}3 \times 6 & 2 \times 6 & 9 \times 6 & 9 \times 6 & 3 \times 6 & 3 \times 6\end{array}$ $\begin{array}{llllll}2 \times 6 & 7 \times 2 & 3 \times 6 & 8 \times 6 & 9 \times 5 & 9 \times 6\end{array}$
4x. 2. (1.)
(2.)
(3.)
(4.)
(5.)
(6.)

$2+3 \quad 4 \times 2 \quad 4+6 \quad 5+3 \quad 4+6 \quad 13-3$ $6+4 \quad 3 \times 6 \quad 6+6 \quad 6+2 \quad 3+6 \quad 11-6$ $\begin{array}{llllll}4 \times 6 & 5+6 & 4 \times 6 & 4-3 & 6+3 & 12-6\end{array}$ $\begin{array}{llllll}3+6 & 6-5 & 3+6 & 6-6 & 6-3 & 4+6\end{array}$ $6-313-6 \quad 8-6 \quad 6+6 \quad 5 \times 6 \quad 12-6$ $2+62 \times 6 \quad 13-6 \quad 6 \times 3 \quad 4+6 \quad 4 \times 6$

3.
(1.)
(2.)
(3.)
(4.)
(5.)

(6.)

$\begin{array}{llllll}5+6 & 3+6 & 4+6 & 7 \times 0 & 5+6 & 15-6\end{array}$ $\begin{array}{llllll}5+3 & 6-3 & 4-2 & 6 \times 0 & 6 \times 6 & 13-6\end{array}$ $6+3 \quad 7+4 \quad 4 \times 6 \quad 6-0 \quad 6 \times 5 \quad 10-6$ $2+6 \quad 7 \times 3 \quad 3 \times 6 \quad 6+0 \quad 6-5 \quad 16-6$ $\begin{array}{llllll}6-2 & 7 \times 6 & 7-6 & 0+6 & 7+6 & 11-6\end{array}$ $\begin{array}{llllll}6-0 & 7+6 & 7-0 & 0 \times 6 & 6+9 & 6+5\end{array}$ 


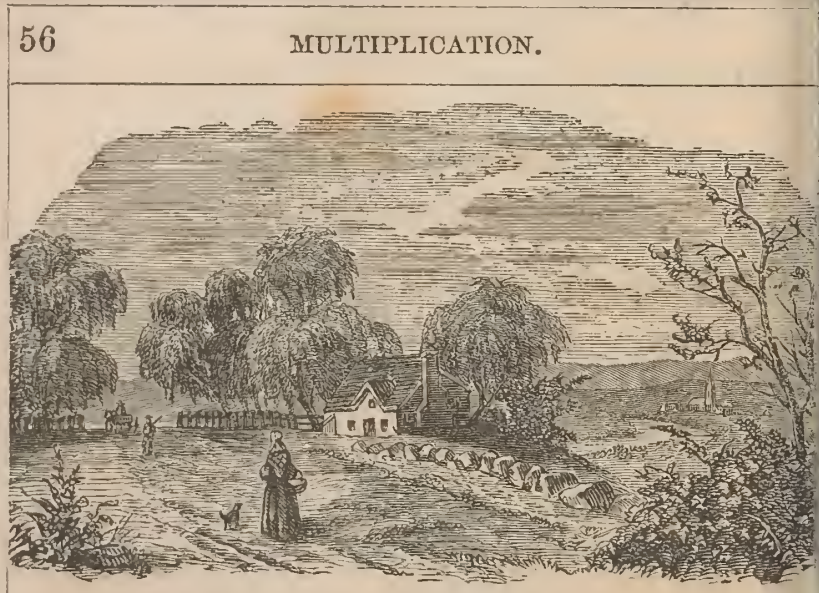

LESSON LII.

Once 7 houses are 7 houses.

2 times 7 windows are 14 windows.

3 times 7 doors are 21 doors.

4 times 7 carts are 28 carts.

5 times 7 men are 35 men.

6 times 7 women are 42 women.

7 times 7 trees are 49 trees.

8 times 7 churches are 56 churches.

9 times 7 dogs are 63 dogs.

Problems. - If the house has 3 windows, how many windows wil 4 such houses have? The man can carry 7 bushels of apples in hi: cart, how many bushels can he carry in going 5 times? The womar went to market $\zeta$ times, and each time carried 7 eggs in her basket how many eggs did she carry to market? There are 13 large stone. by the side of the road; if 6 of them should be carried away, hov many would remain? On a rose-bush there are 5 roses; how man: roses on 7 such bushes? 


\section{LES SON IIII.}

(See Manual, Sec. I., Exercise IV.)

Copy, complete, and read the following tables:

Ex. 1.

(1.)

(2.)

(3.)

(4.)

(5.)

(6.)

$\begin{array}{llllll}3 \times 7 & 4 \times 7 & 2 \times 7 & 5 \times 7 & 6 \times 7 & 5 \times 7\end{array}$ $\begin{array}{lllllll}4 \times 7 & 6 \times 7 & 3 \times 7 & 0 \times 7 & 3 \times 7 & 6 \times 7\end{array}$ $\begin{array}{llllll}6 \times 7 & 3 \times 7 & 8 \times 7 & 5 \times 7 & 6 \times 7 & 4 \times 7\end{array}$ $\begin{array}{llllll}5 \times 7 & 8 \times 7 & 5 \times 7 & 8 \times 7 & 8 \times 7 & 6 \times 7\end{array}$ $\begin{array}{llllll}3 \times 7 & 6 \times 7 & 7 \times 7 & 8 \times 7 & 9 \times 7 & 3 \times 7\end{array}$ $7 \times 7 \quad 5 \times 7 \quad 4 \times 7 \quad 6 \times 7 \quad 3 \times 7 \quad 5 \times 7$

Ex. 2.

(1.)

(2.)

(3.)

(4.)

(5.)

(6.)

$\begin{array}{lllll}3 \times 7 & 2 \times 7 & 5 \times 7>3 \times 7 & 15-7 & 6+7\end{array}$ $\begin{array}{llllll}4 \times 7 & 14-7 & 6 \times 7 & 6 \times 7 & 8 \times 7 & 8 \times 7\end{array}$ $\begin{array}{llllll}12-7 & 6 \times 7 & 8 \times 7 & 1+7 & 6+7 & 5+7\end{array}$ $\begin{array}{llllll}3 \times 7 & 8 \times 7 & 9-7 & 8-7 & 9 \times 7 & 4 \times 7\end{array}$ $\begin{array}{llllll}6+7 & 15-7 & 6+7 & 0 \times 7 & 6+7 & 8 \times 7\end{array}$ $\begin{array}{llllll}5+7 & 4 \times 7 & 8 \times 7 & 6 \times 7 & 9 \times 7 & 9-7\end{array}$

Ex. 3.

(1.)

(2.)

(3.)

(4.)

(5.)

(6.)

$\begin{array}{llllll}3 \times 1 & 3 \times 3 & 6+3 & 15-7 & 8 \times 5 & 4+3\end{array}$ $\begin{array}{llllll}3+7 & 4-3 & 7-3 & 7+8 & 6 \times 7 & 7 \times 7\end{array}$ $4 \times 3 \quad 8+616-7 \quad 16-8 \quad 7 \times 6 \quad 7-7$ $7-2 \quad 4 \times 3 \quad 13-7 \quad 4 \times 3 \quad 9-7 \quad 3-3$ $7+35 \times 7 \quad 16-7 \quad 9+6 \quad 8-6 \quad 8-8$ $\begin{array}{llllll}6 \times 7 & 2-0 & 10-7 & 16-7 & 9+7 & 4-4\end{array}$ 


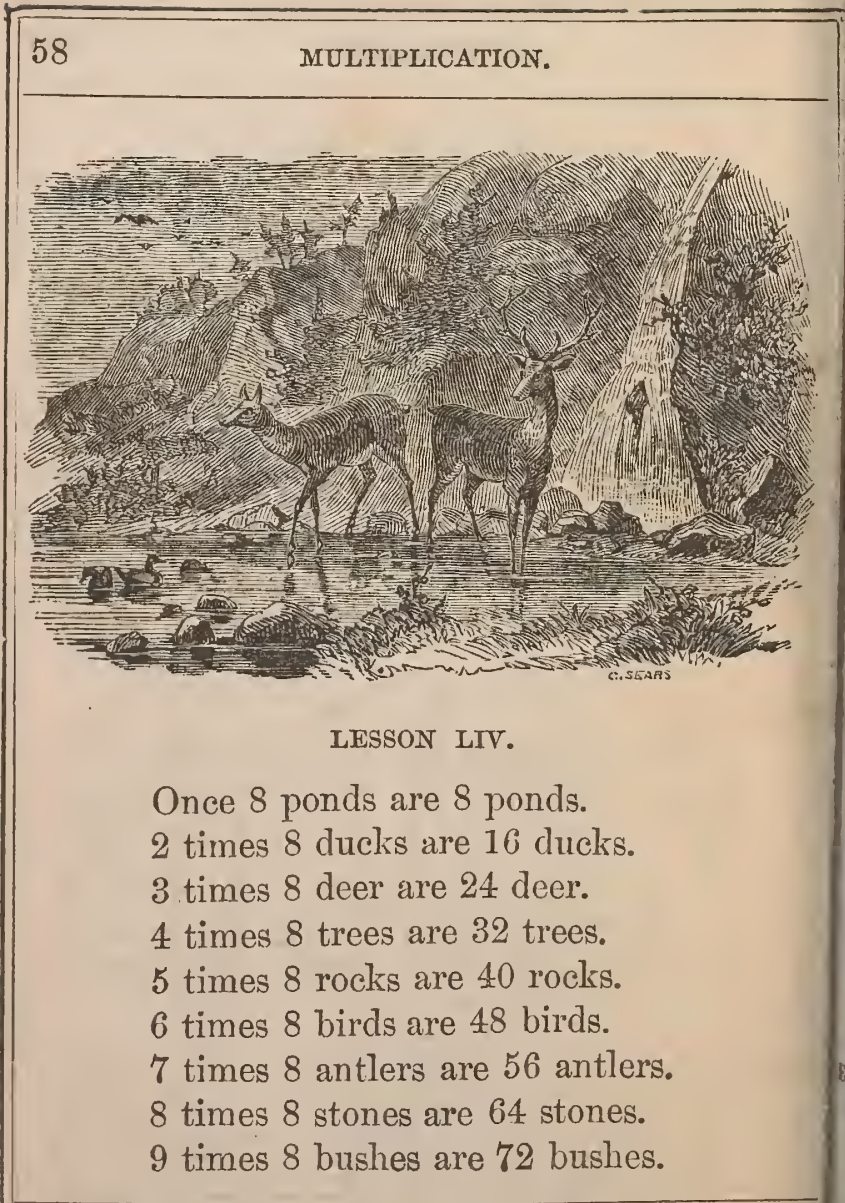

Pronicus.--Since 1 deer has 4 feet, how many feet have 2 deer Each duck has two wings; how many wings have 2 ducks? Eacl bird has 2 wings; how many wings have 8 birds? If a hunte should hoot 3 of the birds, how many would escape? 


\section{LESSON LV.}

(See Nanual, Sec I., Exerciso IV.)

Copy, complete, and read the following tables:

Ex. 1.

\author{
(1.)
}

(2.)

(3.)

(4.)

(5)

(6.)

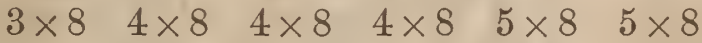
$\begin{array}{llllll}4 \times 8 & 3 \times 8 & 3 \times 8 & 3 \times 8 & 3 \times 8 & 6 \times 8\end{array}$ $\begin{array}{llllll}3 \times 8 & 7 \times 8 & 6 \times 8 & 6 \times 8 & 7 \times 8 & 7 \times 8\end{array}$ $\begin{array}{llllll}2 \times 7 & 3 \times 8 & 3 \times 8 & 3 \times 8 & 6 \times 8 & 4 \times 8\end{array}$ $\begin{array}{llllll}5 \times 8 & 6 \times 8 & 7 \times 8 & 7 \times 8 & 3 \times 8 & 3 \times 8\end{array}$ $\begin{array}{llllll}6 \times 8 & 4 \times 8 & 6 \times 8 & 6 \times 8 & 4 \times 8 & 6 \times 8\end{array}$

Ex. 2. (1.)

(4.)

(5.)

(6.)

$\begin{array}{llllll}3 \times 8 & 2 \times 8 & 2 \times 8 & 4 \times 8 & 5 \times 8 & 3 \times 8\end{array}$ $\begin{array}{llllll}7 \times 8 & 1 \times 8 & 4 \times 8 & 7 \times 8 & 6 \times 8 & 7 \times 8\end{array}$ $\begin{array}{llllll}2 \times 8 & 7 \times 8 & 7 \times 8 & 6 \times 8 & 7 \times 8 & 6 \times 8\end{array}$ $\begin{array}{llllll}3 \times 8 & 9 \times 8 & 6 \times 8 & 5 \times 8 & 6 \times 8 & 3 \times 8\end{array}$ $\begin{array}{llllll}7 \times 8 & 6 \times 8 & 3 \times 8 & 7 \times 8 & 7 \times 8 & 7 \times 8\end{array}$ $\begin{array}{llllll}3 \times 8 & 5 \times 8 & 1 \times 8 & 6 \times 8 & 5 \times 8 & 6 \times 8\end{array}$

Ex. 3.

(1.)

(2.)

(3.)

(4.)

(5.)

(6.)

$\begin{array}{llllll}4+8 & 17-8 & 5 \times 7 & 7-6 & 7-4 & 8-8\end{array}$ $\begin{array}{llllll}3+8 & 11-8 & 7 \times 8 & 7+4 & 15-6 & 8 \times 0\end{array}$ $\begin{array}{llllll}8-3 & 3+9 & 8-8 & 5+9 & 7 \times 4 & 0 \times 8\end{array}$ $\begin{array}{llllll}8-2 & 6+7 & 8 \times 8 & 9+5 & 8+6 & 8+0\end{array}$ $\begin{array}{lllll}13-9 & 4 \times 7 & 8+8 & 9 \times 8 \times 8 \times 6 & 0+8\end{array}$ $\begin{array}{llllll}15-8 & 7 \times 3 & 7 \times 8 & 7+8 & 8-6 & 8-0\end{array}$ 


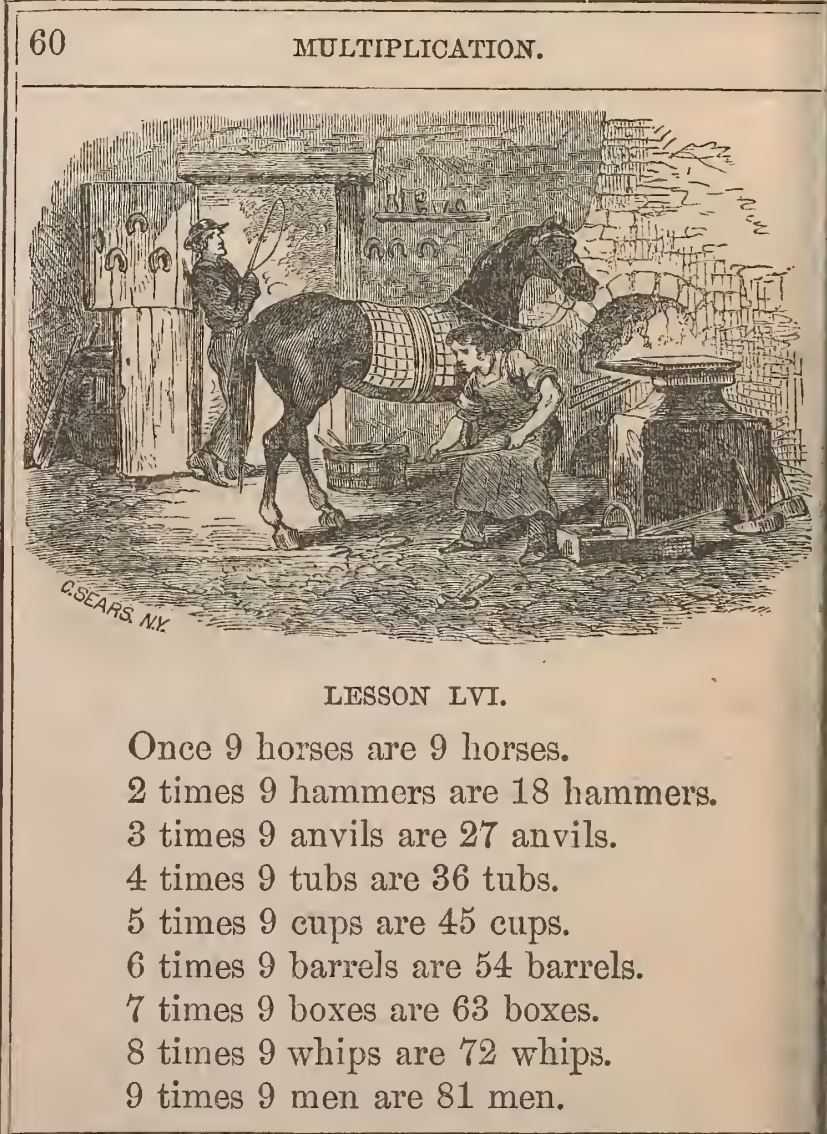

Problems.-One horse has 4 feet, how many feet hare 9 hor'ses: A man can shoe 5 horses in a day, how many horses can he shoe it 8 days? If one horse-shoe requires 6 nails, how many nails shoulc be driven in 2 horse-shoes? In 4 horse-shoes? There are 3 horse shoes hanging on one side of the door and 3 on the other side, how many on both? 


\section{IESSON IVII.}

(See Manual, Sec. I., Exercise IV.)

Copy, complete, and read the following tables:

\section{Ex. 1.}

(2.)

(3.)

(4.)

(5.)

(6.)

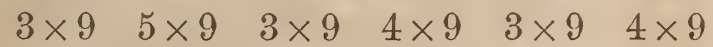

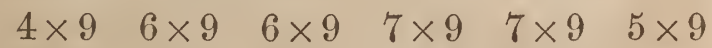

$\begin{array}{llllll}3 \times 9 & 7 \times 9 & 3 \times 9 & 3 \times 9 & 6 \times 9 & 7 \times 9\end{array}$

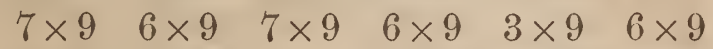

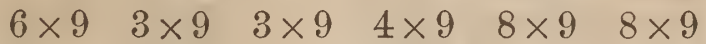

$5 \times 9 \quad 4 \times 9 \quad 0 \times 9 \quad 3 \times 9 \quad 3 \times 9 \quad 3 \times 9$

Ex. 2.

(1.) (2.)

(3.)

(4.)

(5.)

(6.)

$\begin{array}{llllll}3 \times 9 & 3 \times 3 & 5 \times 3 & 4+3 & 15-7 & 9-9\end{array}$

$4+6 \quad 4-3 \quad 7-3 \quad 9-7 \quad 16-8 \quad 9+0$

$4 \times 7 \quad 4+618-9 \quad 7 \times 9 \quad 10-9 \quad 9 \times 0$

$3-2 \quad 8-213-7 \times 8 \times 6 \quad 7-3 \quad 0+9$

$7-213-915-6 \quad 6-6 \quad 7+3 \quad 0 \times 9$

$\begin{array}{llllll}9-8 & 6-6 & 9-8 & 4+8 & 4+3 & 9-0\end{array}$

Ex. 3.

(1.) (2.)

(3.)

(4.)

(5.)

(6.)

$\begin{array}{llllll}3 \times 7 & 3 \times 7 & 6 \times 7 & 4+7 & 8+6 & 8+3\end{array}$ $\begin{array}{llllll}4 \times 7 & 8-4 & 5 \times 7 & 8+6 & 3+7 & 7+3\end{array}$ $\begin{array}{llllll}3+7 & 7-7 & 3+4 & 8 \times 7 & 9+3 & 8 \times 7\end{array}$ $\begin{array}{llllll}9 \times 9 & 8+3 & 6+9 & 9+7 & 9 \times 3 & 9 \times 7\end{array}$ $\begin{array}{llllll}8+7 & 7+7 & 6-1 & 9 \times 7 & 9-3 & 6-4\end{array}$ $\begin{array}{llllll}6 \times 9 & 4 \times 8 & 8+7 & 8+3 & 4+6 & 3+4\end{array}$ 


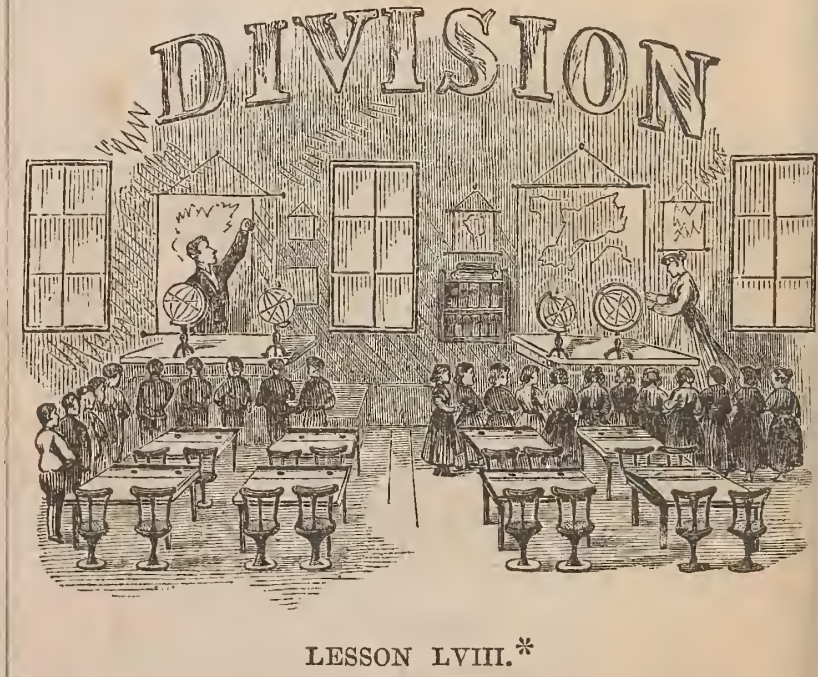

How many times can 2 tables be taken from 2 tables? How many times can 2 globes be taken from 4 globes? How many times are 2 maps contained in 6 maps? How many times are 2 desks contained in 8 desks? How many times are 2 boys contained in 10 boys? How many are half of 12 girls?

How many are one half of 14 books?

16 chairs are how many times 2 chairs?

18 window-panes are how many times 2 windowpanes?

* Note for the Teacher.-The teacher should not fail to show by means of objects, that 2 can be taken from a number as often as 2 is contained in it, or, that the two forms of expression are essentially the same. 


\section{LESSON IIX.*}

(See Manual, Sec. I., Exercise V.)

Copy, complete, and read the following tables: Ex. 1. Model.

(2.)

(3.)

(4.)

$$
\begin{array}{rrrrr}
4 \div 2=2 & 2 \div 2 & 2 \div 2 & 2 \div 2 & 4 \div 2 \\
8 \div 2=4 & 4 \div 2 & 8 \div 2 & 4 \div 2 & 16 \div 2 \\
6 \div 2=3 & 8 \div 2 & 4 \div 2 & 12 \div 2 & 14 \div 2 \\
2 \div 2=1 & 10 \div 2 & 12 \div 2 & 14 \div 2 & 12 \div 2 \\
8 \div 2=4 & 8 \div 2 & 10 \div 2 & 10 \div 2 & 16 \div 2 \\
2 \div 2=1 & 4 \div 2 & 6 \div 2 & 14 \div 2 & 8 \div 2
\end{array}
$$

Ex. 2. Model
(1.)

(2.)

(3.)

$16 \div 2=8 \quad 12 \div 2 \quad 10 \div 2 \quad 16 \div 2 \quad 4 \div 2$

$18 \div 2=9 \quad 16 \div 2 \quad 8 \div 2 \quad 12 \div 2 \quad 8 \div 2$

$12 \div 2=6 \quad 18 \div 2 \quad 6 \div 2 \quad 10 \div 2 \quad 12 \div 2$

$10 \div 2=5 \quad 20 \div 2 \quad 8 \div 2 \quad 8 \div 2 \quad 14 \div 2$

$8 \div 2=4 \quad 18 \div 2 \quad 4 \div 2 \quad 18 \div 2 \quad 4 \div 2$

$6 \div 2=3 \quad 16 \div 2 \quad 16 \div 2 \quad 12 \div 2 \quad 8 \div 2$

Ex. 3.
(1.)
(2.)

(3.)

(4.)

(5.)

$16 \div 2 \quad 8-2 \quad 7-2 \quad 5-2 \quad 5-2 \quad 4-2$ $16-8 \quad 8+2 \quad 16 \div 2 \quad 2-2 \quad 6 \div 2 \quad 6 \div 2$

$8+8 \quad 8 \div 2 \quad 14 \div 2 \quad 8+2 \quad 7+2 \quad 8-2$ $7-6 \quad 8 \times 2 \quad 6 \times 2 \quad 10 \div 2 \quad 2+8 \quad 18 \div 2$ $\begin{array}{llllll}7+2 & 9-2 & 2+8 & 7-2 & 2 \times 8 & 7-2\end{array}$ $12-9 \quad 6-2 \quad 9-2 \quad 4 \div 2 \quad 8 \div 2 \quad 12-2$

* Note for the Teacher.-Illustrate the meaning and the use of the sign of Division $(\div)$ in the following Exercises. 


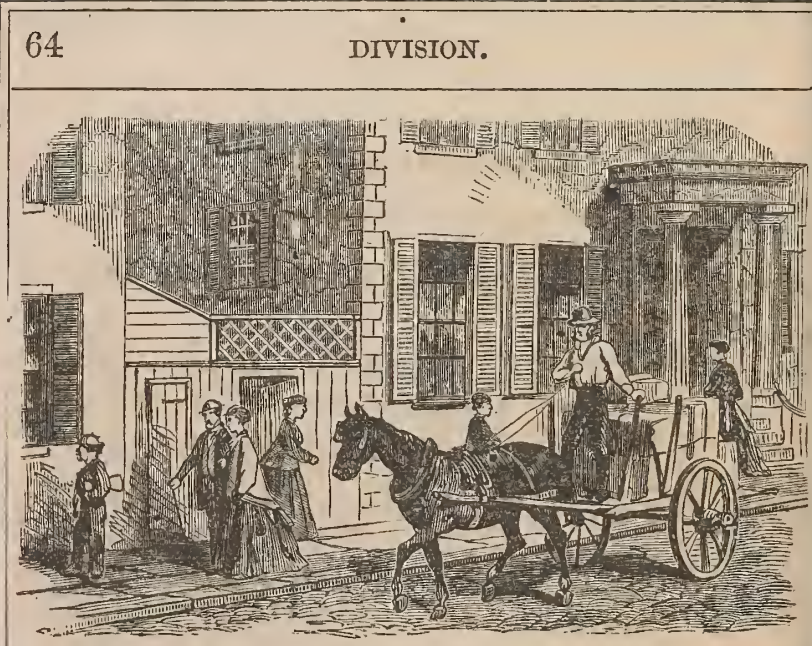

\section{LESSON LX.}

3 carts are once 3 carts.

6 horses are 2 times 3 horses.

9 boxes are 3 times 3 boxes.

12 pillars are 4 times 3 pillars.

15 windows are 5 times 3 windows.

18 men are 6 times 3 men.

21 doors are 7 times 3 doors.

24 canes are 8 times 3 canes.

Problems. - A carman bad 27 boxes to take to the depot, how many loads will they make if he draws 3 boxes at a load? A boy has 15 cents, how many pencils can he buy if they cost 3 cents each? A lady has 12 dollars, and if silk is 3 dollars a yard, how many yards can she buy? 12 window-panes are how many times 8 window-panes? 


\section{LESSON LXI.}

(See Manual, Sec. I., Exercise V.)

Copy, read, and complete the following tables :
Ex. 1.
(1.)
(2.)
(3.)
(4.)
(5.)
(6.)
$6 \div 3 \quad 6 \div 3 \quad 12 \div 3 \quad 15 \div 3 \quad 15 \div 3 \quad 15 \div 3$
$12 \div 3 \quad 9 \div 3 \quad 15 \div 318 \div 3 \quad 21 \div 3 \quad 21 \div 3$
$9 \div 3 \quad 15 \div 3 \quad 18 \div 3 \quad 15 \div 3 \quad 24 \div 3 \quad 27 \div 3$
$3 \div 3 \quad 12 \div 3 \quad 6 \div 3 \quad 21 \div 3 \quad 18 \div 3 \quad 24 \div 3$
$9 \div 3 \quad 9 \div 3 \quad 9 \div 3 \quad 18 \div 3 \quad 15 \div 3 \quad 18 \div 3$
$6 \div 3 \quad 12 \div 3 \quad 3 \div 3 \quad 6 \div 3 \quad 12 \div 3 \quad 15 \div 3$

Gx. 2. (1.) (2.) (3.) (4.) (5.)

$27 \div 3 \quad 15 \div 3 \quad 18 \div 3 \quad 21 \div 3 \quad 18 \div 3 \quad 12 \div 3$

$24 \div 3 \quad 6 \div 3 \quad 21 \div 3 \quad 18 \div 3 \quad 21 \div 3 \quad 9 \div 3$

$15 \div 3 \quad 9 \div 3 \quad 24 \div 3 \quad 24 \div 3 \quad 27 \div 3 \quad 6 \div 3$

$21 \div 3 \quad 12 \div 3 \quad 27 \div 3 \quad 27 \div 3 \quad 18 \div 3 \quad 18 \div 3$

$27 \div 3 \quad 15 \div 3 \quad 15 \div 3 \quad 21 \div 3 \quad 27 \div 3 \quad 24 \div 3$

$24 \div 318 \div 324 \div 315 \div 312 \div 3 \quad 27 \div 3$

Ex. 3. (1) (2.) (3.) (4.) (5.)

$6+3 \quad 4 \times 3 \quad 8+3 \quad 12 \div 3 \quad 6 \div 3 \quad 8 \times 3$ $4-3 \quad 9 \times 3 \quad 9+3 \quad 9 \times 3 \quad 9 \div 3 \quad 3 \times 3$ $7+3 \quad 12 \div 3 \quad 11-3 \quad 7+3 \quad 11-3 \quad 11-3$ $\begin{array}{llllll}8 \times 3 & 6+3 & 8 \times 3 & 8-3 & 8 \times 3 & 24 \div 3\end{array}$ $24 \div 3 \quad 8-3 \quad 27 \div 3 \quad 9 \div 3 \cdot 9+3 \quad 8+3$ $\begin{array}{llllll}7+3 & 11-3 & 7+3 & 8 \times 3 & 7+3 & 3+9\end{array}$ 


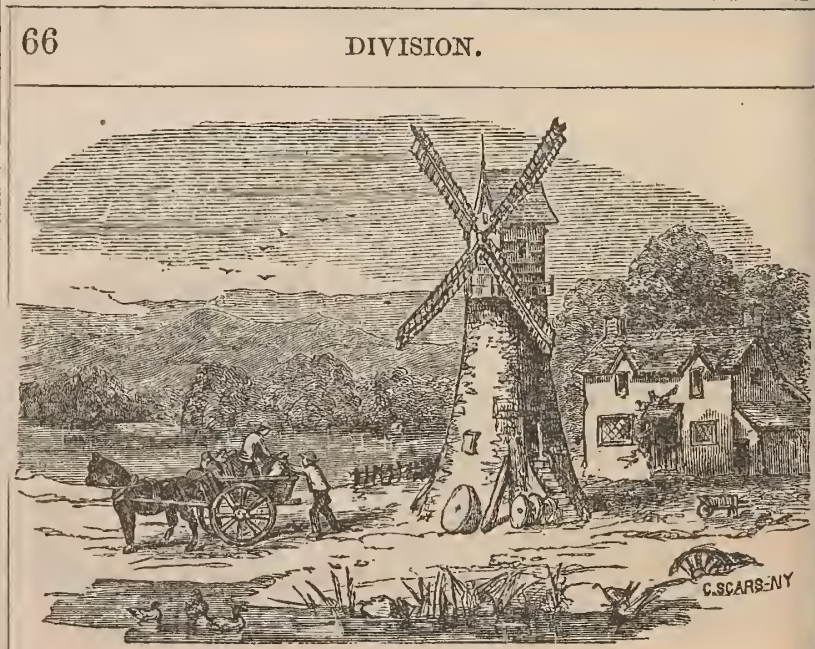

\section{LESSON LXII.}

4 birds are contained in 4 birds once. 4 spokes are contained in 8 spokes 2 times. 4 bags are contained in 12 bags 3 times. 4 mills are contained in 16 mills 4 times. 4 posts are contained in 20 posts 5 times. 4 houses are contained in 24 houses 6 times. 4 windows are contained in 28 windows 7 times. 4 horses are contained in 32 horses 8 times. 4 men are contained in 36 men 9 times.

Problems.-One wind-mill has 4 wings, how many wings will 4 such mills have? How many loads will 16 bags of corn make if a man draws 4 bags at a load? There are 4 windows in the house, how many windows will 6 such houses bave? There are 8 spokes in a wheel; how many spokes in 2 wheels? If a cart have 2 wheels, how many wheels have 4 carts? 


\section{LESSON LXIII.}

(See Manual, Sec. I., Exercise V.)

Copy, complete, and read the following tables:

Ex. 1.

(4.)

(5.)

(6.)

$4 \div 4 \quad 4 \div 4 \quad 8 \div 424 \div 428 \div 432 \div 4$ $8 \div 412 \div 412 \div 416 \div 432 \div 420 \div 4$ $12 \div 416 \div 416 \div 412 \div 428 \div 428 \div 4$ $8 \div 412 \div 420 \div 48 \div 424 \div 424 \div 4$ $12 \div 48 \div 412 \div 436 \div 416 \div 432 \div 4$ $4 \div 416 \div 4 \quad 8 \div 420 \div 412 \div 428 \div 4$

Ex. 2.

(1.)

(2.)

(3.)

(4.)

(5.)

(6.)

$32 \div 4 \quad 4 \div 4 \quad 8 \div 4 \quad 4 \div 4 \quad 28 \div 412 \div 4$ $24 \div 4 \quad 8 \div 412 \div 4 \quad 8 \div 420 \div 420 \div 4$ $12 \div 424 \div 432 \div 416 \div 416 \div 428 \div 4$ $8 \div 436 \div 436 \div 424 \div 420 \div 432 \div 4$ $20 \div 428 \div 424 \div 428 \div 428 \div 48 \div 4$ $16 \div 420 \div 412 \div 436 \div 436 \div 4 \quad 4 \div 4$

Ex. 3.

(1.)

(2.)

(3.)

(4.)

(5.)

$24 \div 4 \quad 32 \div 4 \quad 6 \times 4 \quad 8-4 \quad 5 \times 4 \quad 4 \times 8$ $\begin{array}{llllll}8 \times 4 & 8 \times 4 & 7-4 & 7 \times 4 & 7-4 & 9+4\end{array}$ $12-4 \quad 6-4 \quad 8 \times 4 \quad 36 \div 4 \quad 8 \times 4 \quad 9-4$ $6+4 \quad 8+4 \quad 7+4 \quad 7-4 \quad 4 \times 8 \quad 32 \div 4$ $8 \div 4 \quad 13-4 \quad 13-4 \quad 12-4 \quad 9 \times 4 \quad 7 \times 4$ $7+4 \quad 7 \times 4 \quad 7 \times 4 \quad 6+4 \quad 36 \div 4 \quad 6-4$ 


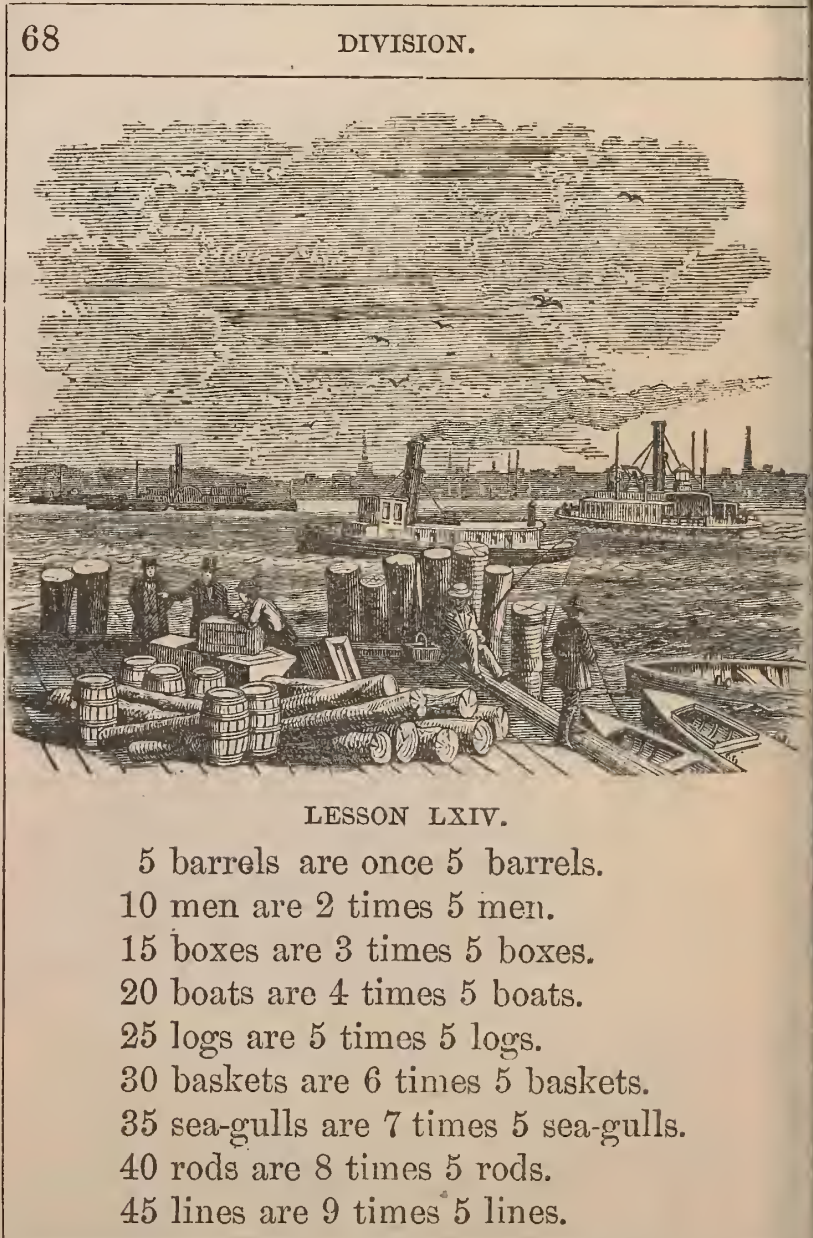

Problems.-There were 17 piles lying on the dock; 9 have been driven; how many remain? 
divis: $10 \%$

Division.

69

\section{IESSON LXV.}

(See Manual, Sec. I., Exercise V.)

Copy, read, and complete the following tables:

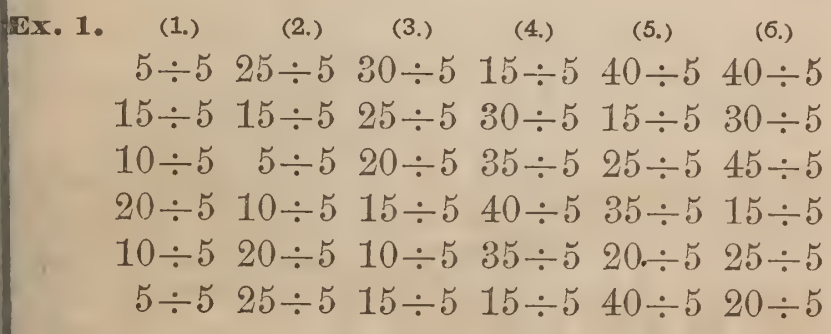

Ex. 2.

(1.) (2.)

(3.)

(4.),

(5.)

(6.)

$25 \div 515 \div 5 \quad 20 \div 5 \quad 5 \div 5 \quad 10 \div 515 \div 5$ $30 \div 510 \div 5 \quad 35 \div 515 \div 5 \quad 20 \div 5 \quad 25 \div 5$ $40 \div 5 \quad 5 \div 5 \quad 40 \div 525 \div 5 \quad 30 \div 530 \div 5$ $20 \div 5 \quad 30 \div 5 \quad 25 \div 535 \div 540 \div 540 \div 5$ $45 \div 540 \div 5 \quad 30 \div 545 \div 515 \div 545 \div 5$ $10 \div 545 \div 520 \div 525 \div 520 \div 5 \quad 35 \div 5$

2x.3. (1.)

(2.)

(3.)

(4.)

(5.)

(6.)

$25 \div 5 \quad 30 \div 5 \quad 7+545 \div 5 \quad 6+5 \quad 4 \times 5$

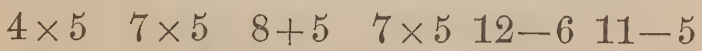
$3+5 \quad 5-513-5 \quad 5 \times 5 \quad 8+7 \quad 10 \div 5$ $8-5 \quad 8+5 \quad 40 \div 5 \quad 10-5 \quad 8-5 \quad 3 \times 5$

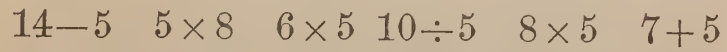
$10 \div 5 \quad 13-5 \quad 7-510 \times 5 \quad 6+5 \quad 9+5$ 


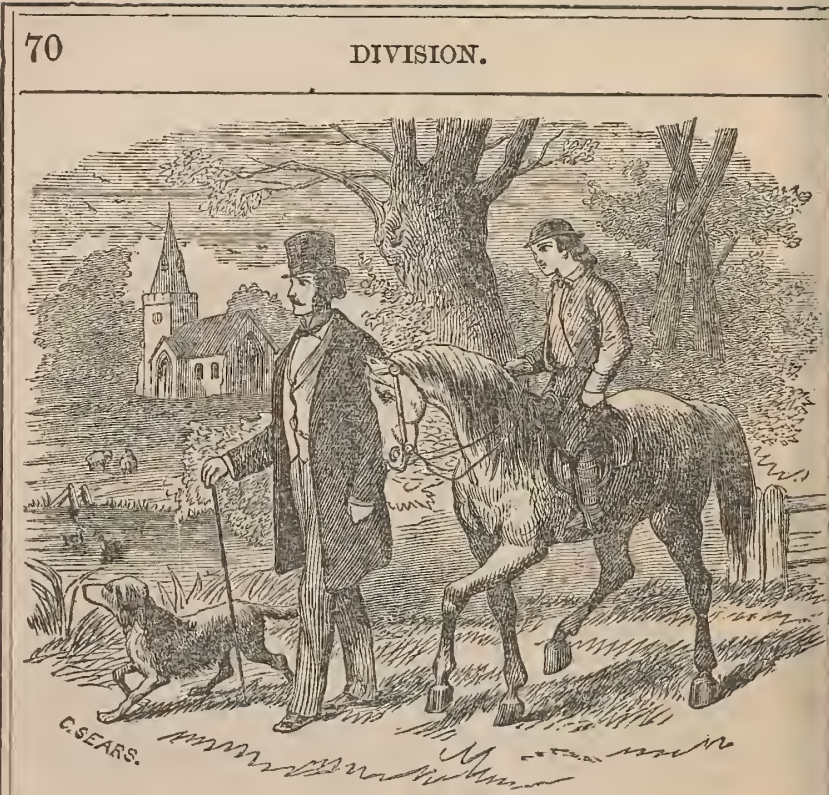

LESSON LXVI.

6 churches are once 6 churches.

12 sheep are 2 times 6 sheep.

18 ducks are 3 times 6 ducks.

24 dogs are 4 times 6 dogs.

30 men are 5 times 6 men.

36 boys are 6 times 6 boys.

42 saddles are 7 times 6 saddles.

48 bridles are 8 times 6 bridles.

54 horses are 9 times 6 horses.

Problems. - Willie rode 2 hours every day for a week, how many hours did be ride in all? 


\section{LESSON LXVII.}

(See Manual, Sec I., Exercise V.)

Copy, complete, and read the following tables:
Ex. 1
(1.)
(2.)
(3.)
(4.)
(5)
$6 \div 630 \div 630 \div 642 \div 648 \div 654 \div 6$ $18 \div 618 \div 636 \div 612 \div 630 \div 648 \div 6$ $12 \div 6 \quad 6 \div 6 \quad 24 \div 6 \quad 6 \div 6 \quad 36 \div 6 \quad 36 \div 6$ $24 \div 618 \div 618 \div 636 \div 648 \div 642 \div 6$ $6 \div 630 \div 612 \div 642 \div 624 \div 624 \div 6$ $18 \div 624 \div 6 \quad 6 \div 618 \div 618 \div 654 \div 6$

Ex. 2. $\begin{array}{cccccc}\text { (1.) } & \text { (2.) } & \text { (3.) } & \text { (4.) } & \text { (5.) } & \text { (6.) } \\ 42 \div 6 & 12 \div 6 & 24 \div 6 & 30 \div 6 & 42 \div 6 & 18 \div 6 \\ 48 \div 6 & 18 \div 6 & 30 \div 6 & 36 \div 6 & 30 \div 6 & 24 \div 6 \\ 30 \div 6 & 30 \div 6 & 42 \div 6 & 42 \div 6 & 36 \div 6 & 36 \div 6 \\ 12 \div 6 & 42 \div 6 & 48 \div 6 & 54 \div 6 & 48 \div 6 & 48 \div 6 \\ 54 \div 6 & 54 \div 6 & 18 \div 6 & 6 \div 6 & 18 \div 6 & 42 \div 6 \\ 30 \div 6 & 18 \div 6 & 12 \div 6 & 18 \div 6 & 12 \div 6 & 54 \div 6\end{array}$

9x. 3.

(1.)

(2.)

(3.)

(4.)

(5.)

(6.)

$24 \div 6 \quad 6 \times 8 \quad 7-6 \quad 5 \times 6 \quad 4 \times 6 \quad 13-6$ $24 \div 4 \quad 5 \times 6 \quad 5 \times 6 \quad 7-5 \quad 8 \times 8 \quad 7-6$ $2 \times 6 \quad 13-613-613-6 \quad 7-6 \quad 8 \times 6$

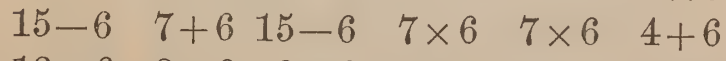
$13-6 \quad 8+6 \quad 8+6 \quad 12 \div 6 \quad 14-6 \quad 8 \times 6$ $8 \times 6 \quad 48 \div 6 \quad 8 \times 6 \quad 8-6 \quad 11-6 \quad 7 \times 6$ 


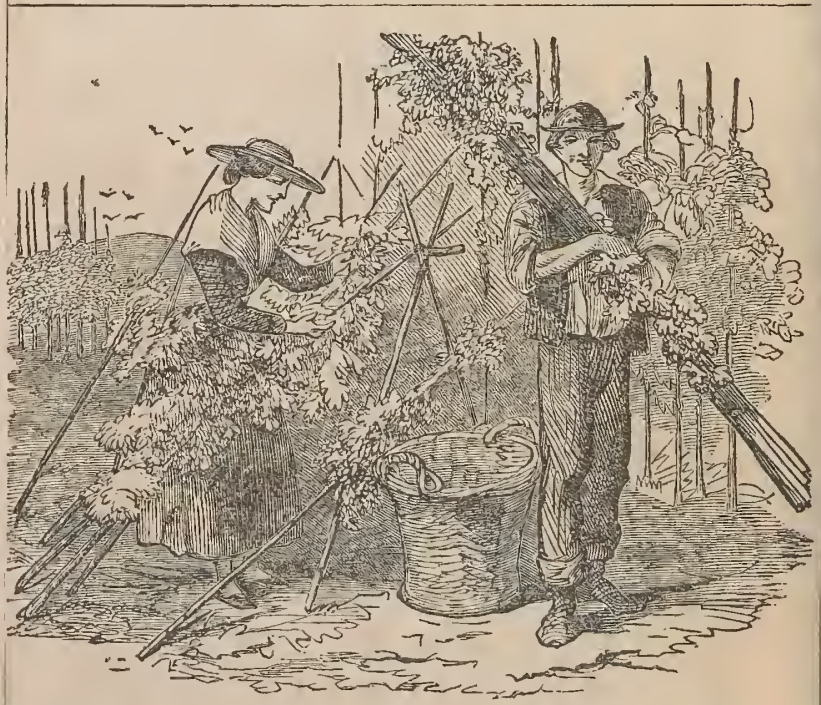

\section{LESSON IXVIII.}

7 baskets are once 7 baskets.

14 hop-poles are 2 times 7 hop-poles.

21 men are 3 times 7 men.

28 girls are 4 times 7 girls.

35 hop-vines are 5 times 7 hop-vines.

42 birds are 6 times 7 birds.

49 hats are 7 times 7 hats.

56 coats are 8 times 7 coats.

63 knives are 9 times 7 knives.

Problekns.-There are 8 birds flying away from the hop-yard; they are separated into 2 flocks; how many birds in each flock? 


\section{4 \\ DIVISION.}

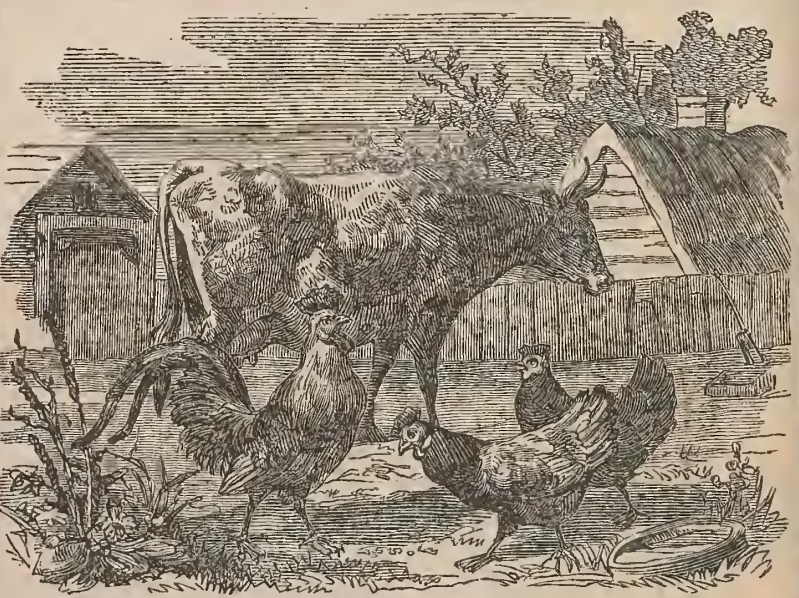

LESSON LIXX.

8 cows are once 8 cows.

16 hens are 2 times 8 hens.

24 houses are 3 times 8 houses.

32 feet are 4 times 8 feet.

40 claws are 5 times 8 claws.

48 dishes are 6 times 8 dishes.

56 boards are 7 times 8 boards.

64 doors are 8 times 8 doors.

72 windows are 9 times 8 windows.

Problems.-Henry found a nest containing $8 \mathrm{eggs}$; how many eggs will 2 such nests contain? The cow gives 8 quarts of milk every night and morning; how many quarts does she give each day? 


\section{LESSON LXXI.}

(See Manual, Sec. I., Exercise V.)

Copy, complete, and read the following tables:

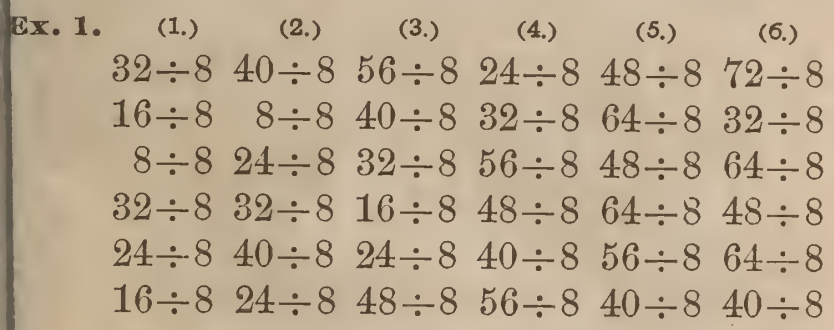

Ix. 2.

(1.)

(2.)

(3.)

(4.)

(5.)

(6.)

$40 \div 8 \quad 48 \div 856 \div 8 \quad 24 \div 8 \quad 24 \div 8 \quad 48 \div 8$ $48 \div 856 \div 8 \quad 72 \div 8 \quad 8 \div 8 \quad 56 \div 8 \quad 56 \div 8$ $72 \div 872 \div 8 \quad 56 \div 8 \quad 48 \div 8 \quad 40 \div 8 \quad 72 \div 8$ $32 \div 8 \quad 24 \div 8 \quad 40 \div 8 \quad 16 \div 8 \quad 48 \div 8 \quad 24 \div 8$ $48 \div 8 \quad 64 \div 8 \quad 48 \div 8 \quad 64 \div 8 \quad 32 \div 8 \quad 8 \div 8$ $32 \div 840 \div 8 \quad 64 \div 8 \quad 24 \div 8 \quad 24 \div 8 \quad 72 \div 8$

4x. 3.

(1.)

(2.)

(3.)

(4.)

(5.)

(6.)

$40 \div 832 \div 872 \div 840 \div 815-848 \div 8$ $6 \times 8 \quad 4 \times 8 \quad 64 \div 8 \quad 6 \times 8 \quad 48 \div 8 \quad 6+8$ $7+8 \quad 6 \times 8 \quad 7 \times 8 \quad 72 \div 8 \quad 6 \times 8 \quad 16-8$ $\begin{array}{llllll}16-8 & 8 \times 8 & 3 \times 8 & 9-8 & 15-8 & 11-8\end{array}$ $\begin{array}{llllll}17-8 & 13-8 & 15-8 & 7+8 & 9+8 & 3 \times 8\end{array}$ $\begin{array}{llllll}14-8 & 5+8 & 12-8 & 6 \times 8 & 6+8 & 8 \times 7\end{array}$ 


\section{6 \\ DIVISION.}

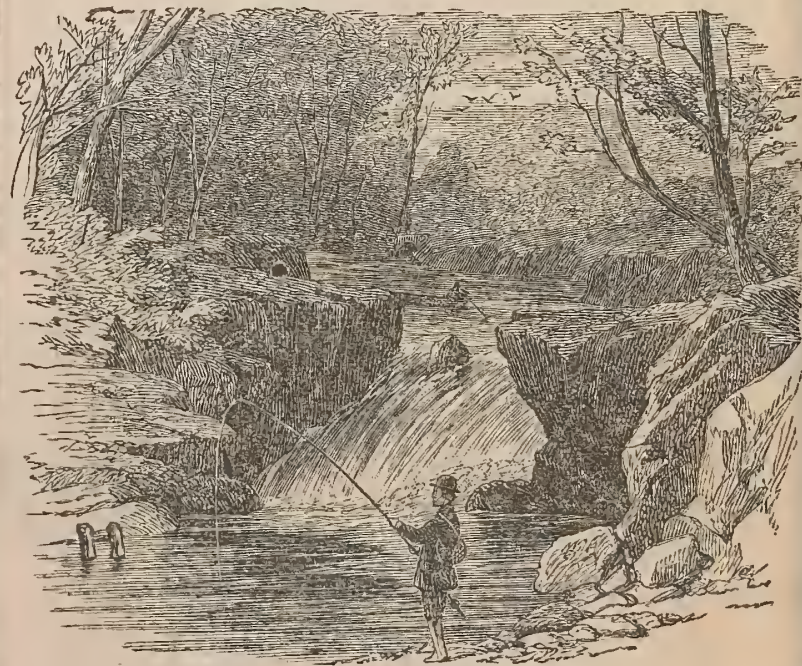

\section{LESSON LXXII.}

9 men are once 9 men.

18 boats are 2 times 9 boats.

27 poles are 3 times 9 poles.

36 bridges are 4 times 9 bridges.

45 trees are 5 times 9 trees.

54 rocks are 6 times 9 rocks.

63 birds are 7 times 9 birds.

72 fishes are 8 times 9 fishes.

81 fish-lines are 9 times 9 fish-lines.

Problems. $-\Lambda$ man caught 4 fishes the first hour and 7 the next hour, how many did he catch in the 2 hours? 


\section{IESSON IXXIII.}

(See Manual, Sec I., Exercise V.)

Copy, complete, and read the following tables:

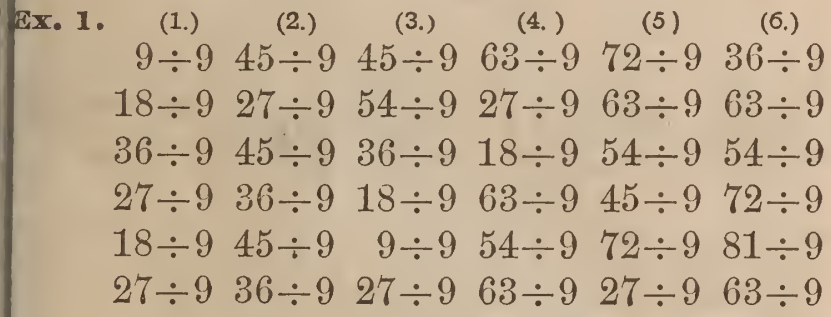

Ex. 2.

(1)

(2.)

(3.)

(4.)

(5.)

(6.)

$54 \div 945 \div 927 \div 918 \div 945 \div 918 \div 9$ $63 \div 954 \div 936 \div 927 \div 963 \div 9 \quad 9 \div 9$ $81 \div 963 \div 963 \div 9 \quad 9 \div 981 \div 927 \div 9$ $18 \div 981 \div 981 \div 945 \div 972 \div 963 \div 9$ $45 \div 936 \div 963 \div 927 \div 981 \div 936 \div 9$ $27 \div 963 \div 954 \div 981 \div 918 \div 945 \div 9$

Ex. 3. (1.)

(2.)

(2.) (3.)

(4.)

(5.)

$6 \times 8 \quad 5 \times 9 \quad 6+9 \quad 9+1 \quad 45 \div 963 \div 9$ $81 \div 972 \div 917-9 \quad 9 \times 9 \quad 5 \times 9 \quad 4 \times 9$ $8 \times 963 \div 936 \div 927 \div 945 \div 9 \quad 9+9$ $3+9 \quad 18-9 \quad 8 \times 9 \quad 10-9 \quad 5+8 \quad 9-9$ $9+6 \quad 9 \times 9 \quad 6+9 \quad 1+9 \quad 13-9 \quad 8 \times 9$ $17-97 \times 7 \quad 17-910+8 \quad 8 \times 818 \div 9$ 
$\rightarrow+2$

78

FRAC'TIONS.

FRACTIONS.

\section{L曰SSON IXXIV.}

$-\frac{2}{3} \cdot \frac{2}{8}$

(See Manual, Sec. I., Exercise VI.)
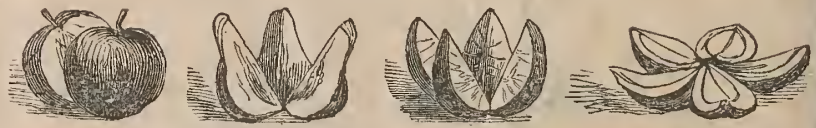

Two halves. Three thirds. Four fourths.

Five fifths.

How many halves in one apple?

How many halves in one peach?

How many thirds in one pie?

How many thirds in one fish?

How many fourths in one horse?

How many fourths in one loaf?

How many fifths in one string?

How many tifths in one stick?

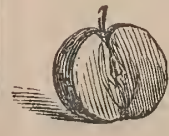

One half.

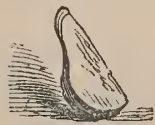

One third.

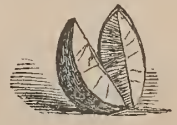

Two fourths.

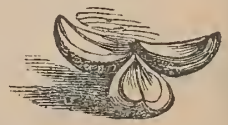

Three fifths.

How many halves make one melon?

How many thirds make one pear?

How many fourths make one orange?

How many fifths make one apple? 


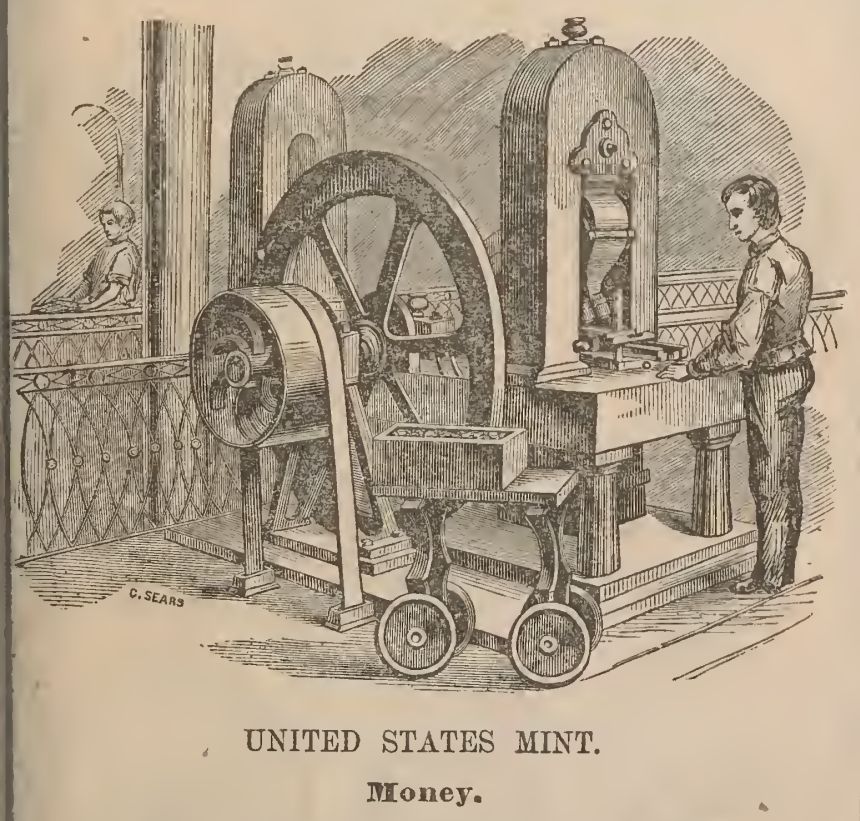

A mint is the place where money is made.

\section{Federal Money.}

Federal money is the currency of the United States.

\section{LESSON LXXV.}

(See Manual, Sec. I., Excreise VII.)

10 mills (m.) make 1 cent. ct.

10 cents make 1 dime. $d$.

10 dimes make 1 dollar. \$.

10 dollars make 1 eagle. E. 


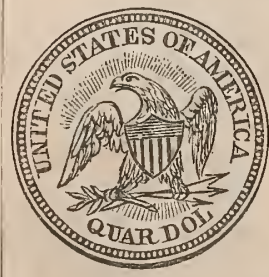

Silver.
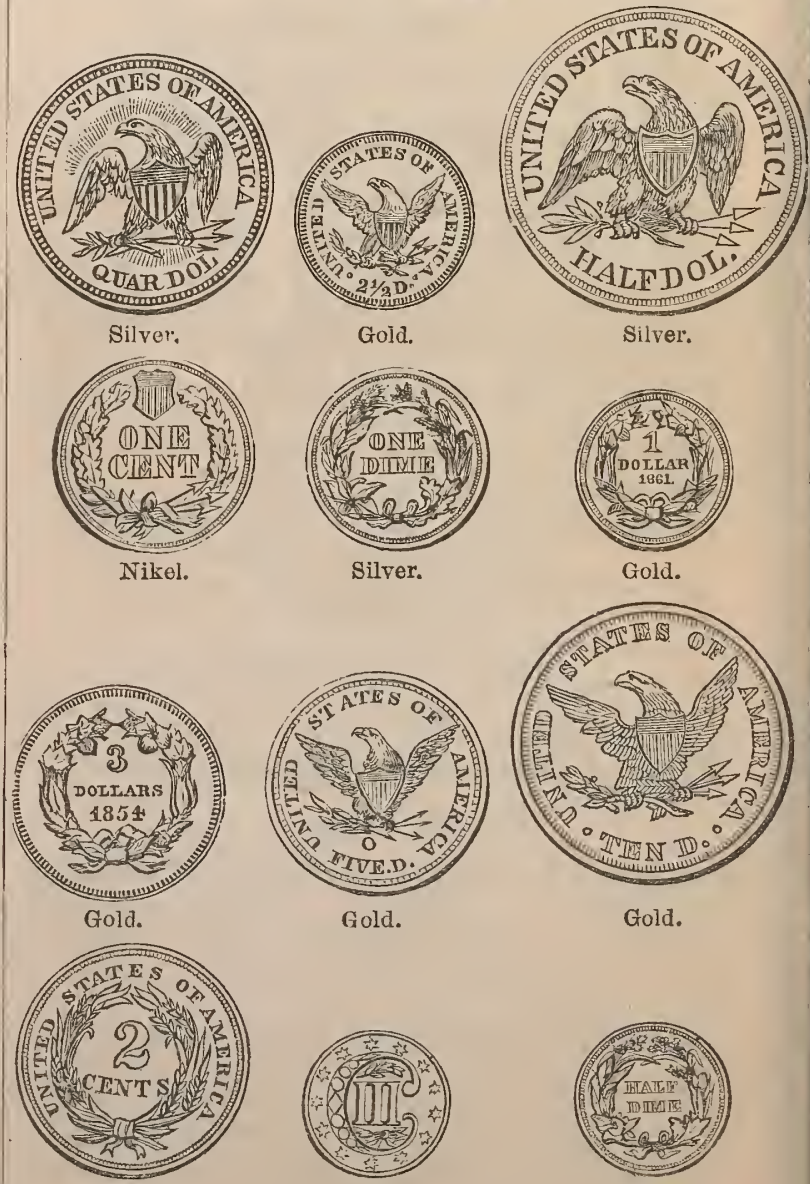

Copper.
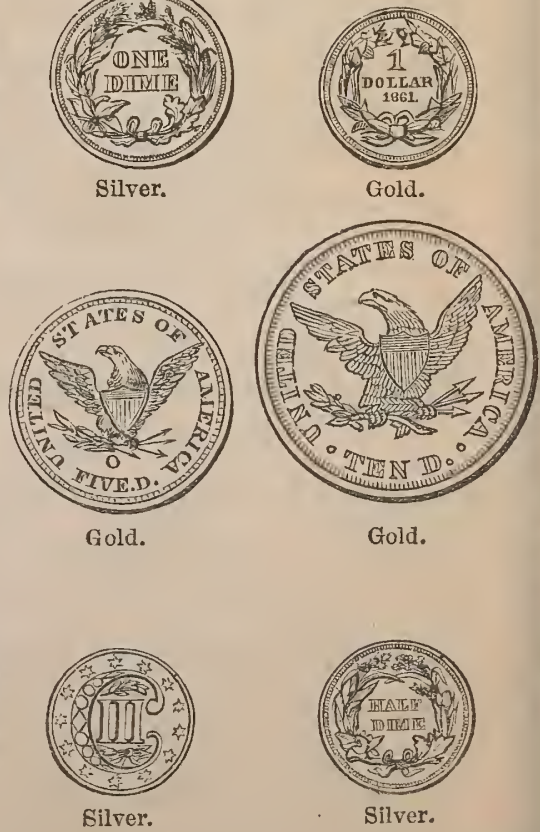


\section{LESSON LXXVI.}

(Seo Manual, Sec. I., Exerciso VII.)

\section{English PIoney.}

English money is the currency of Great Britain. TABLE.

4 farthings (far.) make 1 penny. $d$. 12 pence make 1 shilling. s. 20 shillings make $\left\{\begin{array}{c}1 \text { pound or } \\ \text { sovereign. }\end{array}\right\} £$.

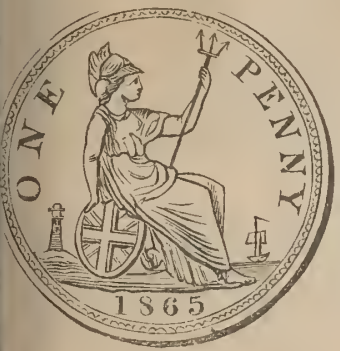

Copper-2 cts.

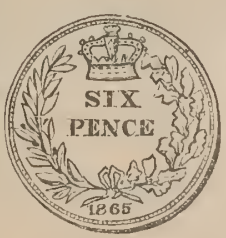

Silver-12 cts.

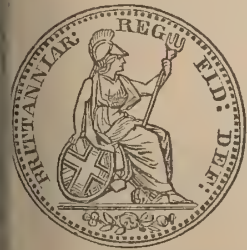

Far.-Copper -5 mills.

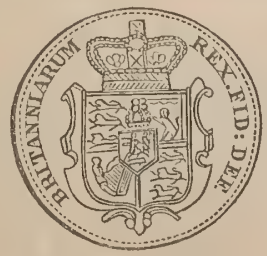

Sov.-Gold-\$4 84 .

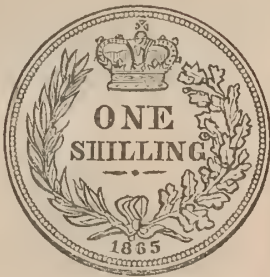

Silver -24 ets. Note.-The franc is a French coin.

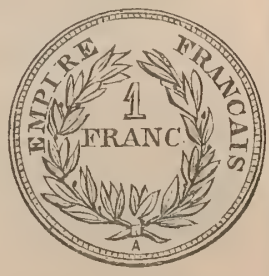

Silver-18 cents. 


\section{2 \\ ENGLISH MONEY.}

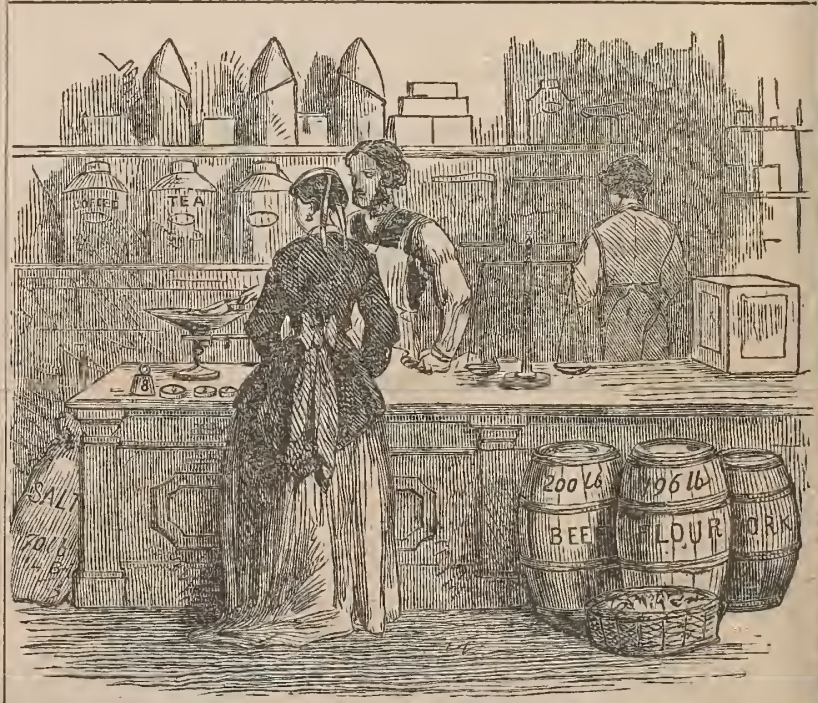

\section{Avolldupois Woight.}

Avoirdupois weight is used for weighing all common articles.

\section{LESSON LXXVI.}

(See Mranual, Sec. I., Exercise VII.)

16 drams (dr.) make

16 ounces make

25 pounds make

100 pounds or

4 quarters $\}$ make

20 hundred weight make 1 ton.
1 ounce. $\mathrm{oz}$.

1 pound. lb.

1 quarter, qr.

$1\left\{\begin{array}{c}\text { hundred } \\ \text { weight }\end{array}\right\}$ ervt.

T. 


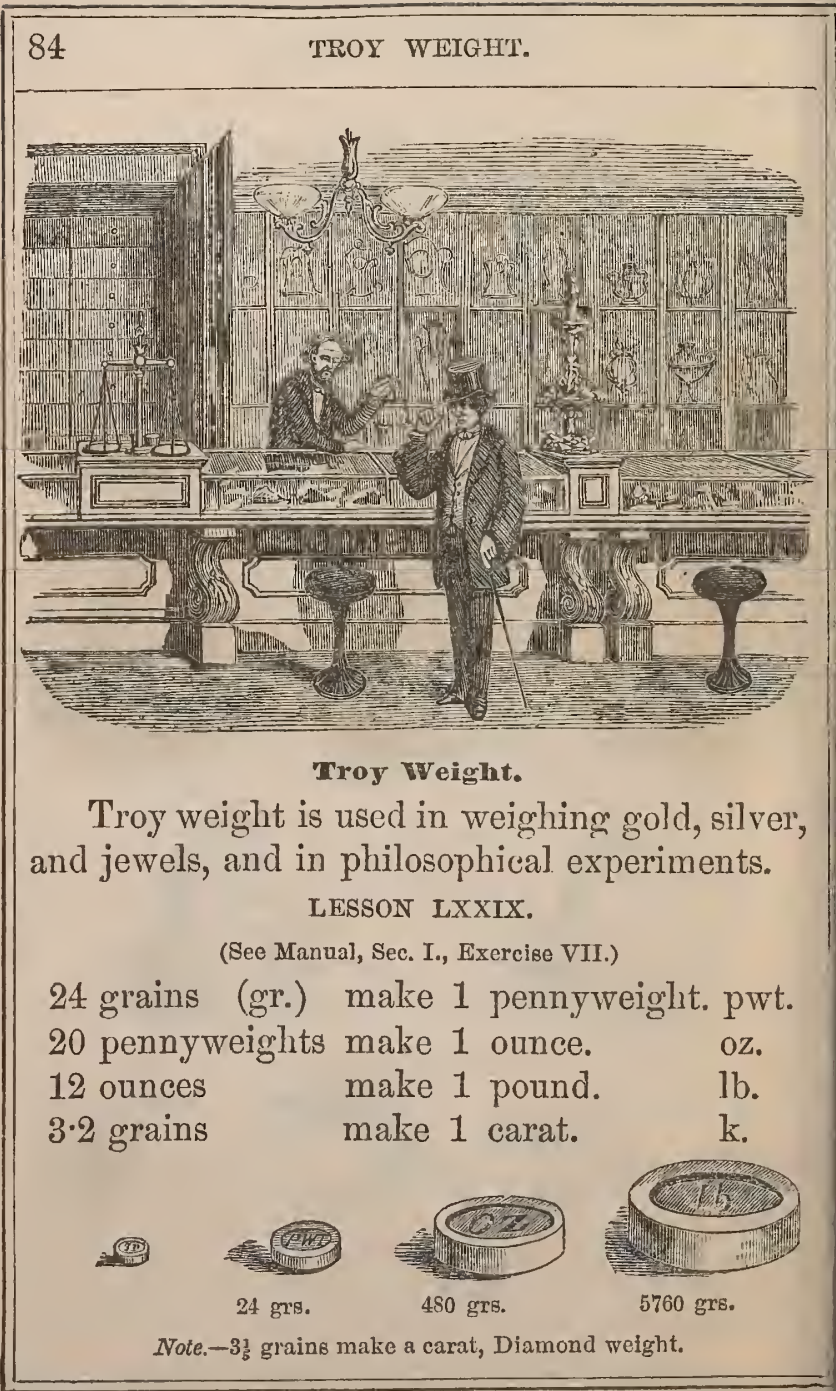




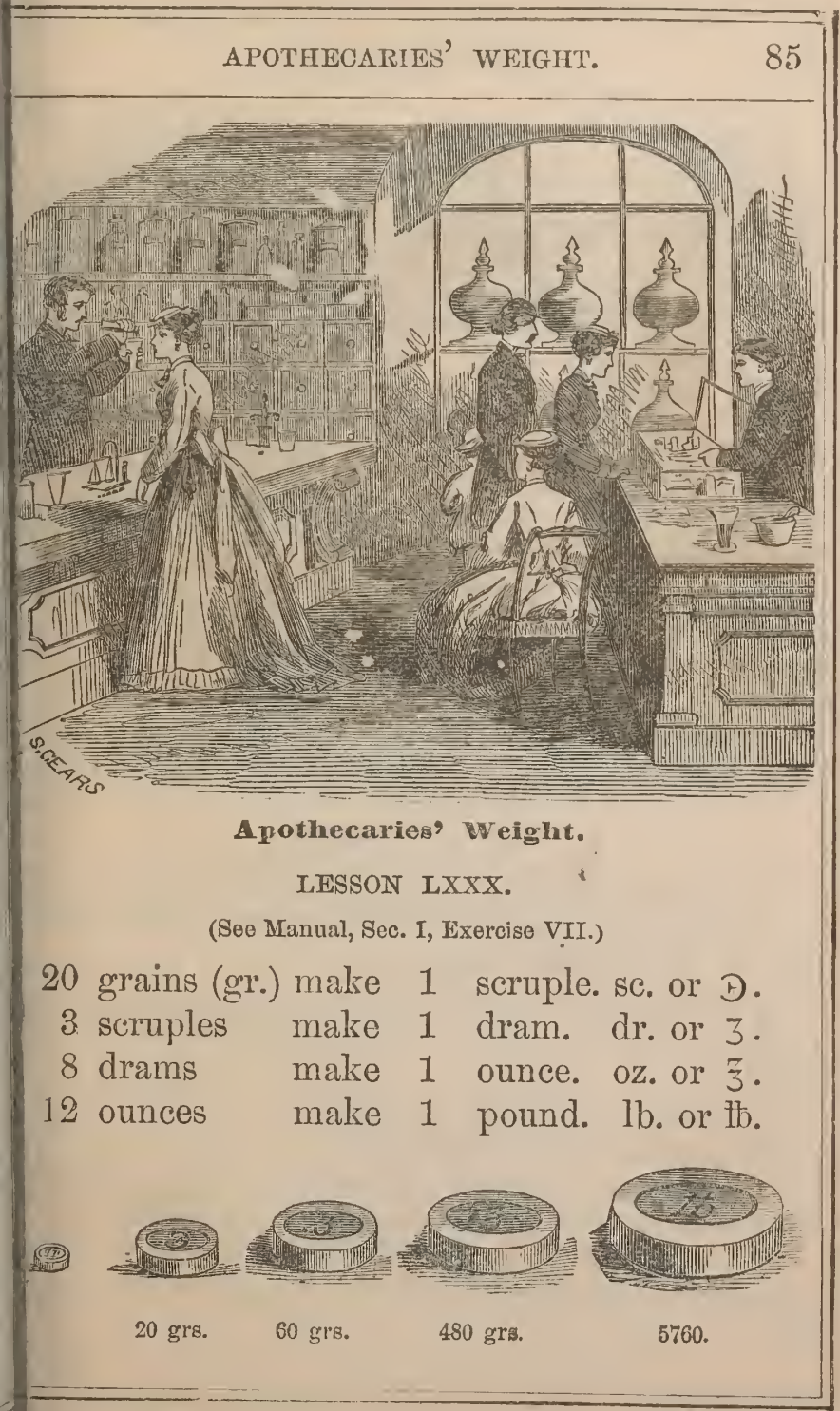




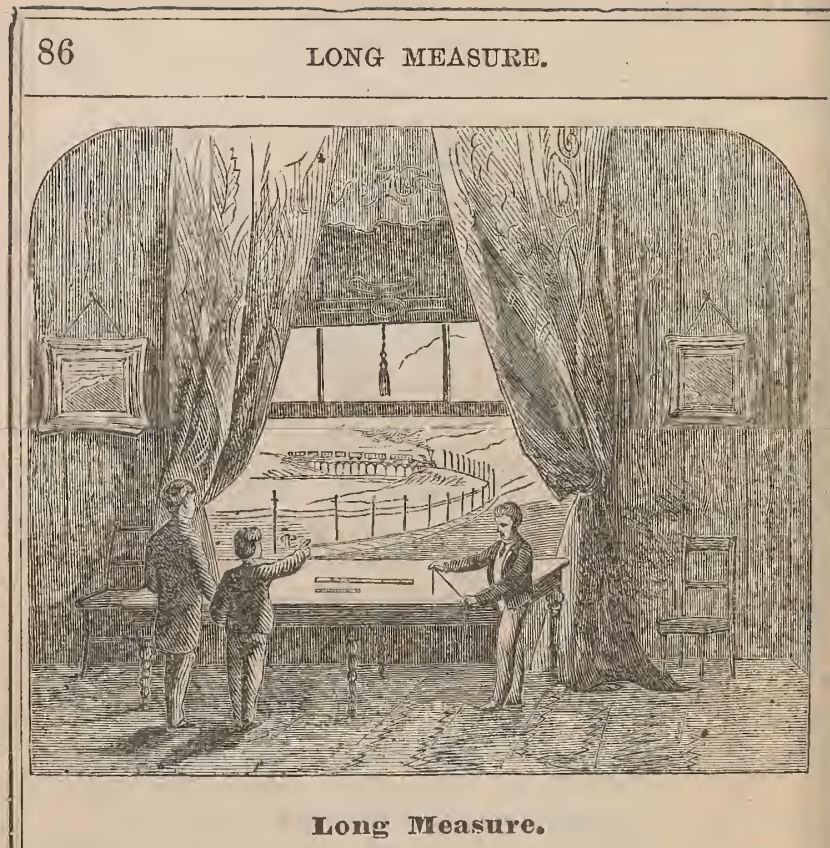

TEAOHER.-Arthur, can you tell me how far a mile is? ARTHCr.-If I place 12 sticks an inch long in a row, the row will be a foot long.

If I make a measure 3 times as long as the row of sticks, it will be a yard measure, like the one on the table.

Five and a half times the yard measure will be a rod, and is just the width of the room.

Forty times the width of the room is the distance between every other telegraph pole, or one furlong.

Eight times the distance between every other telegraph pole is the distance to the railroad-bridge, or one mile. 


\section{LESSON IXXXI.}

(See Manual, Sec. I., Exercise VII.)

\section{long Ifleasure.}

Long measure is used for measuring distance. TABLE.

12 inches (in.) make 1 foot. ft.

3 feet make 1 yard. yd.

$5 \frac{1}{2}$ yards

$\left.\begin{array}{l}5 \frac{1}{2} \text { yards } \\ 11 \text { half yards } \\ 40 \text { rods }\end{array}\right\}$ $1\left\{\begin{array}{c}\text { rod, perch, } \\ \text { or pole. }\end{array}\right\}$ rd. make 1 furlong. fur.

8 furlongs make 1 mile. mi. $69 \frac{1}{4}$ statute miles 277 quarter miles $\}$ make 1 degree. deg. 360 degrees make 1 circle of the earth. cir.

\section{Gunter's Chain Mi easure.}

Gunter's Chain Measure is used by surveyors. TABLE.

25 links (li.) make 1 rod. rd.

4 rods or 100 links $\}$ make 1 chain. ch. 80 chains make 1 mile. $\mathrm{mi}$.

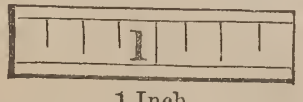

1 Inch.

Note.-A link is about $7 \frac{7}{8}$ inches in length. 


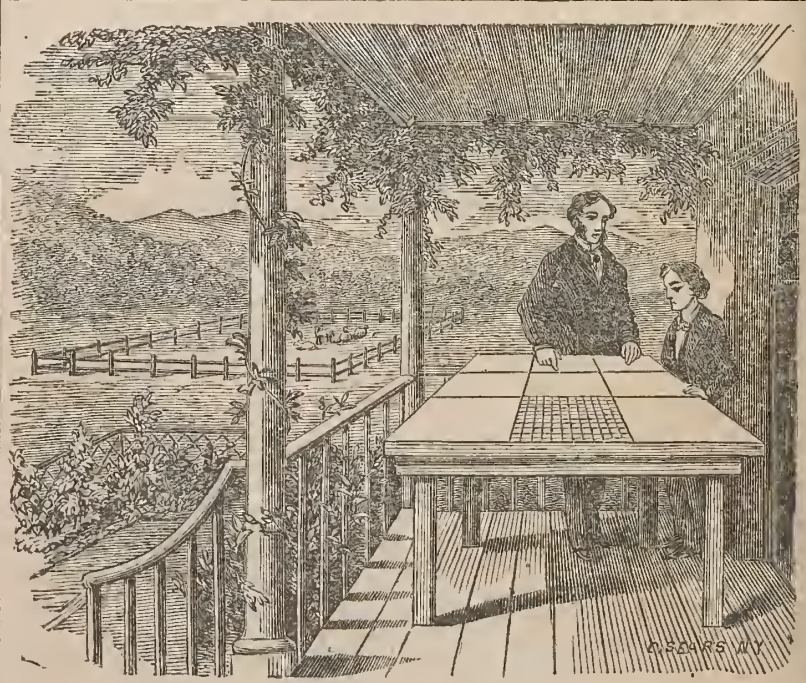

\section{Square Irieasure.}

Fatmer.-Charley, if you wish to know the size of an acre, cut 144 pieces of paper one inch square, and place them on the table; this is called a square foot.

To make a square yard, place 9 single squares in a square, which is just the size of the table.

To make a piece of land the size of the grass-plat in the door-yard, it will require $30 \frac{1}{x}$ square yards, which is called a square rod.

Forty square rods is just one rood, or a quarter of an acre of land, and is the size of the lotacross the road in which you see the sheep.

Four such lots contain 4 roods of land, and is called an acre. 


\section{IESSON LXXXII.}

(See Manual, Sec. I., Exercise VII.)

\section{Square Mreasure.}

Square measure is used in computing the area of surfaces.

TABLE.

144 square inches (in.) make 1 square foot.

9 square feet make 1 square yard.

$\left.\begin{array}{l}30 \frac{1}{4} \text { square yards or } \\ 121\end{array}\right\}$ make 1 square rod.

40 square rods make $1\{$ rood or quarter

$4\left\{\begin{array}{c}q u a r t e r \text { acres or } \\ \text { roods }\end{array}\right\}$ make 1 acre.

640 acres make $1\left\{\begin{array}{c}\text { square mile or } \\ \text { section. }\end{array}\right.$

\section{Surveyors' Square Mireasure.}

Surveyors' square measure is used in computing the area or contents of portions of land.

\section{TABLE.}

625 square links make 1 square rod. sq. rd. 16 square rods make 1 square chain. sq. ch. 10 square chains make 1 acre. A. 640 acres make 1 square' mile. sq. mi. 36 square miles make 1 township. T. 


\section{0 ' CUBIC MEASURE.}

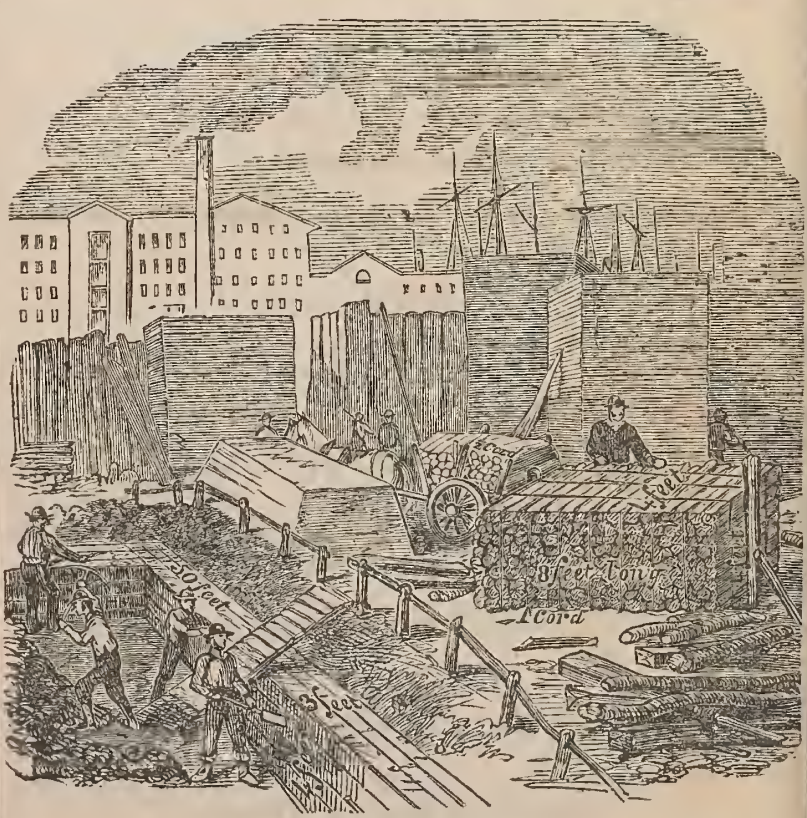

Cubic Mleasure.

In building the walls of a cellar, or of a building, the amount of stone or brick used is determined by culvic measure. $\Lambda$ cubic inch is a square block 1 inch long, 1 inch wide, and 1 inch thick, thus :

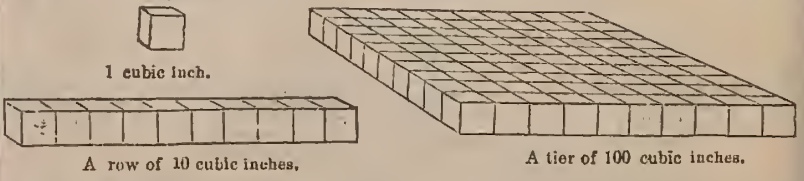




\section{IESSON LXXXII.}

(See Manual, Sec. I., Exercise VII.)

\section{Cubic Ireasure.}

Cubic measure is used to estimate the contents of solids:

TABLE.

1728 cubic inches

27 cubic feet

40 cubic ft. of round timber or

50 cubic ft. of hewn timber

16 cubic feet

8 cord feet or

128 cubic feet

$24 \frac{3}{4}$ cubic feet make 1 cubic foot. make 1 cubic yard.

make 1 ton or load. make 1 cord foot. make 1 cord of wood. make $1\left\{\begin{array}{l}\text { perch of } \\ \text { stone or } \\ \text { masonry. }\end{array}\right.$

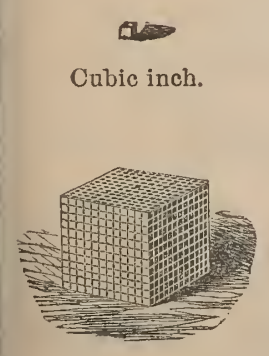

Cubic foot.

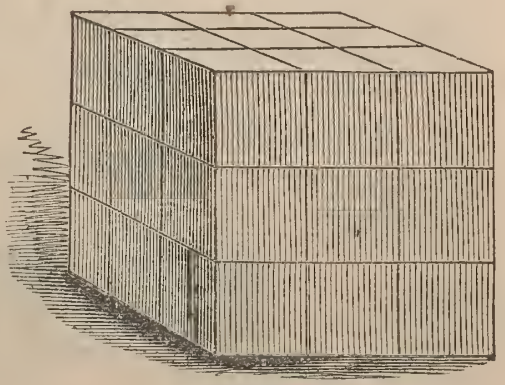

Cubic yard. 


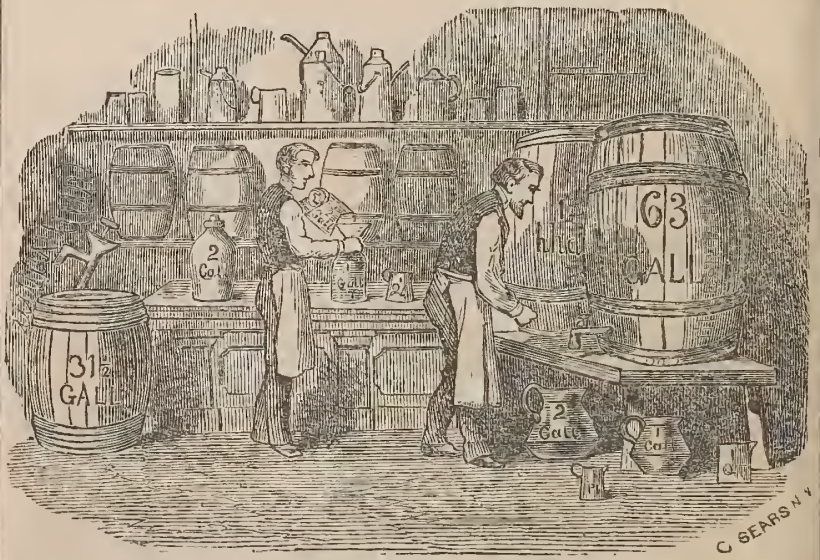

\section{Vine Minsure.}

Wine measure is used in measuring liquids.

\section{IJISSON XXXIX.}

4 gills

2 pints

4 quarts

$31 \frac{1}{2}$ yallons or

63 half gal.

2 barrels or

63 gallons make 1 pint.

make 1 quart.

make 1 gallon.

make 1 barrel.

make 1 hogshead. hhd. pt.

qt. gal.

bbl.
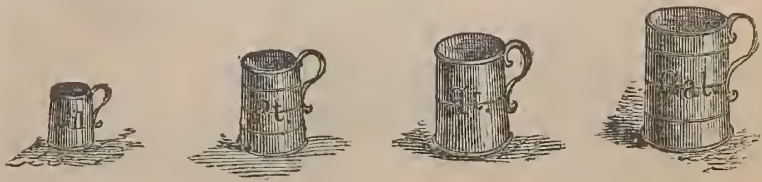


\section{4 \\ MIE $\triangle$ SURE OF TIMEE.}

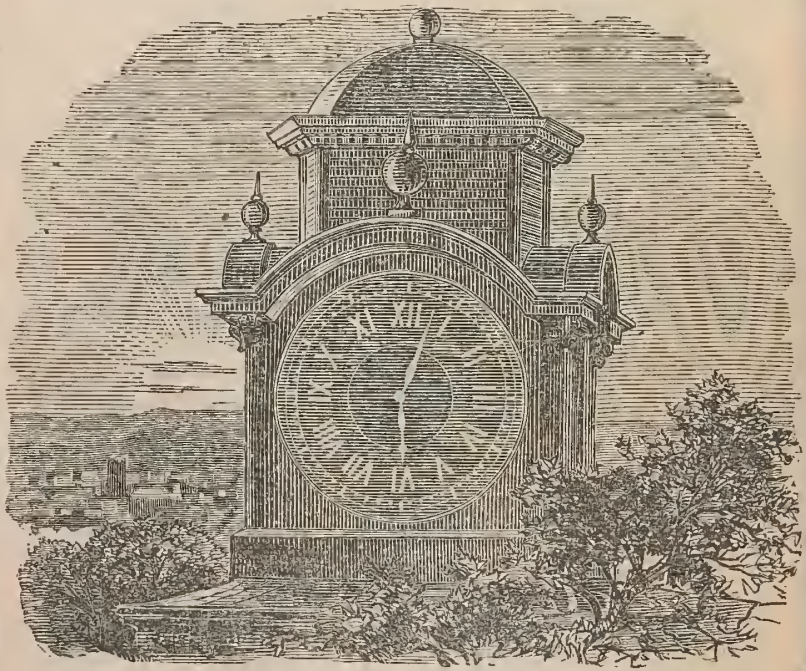

II

Time is the measure of duration.

LESSON LXXXVI.

(See Manual, Sec. I., Exercise V.)

60 seconds (sec.)

60 minutes

make 1 minute. min.

24 hours male 1 hour. h.

7 days make 1 day. da.

4 weeks and 2 days or $\}$ make one month. mo.

30 days

365 days

52 weeks

12 calendar months

make a year. - yr. make a year. yr. make a year. yr. 


\section{LES O N LXXXVII.}

(See Mantral, Sec. I., Exercise VI.)

\section{Circular IT casure.}

Circular measure is used in measuring ares of circles.

\section{TABBLE.}

60 seconds $\left({ }^{\prime \prime}\right)$ make 1 minute.

60 minutes

"6 1 degree.

90 degrees

66 1 quadrant.

qad.

4 quadrants

" 1 circumference. cir.

\section{Angular IIreasure.}

Angular measure is used for measuring difference of directions.

TABLE.

60 seconds (") make 1 minute.

60 minutes " 1 degree.

90 degrees " 1 right angle. r. a.

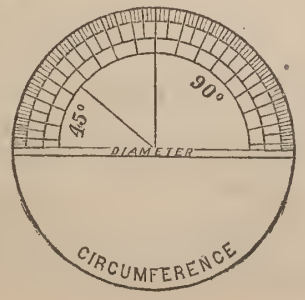




\section{IESSON IXXXVIII.}

\section{Firiscenlaneous 'rables. \\ DIVISION OF THE YEAR.}

Season. Names of months. No. of days. Abbreviations.

\begin{tabular}{|c|c|c|}
\hline Winter, & $\begin{array}{l}\text { 1. January, } \\
\text { 2. February, }\end{array}$ & $\begin{array}{l}31 \text { क } \\
28 \text { or } 29\end{array}$ \\
\hline Spring, & $\begin{array}{l}\text { 3. March, } \\
\text { 4. April, } \\
\text { 5. May, }\end{array}$ & $\begin{array}{l}31 \text {. } \\
30 \\
31\end{array}$ \\
\hline Summer, & $\begin{array}{l}\text { 6. June, } \\
\text { 7. July, } \\
\text { 8. August, }\end{array}$ & $\begin{array}{l}30 \\
31 \\
31\end{array}$ \\
\hline Autumn, & $\begin{array}{l}\text { 9. September, } \\
\text { 10. October, } \\
\text { 11. November, }\end{array}$ & $\begin{array}{r}-30 \\
31 \\
30\end{array}$ \\
\hline Winter, & 12. December, & $\frac{31}{365 \text { or } 366}$ \\
\hline
\end{tabular}

COUNTING.

12 units or things make 1 dozen.

12 dozen

12 gross

20 units

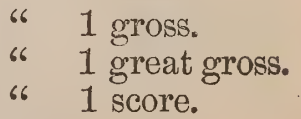

PAPER.

24 sheets make 1 quire. 20 quires " 1 ream.

2 reams " 1 bundle.

5 bundles " 1 bale. 


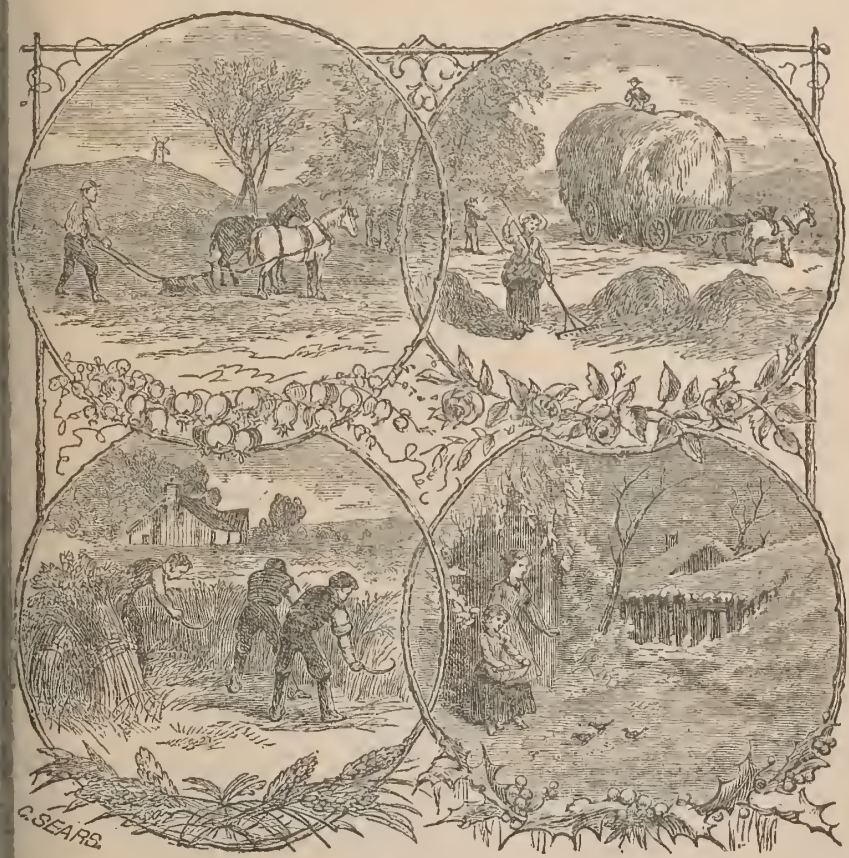

\section{Seasons.}

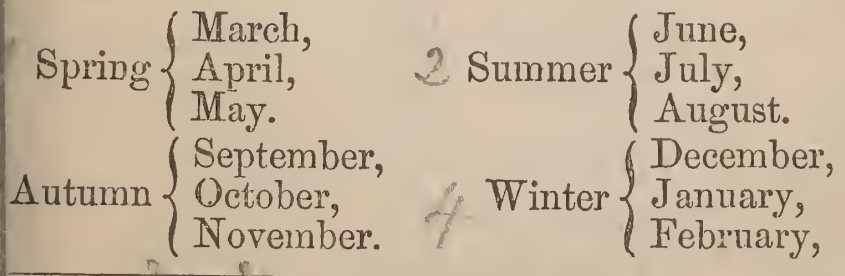

Thirty days hath September, April, June, and November,

February hath twenty-eight, * And thirty-one the others rate.

* Every leap-year, February has twenty-nine days. 
ROMAN NOTATION.

\section{LESSON LXXXIX.}

\section{Table of Fonnan Notation.}

I denotes one.

II " two.

III " three.

IV " four.

V " five.

VI " six.

VII " seven.

VIII " eight.

IX " nine.

$\mathrm{X}$ " ten.

XI " eleven.

XII " twelve.

XIII " thirteen.

XIV " fourteen.

$X V$ " fifteen.

XVI “ sixteen.

XVII “ seventeen.

XVIII “ eighteen.

XIX " nineteen.

$X X$ " twenty.

XXI “ twenty-one.

XXII " twenty-two.

XXII “ twenty-three.

XXIV " twenty-four.
XXX denotes thirty.

XL "forty.

L " fifty.

LX " sixty.

LXX "seventy.

LXXX " eighty.

$\mathrm{XC}$ "ninety.

C " one hundred.

CC "two hundred.

CCC "three hundred.

CCCC "four hundred.

$\mathrm{D}$ " five hundred.

DC " six hundred.

DCC "seven hundred. DCCC " eight hundred. DCCCC " nine hundred.

M "one thousand.

MD " fifteen hundred

MM "two thousand.

$\overline{\mathrm{X}}$ " ten thousand.

$\overline{\mathrm{XI}}$ "eleven th'sand.

$\overline{\mathrm{M}}$ "one million.

$\overline{\mathrm{MM}}$ "two millions. 




\section{FELTER'S ARITHMETICS. \\ CORCETETE COURSE.}

\section{FELTER'S PRIMARY ARITHMETIC.}

Mr. tur and Written Licercises Alternated. Elegantly Illustratea. FIIIER'S INIERMIDDIAE ARITHMETIC. Mental and Written Exercises.

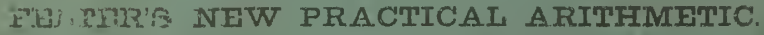
Mental and Written Exercises.

\section{Independent.}

FHLTER'S FIRST IESSONS IN NUMBERS.

Uial and Slate Exerciss. F'ully Illustrated.

Thrs series of books have been so long before the public, and have become so widely and farorably known, that little need be said toward pointing out their many peculiar features of excellence.

FELTER's SERIES initiated the plan, since imitated by different new series, alternating mental and writen exercises, throughout the body of their texts.

Teachers who have heretofore used Felter's smaller books with such emment satisfaction to themselves and their pupts will be aratifed to know that the series has been completed by the subsitution of an entirely new, higher book. called

\section{FELTER'S NEW FRACTICAL ARITEMETIC,} Der injig the best features of Felter's admirable systen, vil.f. Sxinr H. PEABODY, of the Massachusetts Agricultural Colle $c^{2}$ : $t$ Alulerst, anthor of "Elements of Istronomy."

Wherever used, Felter's admirable Text-books have been pronoumred by pracrical teachers to contain the most perfect system of arithmetical instruction published. The crading of the bonds is simpl, systematic and complete, and jeculiar to the scries. The works are the result of a long exp-rience and service in the he rom, and $j$ is belier ed they will meet the requirenuents of ar trade of schools. 